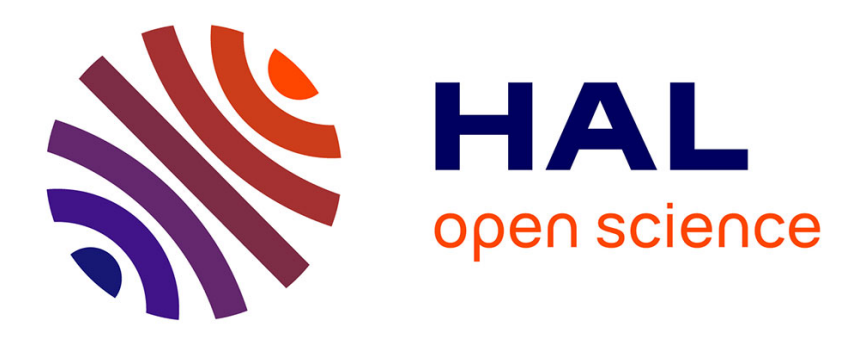

\title{
From Analogical Proportion to Logical Proportions
}

Henri Prade, Gilles Richard

\section{To cite this version:}

Henri Prade, Gilles Richard. From Analogical Proportion to Logical Proportions. Logica Universalis, 2013, vol. 7 ( $\left.\mathrm{n}^{\circ} 4\right)$, pp. 441-505. 10.1007/s11787-013-0089-6 . hal-01138469

\section{HAL Id: hal-01138469 \\ https://hal.science/hal-01138469}

Submitted on 2 Apr 2015

HAL is a multi-disciplinary open access archive for the deposit and dissemination of scientific research documents, whether they are published or not. The documents may come from teaching and research institutions in France or abroad, or from public or private research centers.
L'archive ouverte pluridisciplinaire HAL, est destinée au dépôt et à la diffusion de documents scientifiques de niveau recherche, publiés ou non, émanant des établissements d'enseignement et de recherche français ou étrangers, des laboratoires publics ou privés. 


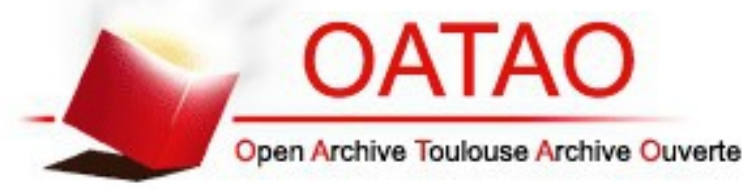

\section{Open Archive TOULOUSE Archive Ouverte (OATAO)}

OATAO is an open access repository that collects the work of Toulouse researchers and makes it freely available over the web where possible.

This is an author-deposited version published in : http://oatao.univ-toulouse.fr/ Eprints ID : 12864

To link to this article : DOI :10.1007/s11787-013-0089-6

URL : http://dx.doi.org/10.1007/s11787-013-0089-6

To cite this version : Prade, Henri and Richard, Gilles From Analogical Proportion to Logical Proportions. (2013) Logica Universalis, vol. 7 ( $\left.{ }^{\circ} 4\right)$. pp. 441-505. ISSN 1661-8297

Any correspondance concerning this service should be sent to the repository administrator: staff-oatao@,listes-diff.inp-toulouse.fr 


\title{
From analogical proportion to logical proportions
}

\author{
Henri Prade and Gilles Richard
}

\begin{abstract}
Given a 4-tuple of Boolean variables $(a, b, c, d)$, logical proportions are modeled by a pair of equivalences relating similarity indicators $(a \wedge b$ and $\bar{a} \wedge \bar{b}$ ), or dissimilarity indicators $(a \wedge \bar{b}$ and $\bar{a} \wedge b)$ pertaining to the pair $(a, b)$, to the ones associated with the pair $(c, d)$. There are 120 semantically distinct logical proportions. One of them models the analogical proportion which corresponds to a statement of the form " $a$ is to $b$ as $c$ is to $d$ ". The paper inventories the whole set of logical proportions by dividing it into 5 subfamilies according to what they express, and then identifies the proportions that satisfy noticeable properties such as full identity (the pair of equivalences defining the proportion hold as true for the 4-tuple $(a, a, a, a))$, symmetry (if the proportion holds for $(a, b, c, d)$, it also holds for $(c, d, a, b))$, or code independency (if the proportion holds for $(a, b, c, d)$, it also holds for their negations $(\bar{a}, \bar{b}, \bar{c}, \bar{d}))$. It appears that only 4 proportions (including analogical proportion) are homogeneous in the sense that they use only one type of indicator (either similarity or dissimilarity) in their definition. Due to their specific patterns, they have a particular cognitive appeal, and as such are studied in greater details. Finally, the paper provides a discussion of the other existing works on analogical proportions.
\end{abstract}

Keywords. proportion, analogical proportion, propositional logic, similarity, dissimilarity, conditional object, square of opposition, Blanché hexagon, $\mathrm{Pi}$ aget group.

\section{Introduction}

Proportions, understood as the identity of relations between two ordered pairs of entities, say $(A, B)$ and $(C, D)$ play a crucial role in the way the human mind 
perceives the world and tries to make sense of it. Thus, proportions involve four terms, which may not be all distinct.

In mathematics, a proportion is a statement of equality between the result of operations involving numerical quantities (i.e., $A, B, C, D$ are numbers). The geometric proportion amounts to state the equality of two ratios, i.e., $A / B=C / D$, while the arithmetic proportion compares two pairs of numbers in terms of their differences, i.e., $A-B=C-D$. In these equalities, which emphasize the symmetric role of the pairs $(A, B)$ and $(C, D)$, geometric or arithmetic ratios have an implicit comparative flavor, and the proportions express the invariance of the ratios. Note that by cross-product for geometric proportion, or by addition for the arithmetic one, the two proportions are respectively equivalent to $A D=B C$ and to $A+D=$ $B+C$, which makes clear that $B$ and $C$, or $A$ and $D$, can be permuted without changing the validity of the proportion. Moreover, mathematical proportions are at the basis of reasoning procedures that enable us to "extrapolate" the fourth value knowing three of the four quantities. Indeed, assuming that $D$ is unknown, one can deduce $D=C \times B / A$ in the first case, which corresponds to the wellknown "rule of three", or $D=C+(B-A)$ in the second case. Besides, continuous proportions where $B=C$ are directly related to the idea of averaging, since taking $B=C$ as the unknown respectively yields the geometric mean $(A D)^{1 / 2}$ and the arithmetic mean $(A+D) / 2 .^{1}$ Thus, mathematical proportions have the following distinctive features:

1. they involve 4 terms;

2. they satisfy an identity property, i.e., they trivially hold for $(A, B)=(C, D)$;

3. they are symmetric;

4. central terms, or extreme terms, can be permuted;

5. 3 of the terms in a proportion uniquely determine the 4 th one;

6. in a proportion, 2 terms are in mean positions with respect to the 2 others, namely $(B, C)$ w.r.t. $(A, D)$ (as well as $(A, D)$ w.r.t. $(B, C)$, due to $(3))$.

Proportions have an important place in Ancient Greek mathematics, and play a role in other areas as well (think for instance of the golden ratio obtained as the solution $\varphi=A / B$ of the geometric proportion $(A+B) / A=A / B)$. Thus, in the Book 5 about Justice of his Nicomachean Ethics, Aristotle makes explicit reference to geometric proportions when discussing what is "fair":

The just, then, is a species of the proportionate (proportion being not a property only of the kind of number which consists of abstract units, but of number in general). For proportion is equality

\footnotetext{
${ }^{1} \mathrm{~A}$ third type of proportion, called harmonic, combines geometric and arithmetic comparisons by stating that $A / D=(A-B) /(C-D)$. It is equivalent to $2 A D=A C+B D$, which expresses that the double product of the extreme terms is the sum of the product of the odd rank terms and of the product of the even rank terms. In this proportion, the pairs $(A, B)$ and $(C, D)$ no longer play a symmetric role, as they do for the geometric and arithmetic proportions. When taking $B=C$ as the unknown number, one obtains the harmonic mean $2 A D /(A+D)$. The arithmetic, geometric and harmonic means are known as the Pythagorean means.
} 
of ratios, and involves four terms at least. [...] The ratio between one pair is the same as that between the other pair; for there is a similar distinction between the persons and between the things. As the term $A$, then, is to $B$, so will $C$ be to $D$, and therefore, alternando, as $A$ is to $C, B$ will be to $D$. Therefore also the whole $[A+C]$ is in the same ratio to the whole $[B+D]$; and this coupling the distribution effects, and, if the terms are so combined, effects justly. The conjunction, then, of the term $A$ with $C$ and of $B$ with $D$ is what is just in distribution, and this species of the just is intermediate, and the unjust is what violates the proportion; for the proportional is intermediate, and the just is proportional.

(translation by W. D. Ross)

But Aristotle does not only make use of quantitative proportions. In many places, he also considers comparative relations between 4 terms that form in modern words an analogical proportion, again expressing an identity of relation between two ordered pairs. For instance, in the Book 1 (Part 17) of his Topics, when discussing the idea of "likeness", he provides examples of such proportions between things belonging to different genera:

$[\cdots]$ As sight is in the eye, so is reason in the soul, and as is a calm in the sea, so is windlessness in the air. Practice is more especially needed in regard to terms that are far apart; for in the case of the rest, we shall be more easily able to see in one glance the points of likeness. We should also look at things which belong to the same genus, to see if any identical attribute belongs to them all, e.g. to a man and a horse and a dog; for in so far as they have any identical attribute, in so far they are alike.

(translation by W. A. Pickard-Cambridge)

Another example by Aristotle can be found in his Prior Analytics:

For example let $A$ be evil, $B$ making war against neighbours, $C$ Athenians against Thebans, D Thebans against Phocians. If then we wish to prove that to fight with the Thebans is an evil, we must assume that to fight against neighbours is an evil. Evidence of this is obtained from similar cases, e.g. that the war against the Phocians was an evil to the Thebans. Since then to fight against neighbours is an evil, and to fight against the Thebans is to fight against neighbours, it is clear that to fight against the Thebans is an evil. Now it is clear that $B$ belongs to $C$ and to $D$ (for both are cases of making war upon one's neighbours) and that $A$ belongs to $D$ (for the war against the Phocians did not turn out well for the Thebans): but that $A$ belongs to $B$ will be proved through $D$. Similarly if the belief in the relation of the middle term to the extreme should be produced by several similar cases. Clearly then to argue by example is neither like reasoning from part to whole, 
nor like reasoning from whole to part, but rather reasoning from part to part, when both particulars are subordinate to the same term, and one of them is known.

(translation by A. J. Jenkinson)

Thus, due to their structural similarity with mathematical proportions, statements of the form " $A$ is to $B$ as $C$ is to $D$ " have been called analogical proportions, where $A, B, C, D$ are no longer necessarily numbers, but may refer to situations described through words, equations, pictures, ... and they can be used for the so called "analogical reasoning". Since Aristotle's time, analogical reasoning has received a lot of attention from researchers in many areas, and more particular from scholars in philosophy, anthropology, cognitive psychology and linguistics (see, e.g., $[17,32,47,21,28,26,27,34,25,18,46])$, including artificial intelligence more recently [31]. However, strangely enough, it seems that there has been no attempt at providing some logical model of analogical proportions up to two noticeable exceptions, which have been however fully ignored by the mainstream literature.

The first exception can be found in the Annex of a 1952 French book by the psychologist Jean Piaget [57] (see also [58] pp. 35-37), where the following definition of a so-called proportion logique is given: 4 propositions $A, B, C$, and $D$ make a logical proportion if the two following conditions hold $A \wedge D=B \wedge C$ and $A \vee D=B \vee C$. However, this logical proportion, which turns out, as we shall see, to be one among the possible (equivalent) definitions of an analogical proportion, usually denoted $A: B:: C: D^{2}$, is introduced by Piaget without any reference to analogy ${ }^{3}$.

The second exception is provided by Sheldon Klein [36, 37, 38], a computer scientist with a strong background in anthropology and linguistics, who introduced a so-called ATO operator (where ATO stands for "Appositional Transformation Operator"). This Boolean operator, which is based on the logical equivalence connective, amounts to compute the 4th argument of an analogical proportion between Boolean vectors (describing the items in terms of binary features) by applying $D=C \equiv(A \equiv B)$ componentwise. Strictly speaking, this calculation does not always fit with the correct definition of an analogical proportion, as we shall see.

In this paper we provide a systematic investigation of logical proportions viewed in terms of 2 equivalences between conjunctions of pairs of atoms which may be negated or not. The conjunction of two positive or two negative atoms, by modeling their common truth or common falsity, expresses similarity, while the conjunction of a positive atom and a negative atom expresses dissimilarity.

\footnotetext{
${ }^{2}$ The use of "::" for denoting the equality of ratios in (numerical) proportions (see [73] p. 394) dates back to the 17th century mathematician William Oughtred [10], while ":" just denotes ratios. Interestingly enough, this notation has been currently used for a longtime to denote analogical proportions, although they are generally non numerical, while it is no longer in use for numerical proportions.

${ }^{3}$ However, in a previous book [55] (pp. 97-99), Piaget informally investigates a similar idea, even using an example of linguistic analogical proportion, still without explicitly mentioning analogy.
} 
The analogical proportion then appears as a particular logical proportion, still especially remarkable.

The paper is organized as follows. The next section 2 introduces a general notion of logical proportions as pairs of logical equivalences linking 4 Boolean variables $a, b, c, d$ combined two by two conjunctively, possibly in a negated form. It also provides a typology of logical proportions into five classes. Section 3 studies the semantics of logical proportions in terms of truth tables, and highlights the fact that any logical proportion is true for 6 and only 6 valuations among $2^{4}=16$ possible ones. Section 4 investigates meaningful properties of logical proportions, such as identity, symmetry, permutability, code independency, transitivity, equation solvability by identifying the proportions that satisfy them among the 120 distinct logical proportions. Section 5 further studies the class of 4 homogeneous logical proportions which includes analogical proportion, and analyzes them at the light of the square of oppositions and other more general geometrical constructs. Section 6 provides a structured overview of related works, first coming back on the two early attempts by Piaget and Klein at providing a logical view of (ana)logical proportions, before reviewing other logical and non logical approaches to analogical reasoning.

\section{Logical proportions}

Before introducing the formal definitions, let us briefly clarify the notations used.

- When dealing with Boolean logic, $a, b, \ldots$ denote propositional variables (having 0 or 1 as truth value), and we use the standard symbols $\wedge, \vee$ to build up formulas (with parenthesis when needed). For the negation operator, instead of using the standard $\neg$ symbol, we will use $\bar{a}$ to denote $\neg a$. This is done for saving space when writing long formulas. As usual $T$ (resp. $\perp$ ) denotes the always true (resp. false) proposition.

- 0 and 1 denote the Boolean truth values, and a valuation $v$ is just a function from the set of propositional variables to $\{0,1\}$. As we generally consider only 4 variables $a, b, c, d$, it is sometimes convenient to refer to $v$ by the 4 -tuple $(v(a), v(b), v(c), v(d))$.

- When we propose a new definition, we will use the symbol $\triangleq$ meaning definitional equality. For instance, $F(a, b, c, d) \triangleq a \vee b \vee c \vee d$. The right hand side of the equation is the definition of the left-hand side.

- When we consider syntactic identity, we use $=_{I d}$ : for instance $a \wedge b={ }_{I d} a \wedge b$ but we do not have $a \wedge b={ }_{I d} b \wedge a$.

- Finally, the symbol $\equiv$ is reserved for the equivalence, i.e.

$$
\begin{gathered}
a \rightarrow b \triangleq \bar{a} \vee b \\
a \equiv b \triangleq a \rightarrow b \wedge b \rightarrow a
\end{gathered}
$$




\subsection{Similarity and dissimilarity indicators}

Generally speaking, the comparison of two items $A$ and $B$ relies on the representation of these items. For instance, the items may be represented as a set of features $\mathcal{A}$ and $\mathcal{B}$. Then, one may define a similarity measure. This is the aim of the wellknown work of Amos Tversky [80], taking into account the common features, the specificities of $A$ w.r.t. $B$, and the specificities of $B$ w.r.t. $A$, respectively modeled by $\mathcal{A} \cap \mathcal{B}, \mathcal{A} \backslash \mathcal{B}$, and $\mathcal{B} \backslash \mathcal{A}$. Here, we are not looking for any global measure of similarity, we are rather interested in keeping track in what respect items are similar and in what respect they are dissimilar using Boolean indicators. This is why we adopt a logical setting: features are viewed as Boolean properties. Let $P$ be such a property, which can be seen as a predicate: $P(A)$ may be true (in that case $\neg P(A)$ is false), or false. When comparing two items $A$ and $B$ w.r.t. such a property $P$, it makes sense to consider $A$ and $B$ similar:

- when $P(A) \wedge P(B)$ is true or

- when $\neg P(A) \wedge \neg P(B)$ is true.

In the remaining cases:

- when $\neg P(A) \wedge P(B)$ is true or

- when $P(A) \wedge \neg P(B)$ is true,

we can consider $A$ and $B$ as dissimilar w.r.t. property $P$. Since $P(A)$ and $P(B)$ are ground formulas, they can simply be considered as Boolean variables, and denoted $a$ and $b$ by abstracting w.r.t. $P$. If the conjunction $a \wedge b$ is true, the property is satisfied by both items $A$ and $B$, while the property is satisfied by neither $A$ nor $B$ if $\bar{a} \wedge \bar{b}$ is true. The property is true for $A$ only (resp. $B$ only) if $a \wedge \bar{b}$ (resp. $\bar{a} \wedge b$ ) is true. This is why we call such a conjunction of Boolean literals an indicator, and for a given pair of Boolean variables $(a, b)$, we have exactly 4 distinct indicators:

- $a \wedge b$ and $\bar{a} \wedge \bar{b}$ that we call similarity indicators,

- $a \wedge \bar{b}$ and $\bar{a} \wedge b$ that we call dissimilarity indicators.

Let us observe that negating anyone of the two terms of a dissimilarity indicator turns it into a similarity indicator, and conversely. Hence, negating the two terms of an indicator yields an indicator of the same type. But negating an indicator does not give an indicator. Besides, it is worth noting that the indicators we are going to use are also those involved in Tversky's similarity measure. Namely, his similarity measure $s(A, B)$ between two sets of features $A$ and $B$ is a combination $F$ involving the 3 components $A \cap B, A \backslash B$ and $B \backslash A$, i.e. $s(A, B)=F(A \backslash B, B \backslash A, A \cap B)$, where $\backslash$ denotes set difference.

\subsection{Defining logical proportions}

In a logical proportion, 4 items are involved: $A, B, C$, and $D$. Let $a, b, c$ and $d$ the 4 Boolean variables corresponding to the same property. We have again 4 indicators per pair of Boolean variables, and a comparison of two pairs of items can be only based on these indicators. The simplest way for expressing a comparison is an equivalence between 2 indicators, like for instance $a \wedge \bar{b} \equiv c \wedge d$. 
A point has to be raised now: it seems natural that the informal idea of a logical proportion should be independent of the way we encode items in terms of the truth or the falsity of properties (just as a numerical proportion holds independently of the base used for encoding numbers, or of the system of units representing the quantities at hand). It means that the formula defining the proportion should be valid when we switch 0 to 1 and 1 to 0 . So let us consider a formula based on a unique equivalence between indicators, denoted $l_{1} \wedge l_{2} \equiv l_{3} \wedge l_{4}$, where the $l_{i}$ 's are literals. Let us now consider a valuation $v$ such that $v\left(l_{1}\right)=v\left(l_{2}\right)=v\left(l_{3}\right)=0$ and $v\left(l_{4}\right)=1$. Obviously this valuation makes the equivalence valid since $v\left(l_{1} \wedge l_{2}\right)=$ $v\left(l_{3} \wedge l_{4}\right)=0$. But when we switch 0 to 1 and 1 to 0 , it appears that the new valuation $v^{\prime}$ such that $v^{\prime}\left(l_{1}\right)=v^{\prime}\left(l_{2}\right)=v^{\prime}\left(l_{3}\right)=1$ and $v^{\prime}\left(l_{4}\right)=0$ does not validate the equivalence anymore. Then one equivalence is not enough if we are interested in "code-independency". We have to consider at least 2 equivalences to capture this behavior. For instance, $(a \wedge b \equiv c \wedge d) \wedge(\bar{a} \wedge \bar{b} \equiv \bar{c} \wedge \bar{d})$ clearly satisfies code independency.

As a consequence, it is legitimate to consider conjunctions of 2 equivalences between indicators, and such a conjunction will be called logical proportion [62, 64]. More formally, let us denote $I_{(a, b)}$ and $I_{(a, b)}^{\prime}$, (resp. $I_{(c, d)}$ and $\left.I_{(c, d)}^{\prime}\right) 2$ indicators for $(a, b)$ (resp. $(c, d))$. Note that $I_{(a, b)}$ (or $\left.I_{(a, b)}^{\prime}\right)$ refers to one element in the set $\{a \wedge b, \bar{a} \wedge b, a \wedge \bar{b}, \bar{a} \wedge \bar{b}\}$, and should not be considered as a functional symbol. Still, we use this notation for the sake of readibility. Then

Definition 1. A logical proportion $T(a, b, c, d)$ is the conjunction of 2 distinct equivalences between indicators of the form

$$
I_{(a, b)} \equiv I_{(c, d)} \wedge I_{(a, b)}^{\prime} \equiv I_{(c, d)}^{\prime}
$$

An example of such proportion is $((\bar{a} \wedge \bar{b}) \equiv(c \wedge \bar{d})) \wedge((\bar{a} \wedge b) \equiv(\bar{c} \wedge d))$ where

- $I_{(a, b)} \triangleq \bar{a} \wedge \bar{b}, I_{(c, d)} \triangleq c \wedge \bar{d}$,

- $I_{(a, b)}^{\prime} \triangleq \bar{a} \wedge b, I_{(c, d)}^{\prime} \triangleq \bar{c} \wedge d$.

Obviously, this formal definition goes beyond what may be expected from the informal idea of "logical proportion", since equivalences may be put between things that are not homogeneous (i.e., mixing similarity and dissimilarity indicators in various ways).

Let us first determine the number of logical proportions. Since we have to choose 2 distinct indicators among 4 for a given pair of variables $(a, b)$, we have $\left[\begin{array}{l}4 \\ 2\end{array}\right]=6$ candidate sets for $\left(I_{(a, b)}, I_{(a, b)}^{\prime}\right)$. Building the pair of equivalences then leads to $6 \times 6 \times 2=72$ logical proportions. Indeed there are $6 \times 6$ candidate sets for choosing a 4-tuple $\left(I_{(a, b)}, I_{(a, b)}^{\prime}, I_{(c, d)}, I_{(c, d)}^{\prime}\right)$, and there are 2 ways for combining these indicators according to Definition 1 . In this count, we have assumed that the 2 indicators that we choose for a pair are distinct, which is quite natural. It means that we use exactly 4 distinct indicators to build up a proportion: 2 for $(a, b)$ and 2 for $(c, d)$. But if we accept that the same indicator appears twice, i.e., we use 
only 3 indicators to build up a proportion (indeed using only 2 indicators would not allow to build up two distinct equivalences). Then on top of the 72 previous proportions, we have to add the $4 \times 6=24$ proportions that we can build using only one indicator for $(a, b)$ (and we have 4 choices), and 2 distinct ones for $(c, d)$ (for instance $(a \wedge \bar{b} \equiv c \wedge d) \wedge(a \wedge \bar{b} \equiv \bar{c} \wedge d)$ and similarly 24 when using one indicator only for $(c, d)$. So a total of 48 new proportions can be added. These proportions that use 3 indicators only are called "degenerated logical proportions"[62]. This leads to a total of $72+48=120$ logical proportions, included the degenerated ones, which are potentially semantically distinct.

Clearly, thanks to the De Morgan's laws, an equality $\bar{a} \wedge \bar{b}=\bar{c} \wedge \bar{d}$ is equivalent to $a \vee b=c \vee d$, without any use of a negation operator. Still, we stick for the moment to the use of conjunctions and negations on each side of the equivalences, which leaves the indicators explicit. The next subsection is devoted to a brief typology of all these proportions.

\subsection{Typology of logical proportions}

Depending on the way the indicators are chosen, one may mix the similarity and the dissimilarity indicators differently in the definition of a proportion. This leads us to distinguish a specific subfamily of proportions, the so-called degenerated proportions: those ones involving only 3 distinct indicators in their definition. For instance $(a \wedge b \equiv \bar{c} \wedge d) \wedge(\bar{a} \wedge b \equiv \bar{c} \wedge d)$ is such a proportion where $I_{(c, d)}={ }_{I d} I_{(c, d)}^{\prime}$.

For the remaining proportions, it is required that all the indicators appearing in the definition of the proportion are distinct. At this stage, it makes sense to distinguish between two types of indicators: similarity indicators that are denoted by $S$, and dissimilarity indicators that are denoted by $D$ : e.g., $D_{(a, b)} \in\{a \wedge \bar{b}, \bar{a} \wedge b\}$. With this notation, among the non-degenerated proportions, we can identify 4 subfamilies that we describe below.

2.3.1. The 4 homogeneous proportions. For these proportions, we do not mix different types of indicators in the 2 equivalences. The homogeneous proportions are of the form

or

$$
S_{(a, b)} \equiv S_{(c, d)} \wedge S_{(a, b)}^{\prime} \equiv S_{(c, d)}^{\prime}
$$

$$
D_{(a, b)} \equiv D_{(c, d)} \wedge D_{(a, b)}^{\prime} \equiv D_{(c, d)}^{\prime}
$$

Thus, it appears that only 4 proportions among 120 are homogeneous. They are (with names that will be explained later):

- analogy: $A(a, b, c, d)$, defined by

$$
((a \wedge \bar{b}) \equiv(c \wedge \bar{d})) \wedge((\bar{a} \wedge b) \equiv(\bar{c} \wedge d))
$$

- reverse analogy: $R(a, b, c, d)$, defined by

$$
((a \wedge \bar{b}) \equiv(\bar{c} \wedge d)) \wedge((\bar{a} \wedge b) \equiv(c \wedge \bar{d}))
$$


- paralogy: $P(a, b, c, d)$, defined by

$$
((a \wedge b) \equiv(c \wedge d)) \wedge((\bar{a} \wedge \bar{b}) \equiv(\bar{c} \wedge \bar{d}))
$$

- inverse paralogy: $I(a, b, c, d)$, defined by

$$
((a \wedge b) \equiv(\bar{c} \wedge \bar{d})) \wedge((\bar{a} \wedge \bar{b}) \equiv(c \wedge d))
$$

These 4 proportions are studied in details in Section 5. Analogy already appeared under this form in [52]; paralogy and reverse analogy were first introduced in [60], and inverse paralogy in [64]. While the analogical proportion (analogy, for short) reads " $a$ is to $b$ as $c$ is to $d$ " and expresses that " $a$ differs from $b$ as $c$ differs from $d$, and conversely $b$ differs from $a$ as $d$ differs from $c$ ", reverse analogy expresses that " $a$ differs from $b$ as $d$ differs from $c$, and conversely", paralogy expresses that "what $a$ and $b$ have in common, $c$ and $d$ have it also" 4 . Finally, inverse paralogy expresses that "what $a$ and $b$ have in common, $c$ and $d$ miss it, and conversely". As can be seen, inverse paralogy expresses a form of antinomy between pair $(a, b)$ and pair $(c, d)$. Note that we use two different words, "inverse" and "reverse", since the changes between analogy and reverse analogy on the one hand, and paralogy and inverse paralogy on the other hand, are not of the same nature. The meanings of the four above proportions is perhaps still more easy to grasp when moving from Boolean variables, to situations described in terms of sets of properties.

From now on, we denote analogy with $A$, paralogy with $P$, reverse analogy with $R$, inverse analogy with $I$. When we need to denote any unspecified proportion, we will use the letter $T$.

2.3.2. The 16 conditional proportions. Their expression is made of the conjunction of an equivalence between similarity indicators and of an equivalence between dissimilarity indicators. Thus, they are of the form

$$
S_{(a, b)} \equiv S_{(c, d)} \wedge D_{(a, b)} \equiv D_{(c, d)}
$$

There are 16 conditional proportions $(2 \times 2$ choices per equivalence). They appear in Table 9 in Annex A. An example is

$$
((a \wedge b) \equiv(c \wedge d)) \wedge((a \wedge \bar{b}) \equiv(c \wedge \bar{d}))
$$

Let us explain the term "conditional". It comes from the fact that these proportions express "equivalences" between conditional statements. Indeed, it has been advocated in [19] that a rule "if $a$ then $b$ " can be seen as a three valued entity that is called "conditional object" and denoted $b \mid a[16]$. This entity is:

- true if $a \wedge b$ is true. The elements making it true are the examples of the rule "if $a$ then $b$ ",

- false if $a \wedge \bar{b}$ is true. The elements making it true are the counter-examples of the rule "if $a$ then $b$ ",

\footnotetext{
${ }^{4}$ Although we have been using the term "paralogy" since we introduced this proportion in [60], "parallelogy" could be a more accurate term for expressing a logic of parallelism between situations $(a, b)$ and $(c, d)$.
} 
- undefined if $\bar{a}$ is true. The rule "if $a$ then $b$ " is then not applicable.

Thus, the above proportion $((a \wedge b) \equiv(c \wedge d)) \wedge((a \wedge \bar{b}) \equiv(c \wedge \bar{d}))$ may be denoted $b|a:: d| c$ combining the two conditional objects in the spirit of the usual notation for analogical proportion. Indeed, it expresses a semantical equivalence between the 2 rules "if $a$ then $b$ " and "if $c$ then $d$ " by stating that they have the same examples, i.e. $(a \wedge b) \equiv(c \wedge d))$ and the same counter-examples $(a \wedge \bar{b}) \equiv(c \wedge \bar{d})$.

It is worth noticing that such proportions have equivalent forms, e.g.:

$$
b|a:: d| c \equiv \bar{b}|a:: \bar{d}| c
$$

which agrees with the above semantics and more generally with the idea of conditioning. Indeed the examples "if $a$ then $b$ " are the counter-examples of "if $a$ then $\bar{b}$ ", and vice-versa. Due to this remark, it is enough to consider the equivalences between one of the 4 conditional objects $a|b, b| a, a|\bar{b}, b| \bar{a}$, and the 4 other conditional objects built with $(c, d)$, yielding $4 \times 4$ proportions as expected. Besides, 8 conditional proportions have been already considered in [64], but not the 8 remaining ones, since they do not satisfy the "full identity" property, as we shall see in the next section.

2.3.3. The 20 hybrid proportions. They are characterized by equivalences between similarity and dissimilarity indicators in their definitions. They are of the form

or

$$
S_{(a, b)} \equiv D_{(c, d)} \wedge S_{(a, b)}^{\prime} \equiv D_{(c, d)}^{\prime}
$$

$$
D_{(a, b)} \equiv S_{(c, d)} \wedge D_{(a, b)}^{\prime} \equiv S_{(c, d)}^{\prime}
$$

or

$$
S_{(a, b)} \equiv D_{(c, d)} \wedge D_{(a, b)} \equiv S_{(c, d)}
$$

They appear in Table 10 in Annex A. There are 20 hybrid proportions: 2 of the first kind, 2 of the second kind, 16 of the third kind since we have here 4 choices for an equivalence $S_{(a, b)} \equiv D_{(c, d)}$, and 4 choices for $D_{(a, b)} \equiv S_{(c, d)}$.

If we remember that negating anyone of the two terms of a dissimilarity indicator turns it into a similarity indicator, and conversely, we understand that changing $a$ into $\bar{a}$ (and $\bar{a}$ into $a$ ), or applying a similar transformation with respect to $b, c$, or $d$, turns

- an hybrid proportion into an homogeneous or a conditional proportion;

- an homogeneous or a conditional proportion into an hybrid proportion.

This indicates the close relationship of hybrid proportions with homogeneous and conditional proportions. More precisely,

- on the one hand there are 4 hybrid proportions such that replacing $a$ with $\bar{a}$ leads to the 4 homogeneous proportions $A, R, P, I$. They are obtained by the two first kinds of patterns for building hybrid proportions. Moreover, we shall see in the next section that they constitute with the 4 homogeneous proportions the 8 proportions that are the only ones satisfying "code independency" property.

- on the other hand, there are 16 remaining hybrid proportions, obtained by the third kind of pattern for building them. They can be written as the equivalence 
of 2 conditional objects, although they do not obey the conditional proportion pattern. For instance, $((\bar{a} \wedge b) \equiv(c \wedge d)) \wedge((a \wedge b) \equiv(\bar{c} \wedge d))$ can be written as $\bar{a}|b:: c| d$. This proportion is indeed obtained from the conditional proportion $a|b:: c| d$ by changing $a$ into $\bar{a}$. Thus, these 16 new equivalences between conditional objects are not of the form $a|b:: c| d$ (or equivalently $\bar{a}|b:: \bar{c}| d$ ) produced by the pattern of conditional proportions, but of a "mixed" form having an odd number of negated terms.

2.3.4. The 32 semi-hybrid proportions. One half of their expressions involve indicators of the same kind, while the other half requires equivalence between indicators of opposite kinds. They are of the form

$$
\begin{array}{ll}
\text { or } & S_{(a, b)} \equiv S_{(c, d)} \wedge S_{(a, b)}^{\prime} \equiv D_{(c, d)} \\
& S_{(a, b)} \equiv S_{(c, d)} \wedge D_{(a, b)} \equiv S_{(c, d)}^{\prime} \\
\text { or } & D_{(a, b)} \equiv D_{(c, d)} \wedge S_{(a, b)} \equiv D_{(c, d)}^{\prime} \\
\text { or } & D_{(a, b)} \equiv D_{(c, d)} \wedge D_{(a, b)}^{\prime} \equiv S_{(c, d)}
\end{array}
$$

They are listed in Table 11 in Annex A. There are 32 semi-hybrid proportions ( 8 of each kind: 4 choices for the first equivalence, times 2 choices for the element that is not of the same type as the three others $(D$ or $S)$ in the second equivalence). An example of semi-hybrid proportion is $((a \wedge b) \equiv(c \wedge d)) \wedge((\bar{a} \wedge \bar{b} \equiv(\bar{c} \wedge d))$.

Applying a change from $a$ to $\bar{a}$ (and $\bar{a}$ to $a$ ), or applying a similar transformation with respect to $b, c$, or $d$, turns a semi-hybrid proportion into a semi-hybrid proportion (since as already said, negating anyone of the two terms of a dissimilarity indicator turns it into a similarity indicator, and conversely). This contrasts with the hybrid proportion class which is not closed under such a transformation.

2.3.5. The 48 degenerated proportions. In all the above categories, the 4 terms related by equivalence symbols should be all distinct. In degenerated proportions, there are only 3 different terms and it is simpler to come back to our initial notation. With this notation, these proportions are of the form

$$
\begin{aligned}
\text { or } \quad I_{(a, b)} & \equiv I_{(c, d)} \wedge I_{(a, b)} \equiv I_{(c, d)}^{\prime} \\
I_{(a, b)} & \equiv I_{(c, d)} \wedge I_{(a, b)}^{\prime} \equiv I_{(c, d)}
\end{aligned}
$$

They are listed in Table 12 in Annex A. Their number is easy to compute: we have to choose $I_{(a, b)}$ among 4 indicators and then to choose 2 distinct indicators among 4 pertaining to $(c, d)$ : we then get $4 * 6=24$ proportions of the first form. The same reasoning with the second kind of expression leads to a total of 48 degenerated proportions. Note that the change from $a$ to $\bar{a}$ (and $\bar{a}$ to $a$ ), or a similar transformation with respect to $b, c$, or $d$, turns a degenerated proportion into a degenerated proportion. 
It can be seen that degenerated proportions always involve a mutual exclusiveness condition between 2 positive or negative literals pertaining to either the pair $(a, b)$ or the pair $(c, d)$. Indeed, if we consider the first form, we get $I_{(a, b)} \equiv I_{(c, d)}$ on the one hand, and $I_{(c, d)} \equiv I_{(c, d)}^{\prime}$ on the other hand, i.e. an equivalence between two syntactically distinct indicators pertaining to the same pair $(c, d)$. There are 6 cases only:

- $(\bar{c} \wedge d) \equiv(c \wedge \bar{d})$ iff $c \equiv d$

- $(c \wedge d) \equiv(\bar{c} \wedge \bar{d})$ iff $c \equiv \bar{d}$

- $(c \wedge d) \equiv(c \wedge \bar{d})$ iff $c \equiv \perp$

- $(c \wedge d) \equiv(\bar{c} \wedge d)$ iff $d \equiv \perp$

- $(\bar{c} \wedge d) \equiv(\bar{c} \wedge \bar{d})$ iff $\bar{c} \equiv \perp$

- $(c \wedge \bar{d}) \equiv(\bar{c} \wedge \bar{d})$ iff $\bar{d} \equiv \perp$

Thus, we also have $I_{(a, b)} \equiv \perp$ (since we have $I_{(c, d)} \equiv \perp$ and $I_{(c, d)}^{\prime} \equiv \perp$ ), which expresses a mutual exclusiveness condition. Since we have 4 possible choices for $I_{(a, b)}$, it yields $4 \times 6=24$ distinct proportions, and exchanging $(a, b)$ with $(c, d)$ gives the 24 other degenerated proportions.

Thus, generally speaking, degenerated proportions correspond to a mutual exclusiveness condition between component(s) or negation of component(s) of one of the pairs $(a, b)$ or $(c, d)$, together with

- either an identity condition pertaining to the other pair,

- or a tautology condition on one of the literals of the other pair without any constraint on the other literal.

\subsection{Two other classes of interest: uniform and weakly uniform proportions}

As recalled in the introduction, a numerical proportion states the equality of 2 ratios $\frac{a}{b}=\frac{c}{d}$, or of 2 differences $a-b=c-d$, comparing quantities of the same types, namely ratios or differences. In no case, we mix them as, e.g., $\frac{a}{b}=c \times d$ or $a-b=c+d$. In logical proportions the equality of quantities is converted into equivalence. Then, if we want to completely fit with the spirit of numerical proportions, we may insist on using the same indicators on each side of the equivalence symbol. It means that when we have chosen 2 distinct indicators for the pair $(a, b)$, we should stick to the same indicators for the other pair $(c, d)$. For instance, let us assume that we choose $(a \wedge b, \bar{a} \wedge b)$ as indicators for the pair $(a, b)$, then we have to use the indicators $(c \wedge d, \bar{c} \wedge d)$ for the pair $(c, d)$. Strictly speaking, we have exactly 4 uniform equivalences:

- $a \wedge b \equiv c \wedge d$

- $\bar{a} \wedge b \equiv \bar{c} \wedge d$

- $a \wedge \bar{b} \equiv c \wedge \bar{d}$

- $\bar{a} \wedge \bar{b} \equiv \bar{c} \wedge \bar{d}$

Since we need 2 equivalences to build up a proportion, we get exactly $\left[\begin{array}{l}4 \\ 2\end{array}\right]=6$ uniform proportions, thus built up with a pair of uniform equivalences. As can be seen, $A$ and $P$ are uniform, but $R$ and $I$ are not uniform. 
The 4 remaining uniform proportions are given below; note that they are conditional proportions:

- $(a \wedge b \equiv c \wedge d) \wedge(\bar{a} \wedge b \equiv \bar{c} \wedge d) ;(a \wedge b \equiv c \wedge d) \wedge(a \wedge \bar{b} \equiv c \wedge \bar{d})$

- $(\bar{a} \wedge \bar{b} \equiv \bar{c} \wedge \bar{d}) \wedge(\bar{a} \wedge b \equiv \bar{c} \wedge d) ;(\bar{a} \wedge \bar{b} \equiv \bar{c} \wedge \bar{d}) \wedge(a \wedge \bar{b} \equiv c \wedge \bar{d})$

Relaxing a little bit the constraint of uniformity by allowing us to switch the indicators in the equivalences, we are led to 12 weakly uniform proportions: we can exchange the right hand side of the equivalences, we get $6 \times 2=12$ such proportions. $R$ and $I$ are weakly uniform. It has to be noticed that, since we cannot build a weakly uniform proportion with only 3 distinct indicators, a degenerated proportion cannot be weakly uniform. If we go one step further, we may decide to consider proportions, not only satisfying the previous uniformity constraint, but also where the equivalences use only one type of indicators (similarity or dissimilarity). In that case, we have only 2 candidates set of indicators for the pair $(a, b)$, each candidate set for $(a, b)$ leading to a unique candidate set for $(c, d)$, then to the 2 uniform proportions $A$ and $P$. Allowing weak uniformity adds $R$ and $I$ to this list and we are back to the "homogeneous" proportions.

\section{Truth tables of logical proportions}

Since $a, b, c, d$ are Boolean variables, logical proportions can be considered as quaternary Boolean formulas, whose semantics is given via their truth tables (which then have $2^{4}=16$ lines). We start by considering the case of the 4 homogeneous proportions $A, R, P, I$ defined in section 2.3.1, since it is the smallest family, and it contains the "Analogy" proportion. Then, we shall establish two general results pertaining to the truth tables of the whole class of logical proportions.

\subsection{Homogeneous proportions truth tables}

Starting from their Boolean expressions, it is an easy game to build up the truth tables of proportions $A, R, P, I$ : they are exhibited in Table 1 , where only the valuations leading to the truth value 1 , are shown. This means that all the other ones lead to the truth value 0 . There is one fact immediately appearing: only 6 valuations among 16 in the tables lead to a truth value 1 . We also observe that there are only 8 distinct 4 -tuples that appear in Table 1 . This emphasizes their collective coherence as the whole class of homogeneous proportions. Moreover, they go by pairs where 0 and 1 are exchanged, thus pointing out their "code independency".

It is interesting to take a closer look at the truth tables of the four homogeneous proportions. First, one can observe in Table 1, that 8 possible valuations for $(a, b, c, d)$ never appear among the patterns that make $A, R, P$, or $I$ true: these 8 valuations are of the form $x x x y, x x$ y $x, x$ y $x x$, or $y x x x$ with $x \neq y$ and $(x, y) \in\{0,1\}^{2}$. As can be seen, it corresponds to situations where $a=b$ and $c \neq d$, or $a \neq b$ and $c=d$, i.e., similarity holds between the components of one of the pairs, and dissimilarity holds in the other pair. Moreover, the truth table of each of the four homogeneous proportions, is built in the same manner: 


\begin{tabular}{|c|c|c|c|c|c|c|c|c|c|c|c|c|c|c|}
\hline \multicolumn{3}{|c|}{ A } & \multicolumn{4}{|c|}{$\mathbf{R}$} & \multicolumn{4}{|c|}{$\mathbf{P}$} & \multicolumn{4}{|c|}{ I } \\
\hline $0 \quad 0$ & 0 & 0 & 0 & 0 & 0 & 0 & 0 & 0 & 0 & 0 & 1 & 1 & 0 & 0 \\
\hline 1 & 1 & 1 & 1 & 1 & 1 & 1 & 1 & 1 & 1 & 1 & 0 & 0 & 1 & 1 \\
\hline 0 & 1 & 1 & 0 & 0 & 1 & 1 & 1 & 0 & 0 & 1 & 1 & 0 & 0 & 1 \\
\hline 1 & 0 & 0 & 1 & 1 & 0 & 0 & 0 & 1 & 1 & 0 & 0 & 1 & 1 & 0 \\
\hline 1 & 0 & 1 & 0 & 1 & 1 & 0 & 0 & 1 & 0 & 1 & 0 & 1 & 0 & \\
\hline 10 & 1 & 0 & 1 & 0 & 0 & 1 & 1 & 0 & 1 & 0 & 1 & 0 & 1 & \\
\hline
\end{tabular}

TABLE 1. Truth tables: Analogy, Reverse analogy, Paralogy, Inverse paralogy

1. 2 lines of the table correspond to the characteristic pattern of the proportion; namely the two lines where one of the two equivalences in its definition holds true under the form $1 \equiv 1$ (rather than $0 \equiv 0$ ). Thus,

- $A$ is characterized by the pattern $x$ y $x$ y (corresponding to valuations 1010 and 010 1), i.e. we have the same difference between $a$ and $b$ as between $c$ and $d$;

- $R$ is characterized by the pattern yxxy (corresponding to valuations 1001 and 0110 ), i.e. the differences between $a$ and $b$ and between $c$ and $d$ are in opposite directions;

- $P$ is characterized by the pattern $x \times x \times$ (corresponding to valuations 1111 and 000 0), i.e. what $a$ and $b$ have in common, $c$ and $d$ have it also;

- $I$ is characterized by the pattern $x x$ y $y$ (corresponding to valuations 1100 and 001 1), i.e. what $a$ and $b$ have in common, $c$ and $d$ do not have it, and conversely. Thus, the six lines of the truth table of $A$ that makes it true are induced by the characteristic patterns of $A, P$, and $I^{5}$, the six valuations that makes $P$ true are induced by the characteristic patterns of $P, A$, and $R$, and so on for $R$ and $I$.

2. the 4 other lines of the truth table of an homogeneous proportion $T$ are generated by the characteristic patterns of the two other proportions that are not opposed to $T$ (in the sense that $A$ and $R$ are opposed, as well as $P$ and $I$ ). For these four lines, the proportion holds true since its expression reduces to $(0 \equiv 0) \wedge(0 \equiv 0)$.

As an illustration of analogy and other homogeneous proportions, let us consider Figure 1 where a pair of items $(A, B)$ is shown together with an incomplete pair $(C$, ?). Each of the three pictures can be represented by a vector of Boolean variables acknowledging, in this order, the presence or not of outside squares, of outside circles, of an inside circle (upper position), of an inside hexagon (lower position), of hatching (upper position). Namely, $A$ (the first picture) corresponds to $(1,0$, $1,0,0), B$ (the second picture) to $(1,0,0,1,1), C$ (the third picture) to $(0,1$,

\footnotetext{
${ }^{5}$ The measure of analogical dissimilarity introduced in [50] is 0 for the valuations corresponding to the characteristic patterns of $A, P$, and $I$, maximal for the valuations corresponding to the characteristic patterns of $R$, and takes the same intermediary value for the 8 valuations characterized by one of the patterns $x x x y, x x y x, x y x x$, or $y x x x$.
} 
$1,0,0)$. Then, one may wonder if we can associate a 4 th item $D$ (represented as ?) to the 3 previous ones, using analogy or another homogeneous proportion. The situation can be thus summarized by the following table:

\begin{tabular}{cccccc} 
& out.squ. & out.cir. & ins.cir. & ins.hex. & hatc. \\
\hline$A$ & 1 & 0 & 1 & 0 & 0 \\
$B$ & 1 & 0 & 0 & 1 & 1 \\
$C$ & 0 & 1 & 1 & 0 & 0 \\
$D$ & $?$ & $?$ & $?$ & $?$ & $?$
\end{tabular}

TABLE 2. Boolean encoding of the illustrative example

Working feature by feature, this amounts to try to complete the 5 vectors $(1$, $1,0, ?),(0,0,1, ?),(1,0,1, ?),(0,1,0, ?)$, and $(0,1,0, ?)$ using the same homogeneous proportion, if possible. It can be checked on Table 1 that there are here two solutions, either using analogy, or inverse paralogy, yielding the unique description of $D$ as $(0,1,0,1,1)$, i.e., an item with outside circles, with an inside hexagon (lower position), and hatching (upper position). Note that if in the description, we add features such that "the figure has two parts", or "there is a triangle", we are also led to complete the following vectors $(1,1,1, ?)$ or $(0,0,0$, ?) respectively, and then only analogy would work.
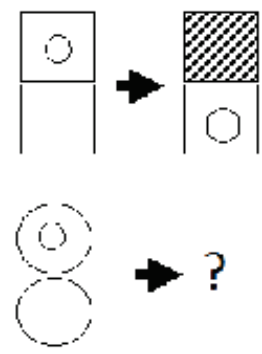

FiguRE 1. An illustration: Logical proportions at work

\subsection{Characterization of the truth tables of logical proportions}

The fact that the truth tables of the homogeneous proportions have only 6 lines making the proportion valid is not proper to this family, but is shared by the whole set of the 120 logical proportions. Indeed the following general result can be proved:

Proposition 1. The truth table of a logical proportion has 6 and only 6 valuations with truth value 1 . 
Proof (indication) $)^{6}$ : In fact, a simple equivalence $e q_{1}$ between indicators has exactly 10 lines leading to true. Since a logical proportion $T$ is the conjunction $e q_{1} \wedge e q_{2}$ of 2 equivalences between indicators, it appears from the previous property that $T$ has a maximum of 10 valid valuations and a minimum of 4 valid valuations. Obviously, adding $e q_{2}$ to $e q_{1}$ may only reduce the number of valid valuations for $T$. Starting from $e q_{1}$ and reasoning on the number of literals where $e q_{1}$ and $e q_{2}$ differ, we show that we keep only 6 valid valuations for $T$; see complete proof in Annex B.

It follows that the negation of a logical proportion is not a logical proportion (since such a negation has 10 valuations leading to true in its table).

As previously explained, due to the symmetry of the $\wedge$ operator, we have 120 proportions which are potentially semantically distinct. A question remains: can we reduce this number of semantically distinct proportions? The answer is negative, as now stated.

Proposition 2. The truth tables of the 120 proportions are all distinct.

Proof (indication): We shall show that, when 2 proportions $T \triangleq e q_{1} \wedge e q_{2}$ and $T^{\prime} \triangleq e q_{1}^{\prime} \wedge e q_{2}^{\prime}$ have the same truth table, they are syntactically identical (up to a permutation of the 2 equalities) i.e. $T={ }_{I d} T^{\prime}$. This is done by showing that in that case, every literal occurring in one of them appears in the other one; see complete proof in Annex B.

It appears that despite their similar structure, logical proportions are semantically distinct and then they cover distinct situations. This result may look all the more amazing as logical proportions are quite rare. Indeed we know that we have $\left(\begin{array}{l}16 \\ 6\end{array}\right)=16 \times 15 \times 14 \times 13 \times 12 \times 11 / 6 !=5765760 / 720=8008$ truth tables with exactly 6 valuations leading to 1 , while only 120 tables among them are the truth table of a logical proportion.

\section{Noticeable properties of subfamilies of logical proportions}

Thinking of proportions, and more particularly of symbolic proportions, naturally suggest properties of interest that might be expected, such as symmetry, or equation solving as already suggested in the introduction, or code independency also discussed previously. As we shall see in subsection 4.9, these properties are in fact the counterparts of properties satisfied by numerical proportions.

\subsection{Full identity}

Let us carry on the investigation of the truth tables in Table 1. A, $R$ and $P$ include the valuations 1111 and 0000: a common property is then satisfied by these 3

\footnotetext{
${ }^{6}$ We only give in the main text the proofs that are short, or that may be useful for the general understanding of the discussed matter. Otherwise they can be found in Annex B.
} 
proportions which can be stated as a simple axiom $T(a, a, a, a)$ (where $T$ denotes a proportion). This property means that when $a, b, c, d$ have a common truth value, then the proportion is satisfied whatever this truth value. We call this property full identity. Obviously $I$ does not satisfy this property because of the exchange of the negation operator between pairs $(a, b)$ and $(c, d)$ in the definition. Still this property can be considered as intuitively appealing, and it is interesting to identify the proportions that satisfy it. The following result can be established:

Proposition 3. There are only 15 proportions satisfying full identity: 3 of them are homogeneous (they are $A, R$, and $P$ ), 8 of them are conditional proportions and the 4 remaining ones are degenerated. (these proportions are shown in Annex $A$ Table 13).

Proof : See Annex B.

Then a new question arises: Are there proportions satisfying only "half" of the full identity property, i.e., with truth value 1 for valuation 1111 and 0 for 0000 , or vice-versa? We have the following result:

Proposition 4. There are 30 proportions validated with 1111 but not with 0000. Dually, there are also 30 proportions validated with 0000 but not with 1111.

Proof: See Annex B.

Each of the above two categories satisfying "half of full identity" contains 4 hybrid proportions, 12 semi-hybrid ones, and 14 degenerated ones. The four hybrid ones satisfying 1111 are defined by

- $(\bar{a} \wedge \bar{b} \equiv c \wedge \bar{d}) \wedge(\bar{a} \wedge b \equiv \bar{c} \wedge \bar{d})$, corresponding to conditional $b|\bar{a}:: c| \bar{d}$,

- $(\bar{a} \wedge \bar{b} \equiv c \wedge \bar{d}) \wedge(a \wedge \bar{b} \equiv \bar{c} \wedge \bar{d})$, corresponding to conditional $a|\bar{b}:: c| \bar{d}$,

- $(\bar{a} \wedge \bar{b} \equiv \bar{c} \wedge d) \wedge(\bar{a} \wedge b \equiv \bar{c} \wedge \bar{d})$, corresponding to conditional $b|\bar{a}:: d| \bar{c}$,

- $(\bar{a} \wedge \bar{b} \equiv \bar{c} \wedge d) \wedge(a \wedge \bar{b} \equiv \bar{c} \wedge \bar{d})$, corresponding to conditional $a|\bar{b}:: d| \bar{c}$.

The four hybrid ones satisfying 0000 correspond to $\bar{a}|b:: c| d, \bar{b}|a:: c| d, \bar{a}|b:: d| c$, $\bar{b}|a:: d| c$. As a consequence of the previous results, there are $45(=120-15-30-$ 30) proportions that are false for both valuations 1111 and 0000. They include 1 homogeneous proportion (the inverse paralogy), 8 conditional ones, 12 hybrid ones, 8 semi-hybrid ones and 16 degenerated ones. Among hybrid ones, let us mention the presence of the proportions corresponding to analogy (or paralogy) where one literal is negated, such as $A(\bar{a}, b, c, d)$ for instance.

\subsection{Reflexivity, reverse reflexivity and sameness}

It can be easily checked that $A(a, b, a, b)$ holds (which means that the analogical proportion satisfies "a is to b as a is to b"). We may refer to this property as reflexivity of the proportion. Obviously, reflexivity entails full identity. There are 
two other natural ways ${ }^{7}$ to strengthen full identity, namely, $T(a, b, b, a)$ referred as reverse reflexivity, and $T(a, a, b, b)$ referred as sameness (meaning "a is to a as b is to b" for an analogical proportion, for which $A(a, a, b, b)$ holds).

We are thus led to look for the logical proportions $T$ satisfying at least one of the 3 properties: $T(a, a, b, b), T(a, b, a, b)$, or $T(a, b, b, a)$. Since, such a proportion should obviously satisfy full identity, we have a maximum of 15 such proportions for each of the 3 properties. In fact, it can be shown that:

\section{Proposition 5.}

- There is no proportion simultaneously satisfying the 3 properties.

- $A$ is the only proportion to satisfy $T(a, a, b, b)$ and $T(a, b, a, b)$.

- $R$ is the only proportion to satisfy $T(a, a, b, b)$ and $T(a, b, b, a)$.

- $P$ is the only proportion to satisfy $T(a, b, a, b)$ and $T(a, b, b, a)$.

- 6 proportions satisfy $T(a, b, a, b)$, including $A$ and $P$ but not $R$ and $I$.

- 6 proportions satisfy $T(a, b, b, a)$, including $P$ and $R$ but not $A$ and $I$.

- 6 proportions satisfy $T(a, a, b, b)$, including $A$ and $R$ but not $P$ and $I$.

Proof: This is an easy proof for the first 4 statements since each property generates a set of 4 valid valuations (and two of them yield 6 valid valuations). For instance, having the simultaneous satisfaction of the 3 properties leads to a truth table where the 8 valuations 0000, 1111, 1010, 0101, 0110, 1001, 0011, 1100 are valid: then this cannot be the truth table of a logical proportion. Let us consider the fifth statement. Given a logical proportion $T(a, b, c, d)$ of the form $\left(I_{(a, b)}^{1} \equiv I_{(c, d)}^{2}\right) \wedge$ $\left(I_{(a, b)}^{3} \equiv I_{(c, d)}^{4}\right)$, reflexivity enforces the two equivalences $I_{(a, b)}^{1} \equiv I_{(a, b)}^{2}$ and $I_{(a, b)}^{3} \equiv$ $I_{(a, b)}^{4}$. This tells us that the indicators $I^{1}$ and $I^{2}$ coincide, as well as $I^{3}$ and $I^{4}$. Since we have four indicators, there are four such equivalences $a \wedge b \equiv c \wedge d$, $a \wedge \bar{b} \equiv c \wedge \bar{d}, \bar{a} \wedge b \equiv \bar{c} \wedge d, \bar{a} \wedge \bar{b} \equiv \bar{c} \wedge \bar{d}$. Since a logical proportion is defined from two distinct, non-ordered equivalences, there are $4 \times 3 / 2=6$ proportions satisfying $T(a, b, a, b)$. They are given in Table 3 where we omit the $\wedge$ symbol for the benefit of more compact notations. We recognize the proportions $A, P$, and the 4 conditional proportions $b|a:: d| c, a|b:: c| d, a|\bar{b}:: c| \bar{d}$, and $b|\bar{a}:: d| \bar{c}$. Similar proofs can be made for the two last statements; see Annex B.

$$
\begin{gathered}
a \bar{b} \equiv c \bar{d} \quad \bar{a} b \equiv \bar{c} d \quad(A) \| a b \equiv c d \quad \bar{a} \bar{b} \equiv \bar{c} \bar{d} \\
a b \equiv c d \quad(P) \\
a \bar{b} \equiv c \bar{d}\|a b \equiv c d \quad \bar{a} b \equiv \bar{c} d\| a \bar{b} \equiv c \bar{d} \quad \bar{a} \bar{b} \equiv \bar{c} \bar{d} \| \bar{a} b \equiv \bar{c} d \quad \bar{a} \bar{b} \equiv \bar{c} \bar{d}
\end{gathered}
$$

TABLE 3. The 6 proportions satisfying reflexivity

\footnotetext{
${ }^{7}$ Strictly speaking, one might also wonder about other strengthening of full identity such as $T(a, a, a, b)$. It turns out that with an odd number of $a$ (and $b$ ), there is no logical proportions satisfying such property.
} 


\subsection{Symmetry}

Observing Table 1, there is a common property clearly satisfied by $P, A, R$ and $I$, the so-called symmetry property which can be stated as (where $T$ denotes a proportion):

$$
T(a, b, c, d) \rightarrow T(c, d, a, b)
$$

This property tells us that we can exchange the pair $(a, b)$ with the pair $(c, d)$ in the logical proportion $T$. This is an expected property for analogical proportion for instance, since if $a$ is to $b$ as $c$ is to $d$ holds, we want $c$ is to $d$ as a is to $b$ to hold as well. Nevertheless, symmetry is a quite rare property as stated in the following result:

Proposition 6. There are only 12 proportions satisfying symmetry. Apart from $A, R, P, I$ (homogeneous proportions), there are 4 conditional proportions and 4 hybrid proportions satisfying symmetry. (These proportions are shown in Table 14 in Annex A)

Proof: See Annex B.

Then it can be checked in Table 14 that the 6 uniform logical proportions are symmetrical proportions. But the 6 other symmetrical proportions are not weakly uniform.

This result points out that we have to be careful: despite the definitions of proportions are based on equalities between 2 terms (involving negation and conjunction), the symmetrical nature of an equality is not sufficient to ensure the symmetry of all logical proportions. Only few of them satisfy the symmetry property (12 over 120 ), although it may be considered as being a desirable property for something which is called 'proportion'. Note that none of the semi-hybrid proportions and none of the degenerated ones is symmetrical.

\subsection{Central permutation and other permutations}

As highlighted in the introduction, a well known property of mathematical proportions, often called central permutation property, is the fact that we can exchange the 2 central elements of such a proportion without changing its truth value. We can check on the truth table that this property is still satisfied when dealing with its logical counterpart, namely analogical proportion. The central permutation property is formally expressed by:

$$
T(a, b, c, d) \rightarrow T(a, c, b, d)
$$

We have the following result:

Proposition 7. There are exactly 16 proportions satisfying the central permutation property. Only 2 homogeneous proportions $A$ and I satisfy it.

Proof: See Annex B. 
We may also consider other permutations: since we have 4 variables, there are exactly 6 ways to exchange 2 variables among 4 . Notably, it can be checked that:

- $A$ and $I$ are the only homogeneous proportions to satisfy central and external permutations, namely, $T(a, b, c, d) \rightarrow T(a, c, b, d)$ and $T(a, b, c, d) \rightarrow$ $T(d, b, c, a)$;

- $P$ and $I$ are the only homogeneous proportions to satisfy the permutations $T(a, b, c, d) \rightarrow T(b, a, c, d)$ and $T(a, b, c, d) \rightarrow T(a, b, d, c) ;$

- $R$ and $I$ are the only homogeneous proportions to satisfy the permutations $T(a, b, c, d) \rightarrow T(c, b, a, d)$ and $T(a, b, c, d) \rightarrow T(a, d, c, b)$.

Inverse paralogy is thus the unique homogeneous proportion to satisfy the 6 permutations. In fact, a stronger result holds concerning this logical proportion.

Proposition 8. Inverse paralogy is the unique logical proportion to satisfy the 6 permutations.

Proof: It is easy to check that these permutations induce a partition of the set of valuations into 5 classes, each of them being closed for these 6 permutations:

- the class $\{0000\}$ and the class $\{1111\}$

- the class $\{0111,1011,1101,1110\}$

- the class $\{1000,0100,0010,0001\}$

- the class $\{0101,1100,0011,1010,1001,0110\}$

Taking into account that a logical proportion is true for only 6 valuations (Proposition 1), we only have 3 options: a proportion valid for $\{0000\},\{1111\}$ and $\{0111,1011,1101,1110\}$, or for $\{0000\},\{1111\}$ and $\{1000,0100,0010,0001\}$, or for $\{0101,1100,0011,1010,1001,0110\}$. It appears that the latter class is just the truth table of inverse paralogy. Lemma 5 (see annex B) allows us to achieve the proof since there is no logical proportion valid for the class $\{0111,1011,1101,1110\}$ or for the class $\{1000,0100,0010,0001\}$.

The two following propositions lay bare the links between the different permutations and the homogeneous proportions.

\section{Proposition 9.}

- $A$ and $I$ are the only logical proportions satisfying symmetry and being stable for permutation $p 23$, i.e. the permutation of the means. The same result holds replacing $p 23$ by $p 14$ (permutation of extremes).

- $P$ and $I$ are the only logical proportions satisfying symmetry and being stable for permutation $p 12$. The same result holds replacing $p 12$ by $p 34$.

- There are 10 proportions including only 2 homogeneous ${ }^{8}$, namely $R$ and $I$, satisfying symmetry and being stable for permutation $p 24$. The same result holds replacing $p 13$ by $p 24$.

Proof: See Annex B.

\footnotetext{
${ }^{8}$ We made a mistake in a previous paper [65], where we suggested that the 2 homogeneous $R$ and $I$ were the only ones to satisfy this property.
} 
We have the following result:

Proposition 10. $A$ is the unique proportion satisfying $T(a, b, a, b)$ and $p 23$ (and thus also $T(a, a, b, b)) . P$ is the unique proportion satisfying $T(a, b, a, b)$ and $p 34$ (and thus also $T(a, b, b, a)) . R$ is the unique proportion satisfying $T(a, a, b, b)$ and $p 24$ (and thus also $T(a, b, b, a)$ ).

Proof: Let us consider the first statement for instance. $T(a, b, a, b)$ implies that $T(0,0,0,0), T(1,1,1,1), T(1,0,1,0)$ and $T(0,1,0,1)$ hold. Adding the fact that $T$ is stable for the permutation of the mean $p 23$, we get that $T(1,1,0,0)$ and $(T(0,0,1,1)$ hold as well, leading to the truth table of $A$.

A similar reasoning is still valid for $P$ and $R$ and achieves the proof.

\subsection{Code independency}

We now consider an important property which has been already suggested and that we call code independency: this property can be observed in Table 1. Indeed from a semantical viewpoint, it may be meaningful for a proportion to be independent from the coding convention (i.e., true represented by 1 and false by 0 ). That is why if we switch the values 0 and 1 in the coding of a given valuation, the truth value of the proportion should remain the same. This is formally expressed by the code independency property:

$$
T(a, b, c, d) \rightarrow T(\bar{a}, \bar{b}, \bar{c}, \bar{d})
$$

Unfortunately, many logical proportions do not satisfy code independency, and we have the following result:

Proposition 11. There are exactly 8 proportions satisfying the code independency property: the 4 homogeneous proportions $A, R, P, I$, and 4 hybrid proportions.

Proof: See Annex B.

We exhibit these 4 hybrid proportions in Table 4 (where we use the same convention as in Table 3). It is remarkable that, apart from $A, R, P, I$ which are the

$$
a b \equiv c \bar{d} \quad \bar{a} \bar{b} \equiv \bar{c} d\|a b \equiv \bar{c} d \quad \bar{a} \bar{b} \equiv c \bar{d}\| \bar{a} b \equiv c d \quad a \bar{b} \equiv \bar{c} \bar{d} \| a \bar{b} \equiv c d \quad \bar{a} b \equiv \bar{c} \bar{d}
$$

TABLE 4 . The 4 hybrid proportions satisfying code independency

homogeneous proportions, we get the hybrid proportions of Table 4 simply by negating one of the 4 variables in the equivalences. For instance, the first hybrid one corresponds to the definition of $A(a, \bar{b}, c, d)$, the second to $R(a, \bar{b}, c, d)$, the third one to $I(a, \bar{b}, c, d)$, and the fourth one to $P(a, \bar{b}, c, d)$.

Moreover, the inspection of Table 4 reveals that none of these 4 hybrid proportions are symmetrical. Then, this remark together with Proposition 11 lead to the following remarkable proposition. 
Proposition 12. There are exactly 4 proportions satisfying code independency and symmetry. They are the 4 homogeneous proportions $A, R, P, I$

This result confirms the central role played by the proportions $A, R, P$ and $I$ as the most important ones.

\subsection{Transitivity}

If we remember that analogical proportion describes an equality between ratios, it is natural to expect a kind of transitivity property to hold for analogy $A$ and more generally for some other proportion $T$ which could be stated as follows:

$$
T(a, b, c, d) \wedge T(c, d, e, f) \rightarrow T(a, b, e, f)
$$

It can be checked that the analogical proportion $A$, as well as the paralogical proportion $P$, are transitive in that sense. The question is to find how many logical proportions satisfy this property. We have the following result:

Proposition 13. There are 54 transitive proportions: 2 homogeneous $A$ and $P, 4$ conditional proportions, namely $(a b \equiv c d) \wedge(a \bar{b} \equiv c \bar{d}) ;(a b \equiv c d) \wedge(\bar{a} b \equiv \bar{c} d) ;(a \bar{b} \equiv$ $c \bar{d}) \wedge(\bar{a} \bar{b} \equiv \bar{c} \bar{d}) ;(\bar{a} b \equiv \bar{c} d) \wedge(\bar{a} \bar{b} \equiv \bar{c} \bar{d})$, and the 48 degenerated proportions.

Proof: See Annex B.

The fact that the 48 degenerated proportions are transitive should not come as a surprise, as it is a straightforward consequence of their syntactic pattern structure involving only three indicators. The transitivity of the 4 conditional proportions is due to the fact that they express equivalences between conditional objects, namely $b|a:: d| c, a|b:: c| d, a|\bar{b}:: c| \bar{d}$, and $b|\bar{a}:: d| \bar{c}$.

Moreover, it is worth noticing that for logical proportions, reflexivity entails both symmetry (as it can be checked from Table 3 and Table 14), and transitivity (due to the above proposition). In other words, a reflexive logical proportion is an equivalence relation.

Besides, having a closer look on the homogeneous proportions, we can easily build Table 5 which gives what $T(a, b, c, d) \wedge T(c, d, e, f)$ entails for the 4 homogeneous proportions.

\begin{tabular}{|c|c|}
\hline chaining & result \\
\hline$A \wedge A$ & $A$ \\
\hline$R \wedge R$ & $A$ \\
\hline$P \wedge P$ & $P$ \\
\hline$I \wedge I$ & $P$ \\
\hline$A \wedge R$ & $R$ \\
\hline$P \wedge I$ & $I$ \\
\hline
\end{tabular}

TABLE 5. Chaining properties for homogeneous proportions 


\subsection{Other interesting properties involving negation}

It is quite tempting to consider $a$ is to $b$ as $\bar{a}$ is to $\bar{b}$ should hold or even $a$ is to $\bar{a}$ as $b$ is to $\bar{b}$ just because of a formal symmetry. But, for instance, 0110 does not validate the analogical proportion, which means that none of these two properties is satisfied by the analogical proportion. Nevertheless, for a given proportion $T$, it is worth to consider if $T(a, b, \bar{a}, \bar{b}), T(a, \bar{a}, b, \bar{b})$ or even $T(a, b, \bar{b}, \bar{a})$ holds.

- $T(a, b, \bar{a}, \bar{b})$ will be called semi-mirroring property and

- $T(a, \bar{a}, b, \bar{b})$ will be called negation-compatibility property.

- $T(a, b, \bar{b}, \bar{a})$ will be called exchange-mirroring property.

Proposition 14. A logical proportion satisfying 2 properties among semi-mirroring, negation-compatibility and exchange-mirroring satisfies the remaining one, and is unique. This is the inverse paralogy $I$.

Proof: Let us choose for instance semi-mirroring and negation-compatibility. First of all, we can observe that, for a proportion $T$ to satisfy semi-mirroring, means the 4 valuations 1010,1001,0110,0101 are valid. For negation-compatibility to be satisfied, the 4 valuations $1100,0011,1001,0110$ should be valid. Then the truth table of a proportion satisfying both properties should contains all these valuations i.e. 1010,1001,0110,0101,1100,0011: this is the truth table of inverse paralogy $I$. A similar reasoning applies for the remaining of the proposition.

When we consider the 3 properties separately, we get the following result:

Proposition 15. Among the 120 logical proportions, only 6 (among which the homogeneous $R$ and $I$ ) satisfy semi-mirroring, only 6 (among which the homogeneous $P$ and $I$ ) satisfy negation-compatibility, and only 6 (among which the homogeneous $A$ and I) satisfy exchange-mirroring.

Proof: See Annex B.

\subsection{Equation solving}

As said in the introduction, the idea of proportion is closely related to the idea of extrapolation, i.e. to guess/compute a new value on the ground of existing values. In other words, if for some reason, it is believed or known that a proportion holds between 4 binary items, 3 of them being known, then one may try to infer the value of the 4 th one, at least in the case this extrapolation leads to a unique value.

For a proportion $T$, there are exactly 6 valuations $v$ such that $v(T(a, b, c, d))=$ 1. In our context, the problem can be stated as follows. Given a logical proportion $T$ and a valuation $v$ such that $v(a), v(b), v(c)$ are known, does it exist a Boolean value $x$ such that $v(T(a, b, c, d))=1$ when $v(d)=x$, and in that case, is this value unique?

We will refer to this problem as "the equation solving problem", and for the sake of simplicity, a propositional variable $a$ is denoted as its truth value $v(a)$, and we use the equational notation $T(a, b, c, x)=1$, where $x \in\{0,1\}$ is unknown. First of all, it is easy to see that there are always cases where the equation has no 
solution. Indeed, the triple $a, b, c$ may take $2^{3}=8$ values, while any proportion $T$ is true only for 6 distinct valuations, leaving at least 2 cases with no solution. For instance, when we deal with analogy $A$, the equations $A(1,0,0, x)$ and $A(0,1,1, x)$ have no solution.

We first focus on the 4 homogeneous logical proportions $A, R, P, I$ that have been previously highlighted. We have the following results

\section{Proposition 16.}

The analogical equation $A(a, b, c, x)$ is solvable iff $(a \equiv b) \vee(a \equiv c)$ holds.

The reverse analogical equation $R(a, b, c, x)$ is solvable iff $(b \equiv a) \vee(b \equiv c)$ holds.

The paralogical equation $P(a, b, c, x)$ is solvable iff $(c \equiv b) \vee(c \equiv a)$ holds.

In each of the three above cases, when it exists, the unique solution is given by $x=c \equiv(a \equiv b)$, i.e. $x=a \equiv b \equiv c$.

The inverse paralogical equation $I(a, b, c, x)$ is solvable iff $(a \not \equiv b) \vee(b \not \equiv c)$ holds. In that case, the unique solution is $x=c \not \equiv(a \not \equiv b)$.

Proof: By immediate investigation of the truth tables.

As we can see, the first 3 homogeneous proportions $A, R, P$ behave similarly. Still, their conditions of equation solvability differ. Moreover, it can be checked that at least 2 of these proportions are always simultaneously solvable. Besides, when they are solvable, there is a common expression that yields the solution. This suggests a close relationship between $A, R$, and $P$. This strong link between these 3 homogeneous proportions will be made clear thanks to Proposition 21 in the next section. This contrasts with proportion $I$ which in some sense behaves in an opposite manner. This will be also made clearer in the next section.

Generally speaking, it should be clear that the unicity of the solution of an equation $T(a, b, c, x)=1$ when it exists, is unique if and only if $T$ is such that each of the 6 lines of its truth table starts with a different triple of values for $a, b, c$. We have the following result.

Proposition 17. There are 64 proportions for which the solution is always unique when it exists, and 56 proportions for which the equation $T(a, b, c, x)=1$ may have 2 solutions for some entries. These 56 proportions divide into 8 conditional ones, 8 hybrid ones, 8 semi-hybrid ones, and 32 degenerated ones.

Proof: see Annex B.

As already said, homogeneous proportions $A, R, P$ and $I$ always lead to a unique solution when it exists. Remarkably enough, this is also true for half of the conditional ones (e.g., $b|a:: \bar{c}| \bar{d}$, which is true for 1100, 1010, 0111, 0101, 0011, 0001), and false for the other half (e.g. $b|a:: d| c$, which is true for 1111, 1010, 0101, 0100, 0001, 0000).

As we have seen, the 4 homogeneous proportions $A, R, P, I$ not only enjoy interesting properties but they are, in some sense, the easiest to interpret. This 
is why we further investigate these proportions and their links in the following section.

Table 6 summarizes the results obtained regarding the potential properties of the logical proportions. In the table, we provide the number of proportions satisfying the target property and due to their particular status, we specify which are the homogeneous ones if any.

\begin{tabular}{|c|c|c|c|}
\hline Property name & Formal definition & nb prop. & Homoge. \\
\hline full identity & $T(a, a, a, a)$ & 15 & $A, R, P$ \\
\hline 1-full identity & $T(1,1,1,1) \wedge \neg T(0,0,0,0)$ & 30 & none \\
\hline 0-full identity & $T(0,0,0,0) \wedge \neg T(1,1,1,1)$ & 30 & none \\
\hline reflexivity & $T(a, b, a, b)$ & 6 & $A, P$ \\
\hline reverse reflexivity & $T(a, b, b, a)$ & 6 & $R, P$ \\
\hline sameness & $T(a, a, b, b)$ & 6 & $A, R$ \\
\hline symmetry & $T(a, b, c, d) \rightarrow T(c, d, a, b)$ & 12 & $A, R, P, I$ \\
\hline means permut. & $T(a, b, c, d) \rightarrow T(a, c, b, d)$ & 16 & $A, I$ \\
\hline extremes permut. & $T(a, b, c, d) \rightarrow T(d, b, c, a)$ & 16 & $A, I$ \\
\hline all permut. & $\forall i, j, T(a, b, c, d) \rightarrow T\left(p_{i, j}(a, b, c, d)\right)$ & 1 & $I$ \\
\hline transitivity & $T(a, b, c, d) \wedge T(c, d, e, f) \rightarrow T(a, b, e, f)$ & 54 & $A, P$ \\
\hline code independ. & $T(a, b, c, d) \rightarrow T(\bar{a}, \bar{b}, \bar{c}, \bar{d})$ & 8 & $A, R, P, I$ \\
\hline semi-mirror. & $T(a, b, \bar{a}, \bar{b})$ & 6 & $R, I$ \\
\hline exchange mirror. & $T(a, b, \bar{b}, \bar{a})$ & 6 & $A, I$ \\
\hline negation compatib. & $T(a, \bar{a}, b, \bar{b})$ & 6 & $P, I$ \\
\hline
\end{tabular}

TABlE 6. Properties of logical proportions

As can be seen from Table 6, homogeneous proportions look especially interesting, since they enjoy many properties. It is why the next main section is devoted to a deeper investigation of these proportions. Before that, we close the current section with a discussion that parallels logical proportions and numerical proportions in terms of properties. Again, as we are going to see, homogeneous proportions stand out.

\subsection{To what extent logical proportions are proportions}

Since numerical proportions are paradigmatic of the idea of a proportion, it is worth understanding why the logical proportions discussed in this paper really deserve the status of "proportion". In the numerical case, due to the symmetry of the $=$ operator and to the properties of the multiplication (for geometric proportions) or addition (for arithmetic proportions), these proportions both enjoy a set of well known properties that we recall here in the case of geometric proportions (a similar parallel could be made with arithmetic proportions, using opposites as inverses). 
1. When $\frac{a}{b}=\frac{c}{d}$ holds, then $\frac{c}{d}=\frac{a}{b}$ holds as well. Its counterpart for logical proportions is formally expressed via the "symmetry property", i.e.

$$
T(a, b, c, d) \rightarrow T(c, d, a, b)
$$

2. There is a well-known property, pertaining to "the permutation of extremes and means", which in fact covers a pair of properties:

- means's permutation: if $\frac{a}{b}=\frac{c}{d}$ holds then $\frac{a}{c}=\frac{b}{d}$ holds;

- extremes's permutation: if $\frac{a}{b}=\frac{c}{d}$ holds then $\frac{d}{b}=\frac{c}{a}$ holds.

The counterpart of these two properties in the logical setting is expressed as

$$
T(a, b, c, d) \rightarrow T(a, c, b, d) \text { for the means's permutation }
$$

and

$$
T(a, b, c, d) \rightarrow T(d, b, c, a) \text { for the extremes's permutation }
$$

3. With geometric proportions, we also have $\frac{a}{b}=\frac{c}{d}$ implies $\frac{\frac{1}{a}}{\frac{1}{b}}=\frac{\frac{1}{c}}{\frac{1}{d}}$. Considering the negation operator as the counterpart for logical proportions of the inverse for geometrical proportion, the formal expression of the above property writes:

$$
T(a, b, c, d) \rightarrow T(\bar{a}, \bar{b}, \bar{c}, \bar{d})
$$

i.e., what we have called "code independency". Another reason to look for this property is to note that a numerical proportion $\frac{a}{b}=\frac{c}{d}$ holds independently of the base used for encoding numbers. Therefore, it seems natural to expect the logical proportions behavior to be independent of the way we encode items in terms of the truth or the falsity of properties. For instance $a \wedge \bar{b}$ represents what is specific to $a$ w.r.t. $b$, without any consideration about the way we represent the truth and the falsity. As a consequence, the formula defining a proportion should be valid when we switch 0 to 1 and 1 to 0 in the encoding of a valuation. Which is, as above, exactly translated into

$$
T(a, b, c, d) \rightarrow T(\bar{a}, \bar{b}, \bar{c}, \bar{d})
$$

4. In the numerical case, $\frac{a}{b}=\frac{c}{d}$ and $\frac{c}{d}=\frac{e}{f}$ entails $\frac{a}{b}=\frac{e}{f}$. For a logical proportion $T$, this formally translates into the transitivity property:

$$
T(a, b, c, d) \wedge T(c, d, e, f) \rightarrow T(a, b, e, f)
$$

5. Finally, when dealing with geometrical proportions, we have the following trivial proportions:

- $\frac{a}{a}=\frac{a}{a}$ whose Boolean counterpart is $T(a, a, a, a)$ (full identity),

- $\frac{a}{b}=\frac{a}{b}$ whose Boolean counterpart is $T(a, b, a, b)$ (reflexivity),

- $\frac{a}{a}=\frac{b}{b}$ whose Boolean counterpart is $T(a, a, b, b)$ (sameness),

- $\frac{a}{b}=\frac{\frac{1}{b}}{\frac{1}{a}}$ whose Boolean counterpart is $T(a, b, \bar{b}, \bar{a})$ (exchange-mirroring). 
We have investigated in details the set of the 120 logical proportions to identify the ones satisfying some of the properties above. It appears that only one logical proportion satisfies all the above requirements, namely the analogical proportion $A$. Regarding the other proportions, Table 6 highlights the fact that the homogeneous proportions still behave quite similarly to numerical proportions.

\section{Back to homogeneous proportions}

Proposition 11 has made clear how remarkable are proportions $A, R, P, I$; see also [65]. Indeed they are the only four logical proportions to enjoy two key properties: symmetry (the ordering between pairs $(a, b)$ and $(c, d)$ does not matter in the comparison), and code independency (the truth value of the proportion remains unchanged with a positive or a negative encoding). In this section, we study the links between $A, R, P$, and $I$, some additional properties, and we investigate the structures of opposition inside these proportions.

Starting with a commonality / specificity analysis of a pair of objects, we first retrieve the classical postulate-based view of analogical proportions, together with two other related systems of postulates. It turns out that the logical proportions $A, R, P$ introduced in the previous sections are the only Boolean models (up to logical equivalence) satisfying these respective systems. We close the subsection with a geometrical view of proportions $A, R, P$, which emphasizes their close relationship.

We shall see in the rest of the section how $I$ is related to these proportions through another system of postulates involving negation.

\subsection{Postulates for analogical proportion and two related proportions}

In the following, we show by a simple analysis first suggested in [60] that an analogical proportion-like statement $a$ is to $b$ as $c$ is to $d$, should still hold when permuting the central items $b$ and $c$. Moreover, this analysis also reveals the existence of two other sister proportions. Note that in this subsection we continue to use lowercase letters, $a, b, c, d$ although it may refer to items described by vectors of Boolean variables rather than to a unique Boolean variable.

An analogical statement $a$ is to $b$ as $c$ is to $d$ puts in parallel the pair $(a, b)$, and the pair $(c, d)$. This leads to consider what is common to $a$ and $b$ (let us denote it $\operatorname{com}(a, b)$ ), and what is specific to $a$ and not shared by $b$ (we denote it $\operatorname{spec}(a, b))$. So $\operatorname{com}(a, b)$ can be seen as a set of binary features shared by $a$ and $b$, while $\operatorname{spec}(a, b)$ is a set of binary features possessed by $a$ and not by $b$. Clearly, $\operatorname{com}(a, b)$ and $\operatorname{spec}(a, b)$ are then disjoint subsets. Clearly $\operatorname{com}(a, b)=\operatorname{com}(b, a)$ and generally, $\operatorname{spec}(a, b) \neq \operatorname{spec}(b, a)$. With this view, each item can be represented through its comparison with another one. Thus,

- $a$ is represented by the pair $(\operatorname{com}(a, b), \operatorname{spec}(a, b))$,

- $b$ by the pair $(\operatorname{com}(a, b), \operatorname{spec}(b, a))$,

- $c$ by the pair $(\operatorname{com}(c, d), \operatorname{spec}(c, d))$, 
- $d$ by the pair $(\operatorname{com}(c, d), \operatorname{spec}(d, c))$.

We notice that when going from $a$ to $b$ (resp. from $c$ to $d$ ), $\operatorname{spec}(a, b)$ is changed into $\operatorname{spec}(b, a)(\operatorname{resp} . \operatorname{spec}(c, d)$ is changed into $\operatorname{spec}(d, c))$, while $\operatorname{com}(a, b)$ and $\operatorname{com}(c, d)$ are the respective common parts of the pairs $(a, b)$ and $(c, d)$. An analogical statement such as $a$ is to $b$ as $c$ is to $d$ amounts to compare the pairs $(a, b)$ and $(c, d)$. This can be done in terms of what is common, or in terms of what is specific. Namely, one may state that

- the way $a$ and $b$ differ is the same as the way $c$ and $d$ differ, namely

$$
\operatorname{spec}(a, b)=\operatorname{spec}(c, d) \text { and } \operatorname{spec}(b, a)=\operatorname{spec}(d, c)(1)
$$

- the way $a$ and $b$ differ is the same as the way $d$ and $c$ differ, namely

$$
\operatorname{spec}(a, b)=\operatorname{spec}(d, c) \text { and } \operatorname{spec}(b, a)=\operatorname{spec}(c, d)(2)
$$

- $a$ and $b$ are similar in the same as the way as $c$ and $d$ are similar, namely

$$
\operatorname{com}(a, b)=\operatorname{com}(c, d)(3)
$$

Note that the three options preserve symmetry: Comparing $a, b$ and $c, d$, or comparing $c, d$ and $a, b$ are equivalent.

Let us consider first the statement (1). If we compare $a$ represented as $(\operatorname{com}(a, b), \operatorname{spec}(a, b))$ to $c$ represented as $(\operatorname{com}(c, d), \operatorname{spec}(c, d))$, it appears due to statement (1) that their common part $\operatorname{com}(a, c)$ includes $\operatorname{spec}(a, b)=\operatorname{spec}(c, d)$, while $\operatorname{com}(a, b)$ is changed into $\operatorname{com}(c, d)$ when going from $a$ to $c$. Note that $\operatorname{com}(a, b)$ and $\operatorname{com}(c, d)$ may have a non empty common part com $(a, b, c, d)$. Thus, in set terms, $\operatorname{com}(a, c)=\operatorname{spec}(a, b) \cup \operatorname{com}(a, b, c, d)$ and $\operatorname{spec}(a, c)=\operatorname{com}(a, b) \backslash$ $\operatorname{com}(a, b, c, d)$. We also have $\operatorname{spec}(c, a)=\operatorname{com}(c, d) \backslash \operatorname{com}(a, b, c, d)$. Similarly, when comparing $b$ and $d$, the common part is $\operatorname{spec}(b, a) \cup \operatorname{com}(a, b, c, d)=\operatorname{spec}(d, c) \cup$ $\operatorname{com}(a, b, c, d)$ due to (1) and $\operatorname{com}(a, b)$ is again changed into $\operatorname{com}(c, d)$ when going from $b$ to $d$ (i.e. $\operatorname{com}(a, b) \backslash \operatorname{com}(a, b, c, d)=\operatorname{spec}(b, d)$, and $\operatorname{com}(c, d) \backslash$ $\operatorname{com}(a, b, c, d)=\operatorname{spec}(d, b))$. This amounts to write $\operatorname{spec}(a, c)=\operatorname{spec}(b, d)$, and $\operatorname{spec}(c, a)=\operatorname{spec}(d, b)$. This is exactly statement (1) where $b$ and $c$ are exchanged.

Thus choosing (1), we have retrieved the central permutation postulate that most authors (see, e.g., [17]) associate with analogical proportion (together with the symmetry already mentioned). To avoid any confusion with the previous Boolean model of the analogy proportion, we stick to the traditional notation $a: b:: c: d$ (as recalled in the introduction). This leads to the following postulates:

- $a: b:: a: b$ (and $a: a:: b: b$ ) hold (reflexivity).

- if $a: b:: c: d$ holds then $a: c:: b: d$ should hold (central permutation)

- if $a: b:: c: d$ holds then $c: d:: a: b$ should hold (symmetry).

Remember that the logical proportion $A$ satisfies the three above postulates. Since, as seen in the proof of Proposition 8, reflexivity and central permutation lead to the truth table of the analogical proportion, analogical proportion is the unique Boolean formula (up to equivalence) satisfying the three above postulates. Note that symmetry applies here to the comparison between two pairs of items $(a, b)$ 
and $(c, d)$, but not internally since $a: b$ cannot be replaced with $b: a$. Indeed $a: b:: b: a$ cannot hold since $\operatorname{spec}(a, b) \neq \operatorname{spec}(b, a)$ in general. Such a postulate is more in the spirit of option (2) that we consider now.

Still focusing on specificities, we can consider a new proportion denoted with "!" where $a ! b \quad:: c ! d$ holds as soon as $\operatorname{spec}(a, b)=\operatorname{spec}(d, c)$ and $\operatorname{spec}(b, a)=$ $\operatorname{spec}(c, d)$. It expresses the reverse analogy $a$ is to $b$ as $d$ is to $c$, which obeys the postulates (as can be checked):

- $a ! b \quad:: b$ ! $a$ (and $a ! a \quad:: \quad b ! b)$ should hold (reverse reflexivity)

- if $a ! b \quad:: c ! d$ holds then $c ! b \quad: \quad a ! d$ should hold (odd permutation)

- if $a ! b:: c ! d$ holds then $c ! d:: a ! b$ should hold (symmetry).

Except if $a=b, a ! b \quad: \quad a ! b$ does not hold in general. Having investigated all the possibilities from the viewpoint of specificities, it seems natural to focus on the shared properties, which leads to introduce a new kind of proportion denoted with ";". Then, we have no other choice than defining $a ; b:: c ; d$ as $\operatorname{com}(a, b)=$ $\operatorname{com}(c, d)$. We decide to call this new proportion paralogical proportion. It states that what $a$ and $b$ have in common, $c$ and $d$ have it also. Obviously, this proportion does not satisfy the permutation properties of its two sister relations, but rather:

- $a ; b:: a ; b$ and $a ; b:: b ; a$ always holds (bi-reflexivity)

- if $a ; b:: c ; d$ holds $b ; a:: c ; d$ should hold (even permutation)

- if $a ; b:: c ; d$ holds then $c ; d:: a ; b$ should hold (symmetry).

In summary, the 3 logical proportions $A, R, P$ separately satisfy a set of 3 essential postulates, as seen above. These postulates, which are first order axioms, can be considered as defining 3 types of relation that we called analogy, reverse analogy and paralogy. It is has to be noticed that $A, R, P$ are the unique Boolean formulas (up to equivalence) satisfying their corresponding set of postulates.

It is quite clear that such relations can be defined on other domains than the Boolean one considered in the previous sections. The link with logical proportions is obvious: analogical (resp. reverse analogical, paralogical) proportion is an analogy (resp. reverse analogy, paralogy) on the Boolean universe. In such a general setting, it is worth noticing that full identity is easily deducible for all relations obeying one of the three above sets of postulates, but this is not the case for transitivity (see [61]). This simply means that the Boolean model of logical proportions that we suggest in this paper is richer than a basic model based on the postulates of analogy.

Starting from the postulates of analogy, it is a simple exercise to prove that the following properties are satisfied by an analogy in general (and then, as a particular case, by the analogical proportion $A$ ):

Proposition 18. $a: b:: c: d \rightarrow a: c:: b: d$

$a: b:: c: d \rightarrow d: b:: c: a$

$a: b:: c: d \rightarrow d: c:: b: a$

$a: b:: c: d \rightarrow c: d:: a: b$

$a: b:: c: d \rightarrow b: d:: a: c$ 


$\begin{array}{cccc}\mathbf{a} & \mathbf{b} & \mathbf{c} & \mathbf{d} \\ a & c & b & d \\ d & b & c & a \\ d & c & b & a \\ c & d & a & b \\ b & d & a & c \\ c & a & d & b \\ b & a & d & c\end{array}\left\|\begin{array}{cccc}\mathbf{b} & \mathbf{a} & \mathbf{c} & \mathbf{d} \\ b & c & a & d \\ d & a & c & b \\ d & c & a & b \\ c & d & b & a \\ a & d & b & c \\ c & b & d & a \\ a & b & d & c\end{array}\right\| \begin{array}{cccc}c & \mathbf{b} & \mathbf{a} & \mathbf{d} \\ c & a & b & d \\ d & b & a & c \\ d & a & b & c \\ a & d & c & b \\ b & d & c & a \\ a & c & d & b \\ b & c & d & a\end{array}$

TABle 7. Permutation classes of analogy

$$
\begin{aligned}
& a: b:: c: d \rightarrow c: a:: d: b \\
& a: b:: c: d \rightarrow b: a:: d: c
\end{aligned}
$$

This means that if $a: b:: c: d$ holds then a total of 8 permutations among 24 hold (including $a: b:: c: d$ ) as analogies.

Let us now consider the 2 following permutations $(a, b, d, c)$ and $(a, d, c, b)$, which do not belong to the previous set of permutations:

Proposition 19. For an analogy, we have:

$$
\begin{aligned}
& a: b:: c: d \nrightarrow a: b:: d: c \\
& a: b:: c: d \nrightarrow a: d:: c: b
\end{aligned}
$$

Proof: A simple way to get it is to consider the Boolean model where the valuation 0101 validates the left side of the first implication but not the right side. The valuation 0011 does the same job for the second implication.

As a consequence, among the 24 combinations of $a, b, c, d$, we then distinguish 3 classes of permutations related to analogy that we exhibit in Table 7 , with representative on top in bold font. If one element $(x, y, z, t)$ of a class is such that $x: y:: z: t$ holds, then a similar analogy holds for each element in the whole class. The previous proposition exhibits the fact that if we have an element of a class being an analogy, there is no way to conclude for the 2 remaining classes.

The following proposition, easily deducible from the postulates, establishes a strong link between analogy, reverse analogy and paralogy:

Proposition 20. $a: b:: c: d \equiv a ! b:: d ! c$ and $a: b:: c: d \equiv a ; d:: c ; b$

Thanks to Proposition 20, we have that if $a: b:: c: d$ holds, then $b ! a:: c ! d$ and $c ; b:: a ; d$ hold. These expressions correspond to the three permutations appearing in the first row of Table 7 . But $b ! c:: a ! d$ (corresponding to row 2 of column 2) does not hold: in that case, it is $b ; c:: a ; d$ that holds. This simply means that we cannot go from one column to the next one through a simple operator in Table 7. The above proposition states the interplay between the three sets of three postulates discussed above. Since $A, R, P$ satisfy reflexivity, 


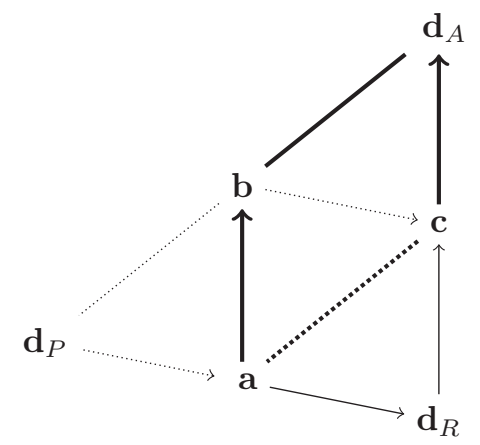

Figure 2. Three parallelograms

symmetry, and respectively central permutation $\left(p_{23}\right)$, odd permutation $\left(p_{13}\right)$, and even permutation $\left(p_{12}\right)$, Proposition 20 can be restated in the Boolean model:

Proposition 21. $A(a, b, c, d) \equiv R(a, b, d, c)$ and $A(a, b, c, d) \equiv P(a, d, c, b)$

Proposition 21 may be also directly derived from the definitions of proportions $A, R, P$ in terms of equivalence between indicators, where their syntactic similarity reflects semantical links that are also visible on their truth tables.

To highlight the differences and the relations between the three proportions $A, R, P$, we may also revisit the "parallelogram metaphor" often used when discussing analogical reasoning in a numerical setting [44, 4, 77], since the parallel between the pairs $(a, b)$ and $(c, d)$ is reminiscent of the equality of two vectors.

It amounts, for instance, to consider $a, b, c$ and $d$ as elements of the real plan $\mathbb{R}^{2}$, and to interpret $a: b:: c: d$ as $\overrightarrow{a b}=\overrightarrow{c d}$. This vectorial equality holds because the coordinates of the 4 points $a, b, c, d$ satisfy an arithmetic analogy: $\forall i \in\{1,2\}, a_{i}-b_{i}=c_{i}-d_{i}$. It simply means that the quadrilateral $a b c d$ is a parallelogram.

In contrast with the Boolean case where an equation $A(a, b, c, x)$ is not always solvable (see Proposition 16), given 3 points $a, b, c$ of the real plan $\mathbb{R}^{2}$, one can always find a point $d$ such that $a b d c$ is a parallelogram (see Figure 2). In fact, from 3 non aligned points, one can build 3 distinct parallelograms; it is the geometrical counterpart of the permutations linking the three proportions $A, R, P$ via Proposition 21. See Figure 2 where the index of $d$ refers to the proportion that generates it from $(a, b, c)$. In Figure 2, we have used different types of lines (with different width, dotted or not, arrows or not) to try to help visualizing the 3 parallelograms.

It is possible to complete the Figure 2 in the following way, in order to introduce the inverse paralogy $I$. Since $I$ can be related to $A$ through the relation $I(a, b, c, d)=A(a, \bar{b}, \bar{c}, d)$, we introduce two points associated to $\bar{b}$ and $\bar{c}$, as the symmetric of $b$ and $c$ with respect to $a . d_{I}$ is then the symmetric 


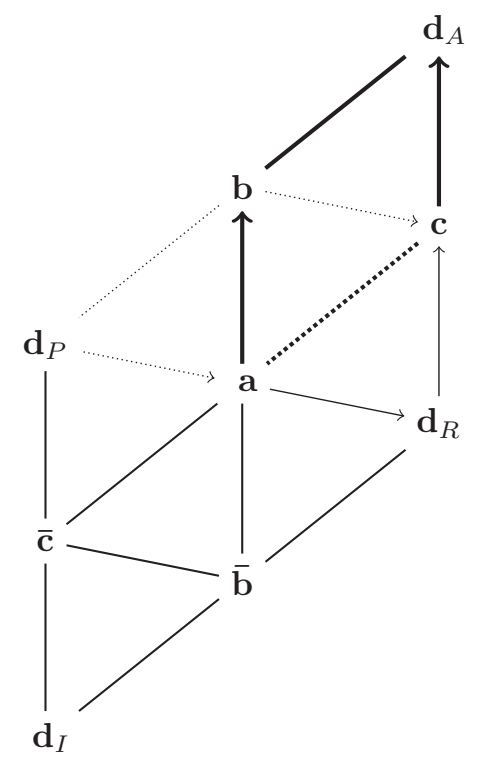

Figure 3. Geometric view of the 4 homogeneous proportions

of $d_{A}$. We thus obtain Figure 3, where many relations can be read such that $I(a, b, c, d)=A(a, \bar{b}, \bar{c}, d)$ (i.e., $d_{I}$ is obtained from $a, \bar{b}$ and $\bar{c}$, as $d_{A}$ from $a, b$ and c), $P(a, b, c, d)=R(a, \bar{b}, \bar{c}, d)$ (which acknowledges the symmetry of $d_{P}$ and $d_{R}$ with respect to $a)$, or $A(\bar{b}, \bar{c}, a, d)=P(a, b, c, d)$ (i.e., $d_{P}$ is obtained by applying to $\bar{b}, \bar{c}$ and $a$ the construction of $d_{A}$ from $a, b$ and $\left.c\right)$, or $A(\bar{c}, a, \bar{b}, d)=R(a, b, c, d)$ (i.e., $d_{P}$ is obtained by applying to $\bar{c}, a$, and $\bar{b}$ the construction of $d_{A}$ from $a, b$ and $c)$, as well as the fact that $A(b, c, \bar{c}, \bar{b})$ holds.

\subsection{Additional properties of homogeneous proportions}

Let us come back to the Boolean setting, where the logical proportions live. We now lay bare some remarkable properties of the proportions $A, R, P, I$. We first provide some equivalent expressions for these proportions, before studying their behavior with respect to conjunction, disjunction and negation, and investigating the power of $A$ as a functionally complete logical operator.

Using the link between proportions $A$ and $P$ expressed by Proposition 21 (and De Morgan laws), we come to a new characterization for analogical proportion which involves the pair $(a, d)$ of the extremes and the pair $(b, c)$ of the means:

$$
A(a, b, c, d) \equiv(a \wedge d \equiv b \wedge c) \wedge(a \vee d \equiv b \vee c)
$$

This property will be considered again when discussing the works of Piaget [57] where this characterization is just taken as the definition of a logical proportion 
(in Piaget's sense). Similarly, using Proposition 21 again, one obtains equivalent expressions for $R$ or for $P$, namely:

$$
\begin{aligned}
& R(a, b, c, d) \equiv(a \wedge c \equiv b \wedge d) \wedge(a \vee c \equiv b \vee d) \\
& P(a, b, c, d) \equiv(a \wedge b \equiv c \wedge d) \wedge(a \vee b \equiv c \vee d)
\end{aligned}
$$

The above characterizations of proportions $A, R, P$, involving only conjunction and disjunction (without negation) make the investigation of their behavior w.r.t. these Boolean operators easier. We only consider the case of $A$ below, for which it is easy to establish the following proposition:

\section{Proposition 22.}

$$
\begin{gathered}
A(a \vee b, a, b, a \wedge b) \text { and } A(a, a \vee b, a \wedge b, b) \\
A(a, b, c, d) \rightarrow A(a \vee e, b \vee e, c \vee e, d \vee e) \\
A(a, b, c, d) \rightarrow A(a \wedge e, b \wedge e, c \wedge e, d \wedge e)
\end{gathered}
$$

The two last statements express a form of compatibility of the analogical proportion with respect to the disjunction or conjunction with a Boolean constant. The first expression shows that any Boolean pair $(a, b)$ is in analogy with its meet and its join. Thanks to Proposition 21, counterparts of these properties can be established for both $R$ and $P$. Regarding the inverse paralogy $I$, this proportion is not directly linked to the previous ones through a permutation. However, a link can be established through the use of the negation operator.

The behavior of $A, R, P, I$ proportions with respect to negation is summarized in Table 8 which is easily derived from their definitions. The third row of this table simply highlights the code independency property. In terms of negation,

\begin{tabular}{l|l}
\hline \multicolumn{1}{c|}{ Analogy } & Reverse analogy \\
$A(a, b, \bar{b}, \bar{a})$ & $R(a, b, \bar{a}, \bar{b})$ \\
$A(a, \bar{b}, b, \bar{a})$ & $R(a, \bar{b}, \bar{a}, b)$ \\
$A(a, b, c, d) \rightarrow A(\bar{a}, \bar{b}, \bar{c}, \bar{d})$ & $R(a, b, c, d) \rightarrow R(\bar{a}, \bar{b}, \bar{c}, \bar{d})$ \\
\hline $\begin{array}{l}\text { Paralogy } \\
P(a, \bar{a}, \bar{b}, b)\end{array}$ & Inverse paralogy \\
$P(a, \bar{a}, b, \bar{b})$ & $I(a, b, \bar{b}, \bar{a}), I(a, b, \bar{a}, \bar{b}), I(a, \bar{a}, \bar{b}, b)$ \\
$P(a, b, c, d) \rightarrow P(\bar{a}, \bar{b}, \bar{c}, \bar{d})$ & $I(a, \bar{b}, b, \bar{a}), I(a, \bar{b}, \bar{a}, b), I(a, \bar{a}, b, \bar{b})$ \\
\hline
\end{tabular}

TABle 8. Behavior of $A, R, P, I$ wrt negation

the link between $A, R, P, I$ proportions is as follows:

Proposition 23. $A(a, b, c, d) \equiv R(a, b, \bar{c}, \bar{d})$, $A(a, b, c, d) \equiv P(a, \bar{b}, c, \bar{d})$, $A(a, b, c, d) \equiv I(a, \bar{b}, \bar{c}, d)$ 
Proof: This is an immediate consequence of our definitions: for instance, starting from the definition of $A(a, b, c, d), a \wedge \bar{b} \equiv c \wedge \bar{d}$ and $\bar{a} \wedge b \equiv \bar{c} \wedge d$, negating $b$ and $d$ gives $a \wedge b \equiv c \wedge d$ and $\bar{a} \wedge \bar{b} \equiv \bar{c} \wedge \bar{d}$, which is exactly the definition of $P(a, b, c, d)$.

It is remarkable that $I$ cannot be linked to the other proportions without the use of negation: this emphasizes the fact that, among the 4 homogeneous logical proportions, $I$ plays a particular role. In fact, a general inverse paralogy could also be defined via a set of postulates. We have diverse options. For instance, using the symbol ? to denote this proportion:

- $\neg(a ? b \quad:: a ? b)$ should hold (non reflexivity) ${ }^{9}$

- if $a ? b:: c ? d$ holds then $a ? c:: \quad b ? d$ should hold (central permutation)

- if $a ? b:: c$ ? $d$ holds then $c$ ? $d:: a$ ? $b$ should hold (symmetry).

or even

- $\neg(a ? a \quad:: \quad b$ ? b) should hold (non sameness)

- if $a ? b:: \quad c ? d$ holds then $b$ ? $a:: c ? d$ should hold (even permutation)

- if $a ? b:: c ? d$ holds then $c ? d:: a ? b$ should hold (symmetry).

As a consequence, we have the following result:

Proposition 24. $I$ is the unique Boolean formula (up to equivalence) satisfying these 2 sets of postulates.

Proof: See Annex B.

However, we may also notice that if we consider the following other set of postulates for instance, which is the set of postulates related to Reverse Analogy $R$ but negating the first postulate:

- $\neg(a ? b \quad:: \quad b$ ? $a)$ should hold (non reverse reflexivity)

- if $a ? b:: c$ ? $d$ holds then $c ? b \quad:: a$ ? $d$ should hold (odd permutation)

- if $a ? b:: c$ ? $d$ holds then $c$ ? $d:: a$ ? b should hold (symmetry),

$I$ is not the unique proportion that satisfies this set of postulates in the Boolean model. For instance, the conditional proportion $a|b:: c| d$ satisfies the above postulates.

Another natural question is to wonder if each of the logical proportions $A, R, P, I$ is functionally complete. For instance, let us see if we recover all the Boolean connectives by using the analogical proportion only. It is quite straightforward to check the following equivalences:

- $1 \equiv A(1,1,1,1)$, or more generally $1 \equiv A(a, a, a, a)$,

- $0 \equiv A(0,0,0,1)$ or more generally $0 \equiv A(0, a, a, 1)$,

- $a \equiv A(a, 1,1,1)$ and $\neg a \equiv A(a, 0,0,0)$,

- $a \wedge b \equiv A(1, a, b, 1)$ and $\neg a \wedge \neg b \equiv A(0, a, b, 0)$,

${ }^{9}$ It has to be noticed that $I$, viewed as a binary relation between pairs is not irreflexive $(S$ is irreflexive iff $\forall x, \neg S(x, x))$ in the classical sense, as 0101 and 1010 are valid valuations for $I$. 
- $a \wedge \neg b \equiv A(1, a, b, 0)$ and $\neg a \wedge b \equiv A(0, a, b, 1)$,

- $(a \equiv b) \equiv A(a, b, 1,1)$,

- $\neg(a \equiv b) \equiv A(a, 0,1, b)$ (i.e., the exclusive or connective).

Due to the previous equivalences, it appears that the analogical proportion $A$, considered as a quaternary connector, is functionally complete since we can express negation and conjunction as a single analogical proportion. Similar results hold for the 3 other homogeneous proportions; we have, e.g. $\neg a \equiv I(a, 1,1,0)$ and $a \wedge b \equiv I(a, 0,0, b)($ since $a \wedge b \equiv A(1, a, b, 1) \equiv A(a, 1,1, b) \equiv I(a, 0,0, b))$.

Starting from $A(a, a \vee b, a \wedge b, b)$, using the permutation property of analogical proportion, we also see that:

- $a \equiv A(1, a \vee b, a \wedge b, b)$ since $A(1, a \vee b, a \wedge b, b)$ only holds when $a$ is true, whatever the truth value of $b$. Similarly, $b \equiv A(a, a \vee b, a \wedge b, 1)$,

- $a \vee b \equiv A(a, 1, a \wedge b, b)$ (similar reasoning),

- $a \wedge b \equiv A(a, a \vee b, 1, b)$ (similar reasoning).

Nevertheless, the disjunction $a \vee b$ cannot be defined via a unique analogical proportion involving only $a, b, 0$ and 1 . The reason is that a valuation $\sigma$ validates a proportion $A(a, b, c, d)$ only when there is an even number of variables having the truth value 1 . But the 3 valuations leading $(a, b)$ to $(1,1)$ or $(1,0)$ or $(0,1)$ validate $a \vee b$, which implies that we should have at least one valuation with an odd number of 1 validating $A(a, b, c, d)$ in order to have an equivalence. This reasoning applies to all the other proportions $R, P, I$ and shows that it is not possible to define the $\checkmark$ connective using only one of the homogeneous proportion among $R, P$ or $I$.

\subsection{Homogeneous proportions and Piaget's group of transformations}

In his studies about the principles of knowledge acquisition by a human being, the psychologist Jean Piaget [56] investigated basic transformations of statements which are at work in human language. He identified a group of 4 transformations, denoted $\mathcal{I}, \mathcal{N}, \mathcal{R}, \mathcal{C}$ which operate on Boolean logical formulas of the form $\phi(p, q, r, \ldots)$. They are defined in the following way:

- the identity $\mathcal{I}: \mathcal{I}(\phi(p, q, r, \ldots))=\phi(p, q, r, \ldots)$

- the negation or inverse $\mathcal{N}: \mathcal{N}(\phi(p, q, r, \ldots))=\neg \phi(p, q, r, \ldots)$

- the reciprocation $\mathcal{R}: \mathcal{R}(\phi(p, q, r, \ldots))=\phi(\neg p, \neg q, \neg r, \ldots)$

- the correlation $\mathcal{C}: \mathcal{C}(\phi(p, q, r, \ldots))=\neg \phi(\neg p, \neg q, \neg r, \ldots)$

Obviously, these transformations are not independent and we can note that $\mathcal{I}=$ $\mathcal{N} \circ \mathcal{R} \circ \mathcal{C}, \mathcal{N}=\mathcal{C} \circ \mathcal{R}=\mathcal{R} \circ \mathcal{C} \ldots$ Basically $\{\mathcal{I}, \mathcal{N}, \mathcal{R}, \mathcal{C}\}$ is a commutative group w.r.t. the composition of operators (denoted $\circ$ ).

Let us first notice that the code independency property directly involves Piaget's reciprocation, i.e., $T(a, b, c, d) \rightarrow \mathcal{R}(T(a, b, c, d))$. More importantly, homogeneous proportions behave nicely with respect to Piaget's transformations. Indeed the following easy result holds for the analogical proportion.

\section{Proposition 25.}

$$
\forall \phi, \psi, A(\mathcal{I}(\phi), \mathcal{C}(\psi), \mathcal{R}(\psi), \mathcal{N}(\phi))
$$


Proof: Indeed the truth value of $\mathcal{I}(\phi)$ and $\mathcal{N}(\phi)$ are opposite whatever $\phi$, the same is true with $\mathcal{R}(\psi)$ and $\mathcal{C}(\psi)$ whatever $\psi$. So this proportion necessarily obeys one of the four valid analogical patterns 1100, 1010,0011,0101.

Moreover, thanks to Propositions 21 (for $R$ and $P$ ) and 23 (for $I$ ), we can recover similar properties for $R, P, I$, namely

$$
\begin{aligned}
& \forall \phi, \psi, R(\mathcal{I}(\phi), \mathcal{C}(\psi), \mathcal{N}(\phi), \mathcal{R}(\psi)), \\
& \forall \phi, \psi, P(\mathcal{I}(\phi), \mathcal{N}(\phi), \mathcal{R}(\psi), \mathcal{C}(\psi)), \\
& \forall \phi, \psi, I(\mathcal{I}(\phi), \mathcal{R}(\psi), \mathcal{C}(\psi), \mathcal{N}(\phi)) .
\end{aligned}
$$

\subsection{Structures of opposition among proportions $A, R, P$ and $I$}

The construction of the logical proportions rely on the interplay of two similarity indicators and two dissimilarity indicators. When this interplay is "homogeneous" with respect to the pairs of binary variables considered, we obtain the 4 homogeneous proportions, as explained in this paper. The fact that there are 4 indicators, and then 4 homogeneous proportions, suggests to organize them into squares in order to lay bare and visualize the forms of oppositions that exist between them. In some sense, this fits with the tradition of Aristotelian logic where a so-called square of opposition was introduced at the basis of syllogistic reasoning. The Aristotelian square involves four logically related statements exhibiting universal or existential quantifications ("every $x$ is $p$ ", "no $x$ is $p$ ", "some $x$ is $p$ ", "some $x$ is not $p "$ ), where two negations are at work. However, the structure of oppositions in the square of indicators and in the square of homogeneous proportions differs from the one existing in the Aristotelian square, as we shall see. In the second part of this subsection, we shall use an hexagonal extension of the Aristotelian square for some further analysis of the relations between logical operators related to the ideas of similarity/dissimilarity and the analogical/paralogical proportions [67].

\subsubsection{A square of opposition for similarity and dissimilarity.}

As made clear in the previous sections, the formalization of the analogical proportion and the other logical proportions leads to introduce the concept of indicators. In logical terms, similarity corresponds to the indicators $S_{a, b} \triangleq a \wedge b$ and $S_{a, b}^{\prime} \triangleq \bar{a} \wedge \bar{b}$, while dissimilarity corresponds to indicators $D_{a, b} \triangleq a \wedge \bar{b}$ and $D_{a, b}^{\prime} \triangleq \bar{a} \wedge b$. These four expressions can be easily arranged into an elementary square of opposition, where one moves horizontally (resp. vertically) by negating the first logical variable, i.e. $a$ (resp. the second logical variable, i.e. $b$ ). The expressions that are related by diagonals are exchanged by negating each variable. See Figure 4 . Note that the 4 vertices of the square correspond here to mutually exclusive situations, which is not the case in the Aristolelian square.

Following the same principle, the four homogeneous proportions can be organized into a similar square. See Figure 5. Indeed, analogy $\mathbf{A}$ defined by $\left(D_{a, b} \equiv D_{c, d}\right) \wedge$ $\left(D_{a, b}^{\prime} \equiv D_{c, d}^{\prime}\right)$, (corresponding to the logical expression $\left.(a \wedge \bar{b} \equiv c \wedge \bar{d}) \wedge(\bar{a} \wedge b \equiv \bar{c} \wedge d)\right)$ and paralogy $\mathbf{P}$ defined by $\left(S_{a, b} \equiv S_{c, d}\right) \wedge\left(S_{a, b}^{\prime} \equiv S_{c, d}^{\prime}\right)$ (corresponding to the 


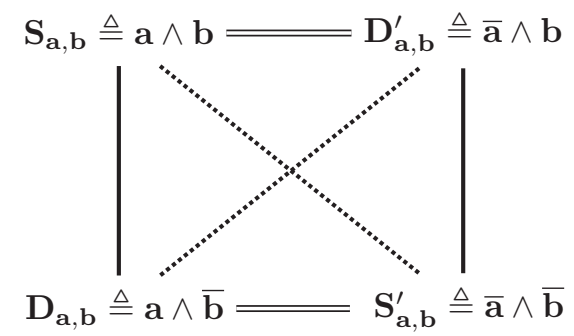

Figure 4. Square of indicators

logical expression $(a \wedge b \equiv c \wedge d) \wedge(\bar{a} \wedge \bar{b} \equiv \bar{c} \wedge \bar{d}))$ are exchanged horizontally by negating the second and the fourth (or the first and the third) logical variable. Reverse analogy $\mathbf{R}$ defined by $\left(D_{a, b} \equiv D_{c, d}^{\prime}\right) \wedge\left(D_{a, b}^{\prime} \equiv D_{c, d}\right)$ (corresponding to the logical expression $(\bar{a} \wedge b \equiv c \wedge \bar{d}) \wedge(a \wedge \bar{b} \equiv \bar{c} \wedge d))$ and inverse paralogy I defined by $\left(S_{a, b} \equiv S_{c, d}^{\prime}\right) \wedge\left(S_{a, b}^{\prime} \equiv S_{c, d}\right)$ (corresponding to the logical expression $(a \wedge b \equiv \bar{c} \wedge \bar{d}) \wedge(\bar{a} \wedge \bar{b} \equiv c \wedge d))$ are exchanged in the same way. $\mathbf{P}$ and $\mathbf{R}$ are exchanged vertically by negating the first and the fourth (or the second and the third) logical variable, and it is the same for $\mathbf{A}$ and $\mathbf{I}$. Moreover $\mathbf{A}$ and $\mathbf{R}$ one the one hand, and $\mathbf{P}$ and $\mathbf{I}$ on the other hand are nicely opposed through diagonals. Again the four vertices of the square correspond to mutually exclusive situations. Note also that the names of the vertices here, $\mathbf{P}, \mathbf{A}, \mathbf{I}$ and $\mathbf{R}$, should not be confused with the traditional names of the vertices of the Aristotelian square, i.e., A, E, $\mathbf{O}$ and $\mathbf{I}$.

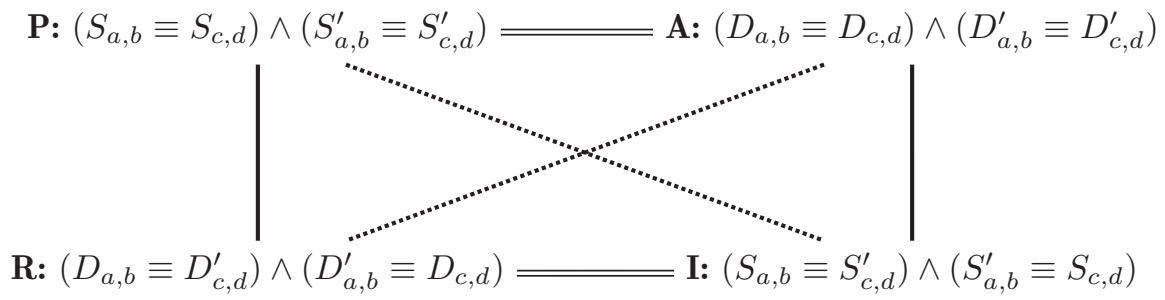

FiguRE 5. Square of homogeneous logical proportions

It would be possible to organize into squares other logical proportions, in particular the 16 conditional proportions, but this is beyond the scope of this paper.

\subsubsection{Analogical and paralogical proportions in hexagons of opposition.}


We first provide a brief reminder about the hexagonal extension of the Aristotelian square, before applying it to homogeneous proportions. It has been noticed since Aristotle that a statement (A) of the form "every $x$ is $p$ " is negated by the statement $(\mathrm{O})$ "some $x$ is not $p$ ", while a statement like $(\mathrm{E})$ "no $x$ is $p$ " is clearly in even stronger opposition to the first statement (A). These three statements, together with the negation of the last statement, namely (I) "some $x$ is $p$ ", give birth to the Aristotelian square of opposition in terms of quantifiers $A: \forall x p(x), E: \forall x \neg p(x)$, $I: \exists x p(x), O: \exists x \neg p(x)$, pictured in Figure 6. Such a square is usually denoted by the letters A, I (affirmative half) and E, O (negative half). The names of the vertices come from a traditional Latin reading: AffIrmo, $\mathrm{n} \mathbf{E g} \mathbf{O})$. Another standard example of the square of opposition is in terms of modalities: $A: \square r, E: \square \neg r$, $I: \diamond r, O: \diamond \neg r$ (where $\diamond r \equiv \neg \square \neg r$ ). As can be seen from these two examples, different relations hold between the vertices. Namely,

- (a) $A$ and $O$ are the negation of each other, as well as $E$ and $I$;

- (b) $A$ entails $I$, and $E$ entails $O$;

- (c) $A$ and $E$ cannot be true together, but may be false together, while

- (d) $I$ and $O$ cannot be false together, but may be true together.

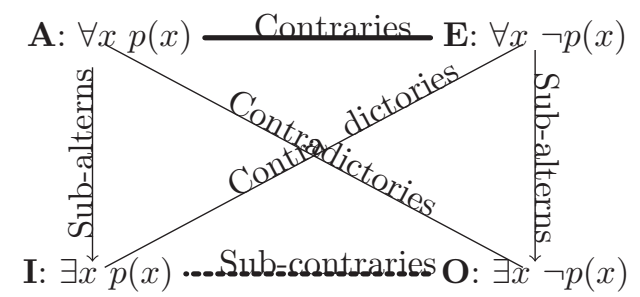

Figure 6. Square of opposition

As proposed and advocated by Blanché [7, 8], it is always possible to complete a square of opposition into a hexagon by adding the vertices $Y={ }_{\text {def }} I \wedge O$, and $U={ }_{\text {def }} A \vee E$. It fully exhibits the logical relations inside a structure of oppositions generated by the three mutually exclusive situations $A, E$, and $Y$, where two vertices linked by a diagonal are contradictories, $A$ and $E$ entail $U$, while $Y$ entails both $I$ and $O$. Moreover $I=A \vee Y$ and $O=E \vee Y$. The interest of this hexagonal construct has been especially advocated by Béziau [5] in the recent years for solving delicate questions in paraconsistent logic modeling. Conversely, three mutually exclusive situations playing the roles of $A, E$, and $Y$ always give birth to a hexagon [20], which is made of three squares of opposition: $A E O I, A Y O U$, and EYIU. See Figure 7.

We are going now to apply the fact that a tri-partition leads to a hexagon of opposition, by considering tri-partitions of sets of 4-tuples of binary valuations. It is clear that there is a unique quaternary connective that makes true a particular subset of 


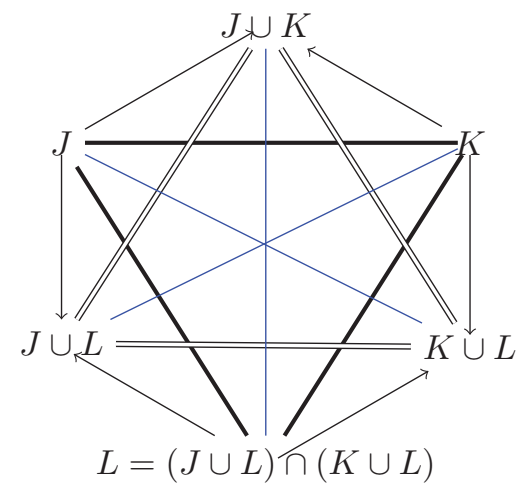

Figure 7. Hexagon induced by a tri-partition $(J, K, L)$

4-tuples of binary valuations, and which is false on all the other subsets of 4-tuples of valuations. We start by considering the whole set of the 16 possible 4 -tuples of binary valuations. The partition that we are going to consider is the following one $J=\{0110,1001)\}, K=\{0001,0010,0100,1000,1110,1101,1011,0111)\}$, $L=\{0000,1111,1010,0101,1100,0011)\}$. As can be seen, in this partition of the 16 possible 4 -tuples of valuations into 3 mutually exclusive subsets, $L$ corresponds to the truth table of the analogy $A, K$ gathers the 8 patterns including an odd number of ' 1 ' (and thus of ' 0 ') , while $J$ gathers the two characteristic patterns 0110 and 1001 of the reverse analogy. The corresponding hexagon is pictured in Figure 8. This hexagon has a nice interpretation in terms of analogical dissimilarity in the sense of [50]. Indeed the analogical dissimilarity of the 6 valuation patterns in $L$ is 0 , since they correspond to the 6 cases where $A$ holds true; the analogical dissimilarity of the 8 valuation patterns in $K$ is 1 , since in each case it is enough to switch one bit for getting a pattern for which proportion $A$ is true; the analogical dissimilarity of the 2 patterns in $J$ is 2 since one needs to change 2 bits to get a pattern where proportion $A$ is true. It is also noticeable that the set of patterns in $J \cup L$ corresponds to the truth table of a quaternary connective that exactly corresponds to S. Klein's view of analogy [37, 38], defined as:

$$
\operatorname{Klein}(a, b, c, d) \triangleq(a \equiv b) \equiv(c \equiv d)^{10},
$$

which is closely related to the equation solving problem for proportions $A, R$, $P$, and which we further comment in the related work section. The vertex corresponding to $K \cup L$, named "approximate similarity" in Figure 8, is associated with an operator which is true in the cases where the analogical dissimilarity is

\footnotetext{
${ }^{10}$ As indicated by the hexagonal structure of Figure $8, \quad A(a, b, c, d)$ logically entails $\operatorname{Klein}(a, b, c, d)$. It is also worth noticing that $A(a, b, c, d)$ has two remarkable implicants, namely $(a \equiv b) \wedge(c \equiv d)$ and $(a \equiv c) \wedge(b \equiv d)$ which are true for only 4 valuation patterns. Similar logical bracketing can be obtained for the three other homogenous proportions.
} 
not maximal. The hexagon of Figure 8 is clearly associated with the analogical proportion $A$. There are 3 other similar hexagons associated with each of the 3 other homogeneous proportions, changing $L$ and $J$ in the appropriate way.

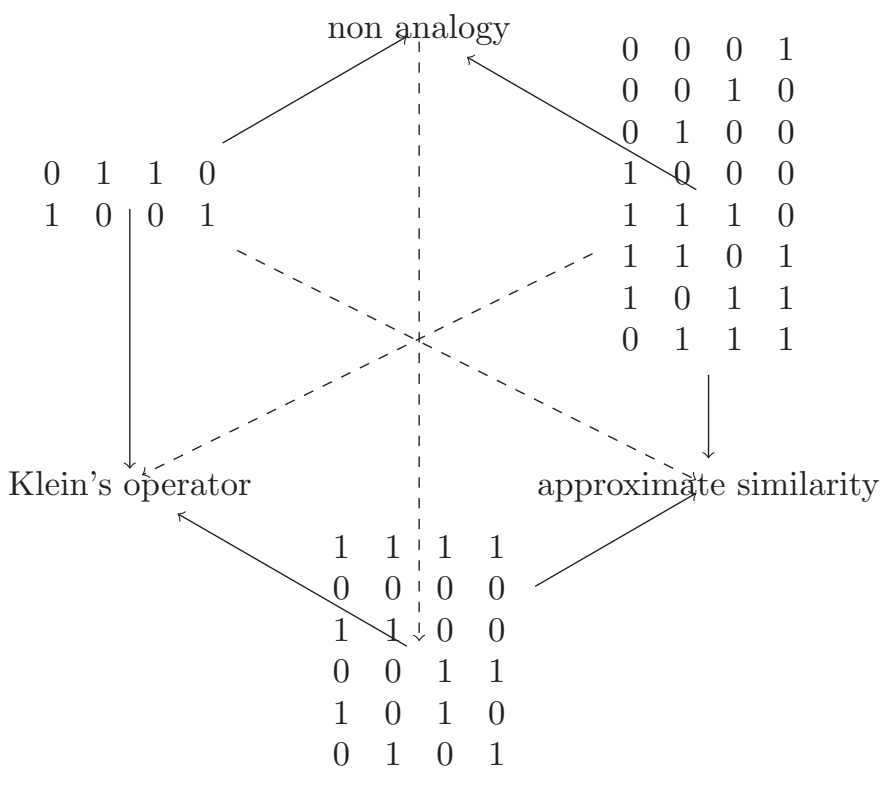

Figure 8. Hexagon induced by analogical dissimilarity

When choosing a tri-partition, it is not compulsory to consider all the 16 valuation 4-tuples. One may, for instance, restrict ourselves to the patterns that make the analogical proportion, or the paralogical proportion true. Then, as can be seen in Figure 9, we get interesting results since one may provide a logical semantic counterpart to two hexagons respectively hinted by Moretti [53] and by Béziau [6] recently. Note that in the second hexagon "analogy" is understood in the strong sense of the restriction of $A$ to its characteristic pattern, while "opposition" corresponds to the restriction of $R$ to its characteristic pattern. "Contrariety" in the first hexagon corresponds to the characteristic pattern of the inverse paralogy $I$. Note also that the notions of "similarity" and "difference" slightly differ in the two hexagons, while the notions of "identity" and "sameness" coincide (and correspond here to the characteristic pattern of paralogy).

This section has surveyed properties that make the 4 homogeneous proportions $A, R, P, I$ remarkable among the 120 logical proportions. Indeed, using different points of view, we have seen that these proportions naturally emerge thanks 

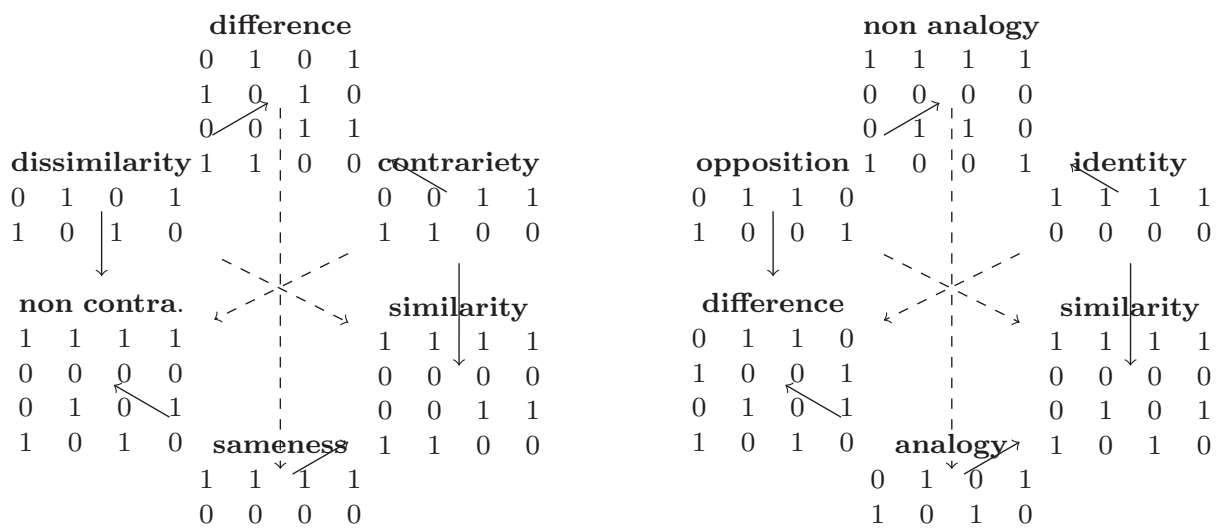

Figure 9. Moretti's and Béziau's hexagons induced by decomposing analogy and paralogy truth tables. The hexagons show the patterns for which the corresponding vertices are true.

to their singular properties. To the best of our knowledge, neither the 120 logical proportions, nor the 4 homogeneous ones have been considered in the literature before (if we except our own recent works). This is why the following related work section only deals with the analogical proportion.

\section{Related work}

As emphasized by many authors, analogical reasoning is central in human intelligence. For instance, the psychologist William James in his 1890 book [35] wrote "A native talent for perceiving analogies is ... the leading fact in genius of every order". Moreover, as said in the introduction, analogical proportion, and more generally analogy, is a very old concept whose interest is shared by diverse scientific communities. In the following, we only concentrate on works focusing on analogical proportion, and which are logically, or at least computationally oriented. We first summarize the pioneering works of [57] and [37] which are, as far as we know, the only ones who contributed to a Boolean view of analogical proportions. Also somewhat close to the framework presented here, are the works of a group of researchers who developed a set-theoretic view of analogical proportion in the last decade, which will be reviewed next. We will then successively consider logical or algebraic approaches differing from the framework presented here, and other psychologically inspired and computationally oriented works.

\subsection{Boolean view of analogical proportions: the works of J. Piaget and S. Klein}

As already said in the introduction, at the occasion of the study of the behavior of his transformation group on ternary connectives [57], J. Piaget introduces a notion logical proportion. Namely, four Boolean formulas $\alpha, \beta, \gamma, \delta$ make a logical proportion iff $\alpha \wedge \delta \equiv \beta \wedge \gamma$ and $\alpha \vee \delta \equiv \beta \vee \gamma$ holds. Moreover, Piaget used the notation 
$\frac{\alpha}{\beta}=\frac{\gamma}{\delta}$ in this situation. As we have previously observed, the conjunction of the two above conditions is strictly equivalent to the definition given for $A(\alpha, \beta, \gamma, \delta)$. On this basis, Piaget [57] provides many valid logical proportions (in his sense), that can be easily translated in known properties of the analogical proportion $A$, for instance, in the notations of this paper, Piaget showed that the following holds

$$
\forall \phi, \psi, \quad A(\mathcal{I}(\phi), \mathcal{R}(\psi), \mathcal{C}(\psi), \mathcal{N}(\phi))
$$

which can be recovered from Proposition 25 using central permutation, or

$$
\forall \phi, \psi, \quad A(\mathcal{I}(\phi), \mathcal{I}(\psi), \mathcal{N}(\psi), \mathcal{N}(\phi))
$$

which is just exchange mirroring. More generally, Piaget gave different instances of Proposition 25, substituting different formulas for $\alpha, \beta, \gamma, \delta$. For instance, with $\phi=(p \wedge q) \vee(p \wedge \bar{q})$ and $\psi=p \vee q$ in the above expression, we get $\mathcal{N}(\phi)=\bar{p}$ and $\mathcal{N}(\psi)=\bar{p} \wedge \bar{q}$ leading to

$$
A((p \wedge q) \vee(p \wedge \bar{q}), p \vee q, \bar{p} \wedge \bar{q}, \bar{p}) .
$$

In fact, all the Piaget's formulas, involving general Boolean formulas can be recovered using the properties of the analogical proportion $A$. This shows once again that the notion of logical proportion is definitely linked to the human cognitive process. But, what is retrospectively amazing, is that Piaget never referred to analogical proportions, when speaking of his logical proportions [57, 58], although he mentioned analogy in some of his previous book [55].

In a series of works, S. Klein [36, 37, 38] has proposed a way of solving analogical proportion-like equations based on a so-called Appositional Transformation Operator or ATO (later named "Analogical Transformation Operators" [39]), denoted $*$, which is nothing but the strong binary equivalence: $* a b$ is just $a \equiv b$. He observed that the repeated use of $*$ allows him to obtain the solution $d=* c(* a b)=c \equiv(a \equiv b)$. We have seen that this is indeed the solution of the analogical proportion equation when the solution exists, but otherwise provides the solution of a paralogical, or a reverse analogical proportion equation. In other words, his constructive view of analogy corresponds to what we called Klein quaternary connective in Section 5.4.2, namely Klein $(a, b, c, d)=(a \equiv b) \equiv(c \equiv d)$, which is less restrictive than the definition given in this paper. With this definition, if " $a$ is to $b$ as $c$ is to $d$ ", then " $b$ is to $a$ as $c$ is to $d$ ", which is hardly acceptable since it does not fit well with the commonsense idea of analogy. In its truth table, Klein connective has two more lines, corresponding to the valuations 0110 and 1001, leading to true than the analogical proportion defined in this paper. Nevertheless, Klein's definition allows him to solve simple verbal and visual analogies, and to explain numerous anthropological patterns appearing in various cultures.

The above-mentioned contributions by Piaget and Klein are the two first works that have some relation with the idea of a logical model for analogical proportion. In the following we review more recent works that propose either a formal modeling of analogy or a computational approach to analogical reasoning. 


\subsection{Other algebraic and logical models}

A formal study of analogical proportions (obeying the three postulates of section 5.1 ) has been first proposed in $[43,44]$ in a computational linguistics perspective, and further developed in [76]. These authors have proposed different set-theoretic definitions of analogical proportions. The main one, due to [76], reads as follows. Let $A, B, C, D$ be subsets of features, the analogical proportion $A: B:: C: D$ holds if and only if there exist four subsets $U, V, W$ and $Z$, such that $A=U \cup V$, $B=U \cup W, C=Z \cup V, D=Z \cup W$. An algebraic, factorization-based, generalization of this definition has been proposed and applied to various structures ranging from semi-groups to lattices, including words over finite alphabets and finite trees $[75,76,51,1]$. One of the main interest of these approaches is the fact that they also lead to effective implementations, generally dedicated to machine learning applications. In particular, the works of $[3,50]$ highlight the fact that the use of analogical proportions as a classification tool, leads to results which outperform standard classification techniques. Besides, the above set-theoretic definition has been later restated in a different, simpler but equivalent way in [52], as $A \backslash B=C \backslash D$ and $B \backslash A=D \backslash C$. Moreover, the logical counterpart of this definition is also studied in [52].

Due to the brittleness of its conclusions, analogical reasoning is not amenable to a formal logic framework in a straightforward manner. Nevertheless, a logical view, quite different from the propositional modeling of analogical proportions discussed here, has been proposed in [15] using a first order logic setting. Considering two particular terms $s$ and $t$ that share a common property $P$ (i.e $P(s)$ and $P(t)$ hold), where moreover $s$ satisfies an additional property $Q$ (i.e. $Q(s)$ holds), then by an "analogical jump" $t$ should satisfy $Q$ (i.e. $Q(t)$ should hold). The legitimacy of this conclusion cannot be insured without some external background knowledge. But, this background knowledge alone should not be sufficient to entail the property $Q(t)$ of the target (without the use of $P(s)$ and $Q(s)$ ). A considerable amount of works has been done to identify the weakest external conditions of this kind making the inference scheme valid. It appears that such external conditions are extremely strong, since they are not far from expressing a functional relation. The reader is referred to [15] for details.

A rather different approach has been developed by [72, 30, 29]. Still logicbased, both higher-order features and anti-unification [59] are added to the classical first order framework, leading to what is known as the Heuristic-Driven Theory Projection (HDTP) framework. In these works, all the items are represented as first order terms, but the definition of analogical proportion is stated in another manner. More precisely, $a: b:: c: d$ holds iff we can find 2 patterns (i.e. terms) $P$ and $Q$ such that $P \sigma_{1}=a, P \sigma_{2}=c$ (i.e. $\sigma_{1}$ and $\sigma_{2}$ denote substitutions and $P$ generalizes $a$ and $c$ ), and $Q \sigma_{1}=b$ and $Q \sigma_{2}=d$ (i.e. $Q$ generalizes $b$ and $d$ ). In other words, the pair $P, Q$ allows to transform $a$ into $b$ and $c$ into $d$ which makes the proportion to hold. $P$ and $Q$ denote properties, which have to be found, thus the need for higher order logic. Instead of using the basic logical equivalence $\equiv$, an 
equational theory $E$ can be added to deal with more general pattern via equality modulo $E$. This suggests an equation-solving process where $d$ is unknown and computed (when such a $d$ exists) via an anti-unification process extracting $P$ and $Q$ from $a, b, c$. Another approach that refers to unification is the one proposed by [14] whose starting point is a general theory of pattern perception. As can be seen, this kind of approaches, as the one of the previous paragraph, does not encode the analogical reasoning mechanism at the same level as with the propositional modeling of analogical proportions.

\subsection{Other computational approaches}

From a more practical viewpoint, analogical reasoning, considered a powerful heuristic device, has been investigated in artificial intelligence for a long time. Evans' Analogy program [22] is the first attempt at implementing an analogysolving program in a setting of a knowledge representation language. It was designed for solving a class of IQ puzzles of the form $A$ is to $B$ as $C$ is to which of $D_{1}$, or $D_{2}$ or $\ldots D_{n}$ ? involving simple geometric figures. The program, starting from a representation of $A$ and $B$, first looks for transformation rules starting from $A$ and leading to $B$, and from $C$ to each $D_{i}$. Then it selects the $D_{i}$ associated with the set of rules that are the most similar to the ones linking $A$ and $B$. The work of [40] follows the same philosophy, but applies it to an analogy-based first-order theorem prover where $A$ is stated as a theorem whose a known proof is $B$ (represented as an ordered set of clauses) and $C$ is a new theorem to be proved. As previously, the system heavily relies on the representation language and more importantly, of the database of available theorems and lemma. Such a use of analogy has been pursued for several researchers; see [49].

In the same spirit, [82] builds up an analogical engine able to establish links between a current situation and previously seen ones, in terms of logical rules. Following this line of thought, the Structure Mapping Theory (SMT) [26], also based on psychological and cognitive concerns [27], is probably the first framework abstracting from first order logic and naturally leading to higher order logic where an analogy is characterized by the mapping of relations between objects, rather than attributes of objects. Despite its abstraction, the model led to what is known as the Structure Mapping Engine (SME) [23] exploring SMT, and providing an effective (in the sense where most of the steps are polynomial) "tool kit" for constructing matching algorithms consistent with SMT. For instance, more recently, SME has been used for analogical comparison of sketches [24], allowing to solve analogies from geometrical sketches, or even Raven's tests as in [48]. It is important to note that the SMT-based engines are generally not constructive in the sense they cannot solve an analogical equation from scratch. They are more designed to choose a solution among a set of candidate solutions, as it was already the case for Evans's program. The Vivomind engine of Sowa [74], implemented on top of a conceptual graphs representation, is also SMT-based, but adds some low-level processes representative of the human brain behavior. [54] combines SMT with an attribute matching process to solve geometric analogy problems. 
The above approaches deal with symbolic representations. Others [25], influenced by connectionism, are more numerically-oriented (which may have some merits by allowing for graded view of similarity). A well-known example of this trend is the Copycat project [33], which focuses on analogical proportions whose experimental domain deals with incomplete analogical proportions of the form $a b c$ is to abd as ijk is to ? The program can lead to several options, using parallel search and weighting the diverse answers, thus relaxing the rigid framework of SMT. Another more recent example of the use of numerical similarity evaluation as a basis for selecting a candidate solution can be found in [41], On a more theoretical side, [12] established a link between analogical reasoning and Kolmogorov complexity used for evaluating the changes from $A$ to $B$, or from $C$ to $D_{i}$. The author advocates a kind of "simplicity principle" to serve as a starting point for modeling analogical proportion via Kolmogorov theory. This approach is in complete line with the works of [11] in which "choose the pattern that provides the briefest representation of the available information" is acknowledged as the rational basis of a wide range of cognitive processes, including analogical reasoning. Besides, a Kolmogorov theory-based quantitative definition of analogical proportions has been proposed in [2].

Obviously, there are still many other kinds of works related to analogy. For instance, it is worth to mention that analogical proportion is not only an amazing object, but also a crucial concept having various instances in natural language leading to extensive investigations, and providing encouraging results for automatic translation as in $[45,46,42,81,9]$ or in text comprehension $[79,78]$, or even in recommendation systems [71].

In this section, we have focused on those works that provide, at least at the algorithmic level, some formal view of the analogical process, generally in relation with the idea of analogical proportions. With respect to all these works, the approach reported here enjoys at least three distinctive features: i) its simplicity, due to an easy-to-understand propositional representation; ii) its coverage, since the analogical proportion is a noticeable element among a large set of logical proportions obeying the same type of similarity/dissimilarity-based pattern; iii) its inference ability, thanks to the equation-solving property.

\section{Conclusion}

Our initial works on this topic $[60,61]$ were centered on the 3 proportions $A, R, P$, then expanded to the 15 proportions satisfying full identity [64]. It has appeared [62] that many other options are available for defining further proportions: we have called these proportions, defined via a pair of equivalences, logical proportions. These equivalences relate the basic similarity and dissimilarity indicators that can be considered when comparing two states of fact. A first inventory of the 120 existing logical proportions was provided both through a syntactic typology, and 
through the study of some meaningful semantical properties. A more dedicated study has recently focused on the 4 homogeneous proportions $A, R, P, I$ [65].

The intended purpose of this paper is to provide a systematic discussion of the idea of logical proportions, and a rigorous study of their relationships and noticeable properties. In that respect, the present paper is not just the structured union of the results that can be found in the previously-cited papers, but includes many new results as well as new perspectives. Nevertheless, logical proportions, which apparently have never been considered before in spite of their conceptual simplicity, have to be further investigated.

In fact, thanks to a software implementation [66], a number of new noticeable, or even in some cases amazing properties have been computationally checked through enumerations. These results, for which we have not yet direct proofs, may be regarded as conjectures. A selection of them can be found in Annex C. Let us mention only here the existence of 4 remarkable semi-hybrid proportions such that each satisfies three of the four following properties $T(a, a, a, \bar{a}), T(a, a, \bar{a}, a)$, $T(a, \bar{a}, a, a), T(\bar{a}, a, a, a)$ (note that satisfying all of them corresponds to the negation of Klein's operator in hexagon of Figure 8 which is true for 8 valuation patterns). These proportions are closely related with the idea of spotting the odd one out, or if we prefer of picking the one that doesn't fit among 4 items [69]. It is worth noticing that the setting of logical proportions both includes symmetrical proportions that compare pairs on a par in a homogeneous way (as with analogy), and non symmetrical ones that correspond to a different cognitive process.

Another worth investigating issue, is the extension of the study of Boolean logical proportions to multiple-valued settings. Even, if some proposal has been made for defining $A, R, P$, in the case of a 3 -valued, and a $[0,1]$-valued setting $[63,68]$ with the idea of modeling a graded proportion (e.g., defining an approximate analogy), other extensions should be considered for accommodating unknown values, or non-applicable attributes [68].

For those proportions that satisfy the equation solving property, they may be used to complete an existing pattern, taking into account some regularities with respect to an existing set of data, then paving the way to a machine learning technique, similar in nature to the well known k-nearest neighbor technique, but offering many more options and better results. From a practical viewpoint, it has been shown for Boolean features in $[3,50]$, that this equation solving process, using only the analogical proportion, can be the basis of a new classification technique. In that case, the missing information for the 4th item $d$ is only its class: $d$ is then classified according to analogical patterns extracted from the data at hand by solving the corresponding analogical equation. This technique has been successfully extended to numerical features [70], thanks to a multi-valued logic extension of $A$ and $P$. Moreover, a similar approach has been shown to be able to solve Raven Progressive Matrices tests [13]. 


\section{References}

[1] N. Barbot and L. Miclet. La proportion analogique dans les groupes: applications aux permutations et aux matrices. Technical Report 1914, IRISA, July 2009.

[2] M. Bayoudh, H. Prade, and G. Richard. A Kolmogorov complexity view of analogy: from logical modeling to experimentations. In M. Bramer, M. Petridis, and A. Hopgood, editors, Proc. 29th SGAI Int. Conf. on Innovative Techniques and Applications of Artificial Intelligence, Cambridge, 14-16 Dec. 2010, pages 93-106. Springer, 2011.

[3] S. Bayoudh, L. Miclet, and A. Delhay. Learning by analogy: A classification rule for binary and nominal data. Proc. 20th Int. Conf. on Artificial Intelligence (IJCAI'07), pages 678-683, 2007.

[4] S. Bayoudh, L. Miclet, A. Delhay, and H. Mouchère. De l'utilisation de la proportion analogique en apprentissage artificiel. In Actes des Journées Inteligence Artificielle Fondamentale (IAF'07), 2007.

[5] J.-Y. Béziau. New light on the square of oppositions and its nameless corner. Logical Investigations, 10:218-233, 2003.

[6] J.-Y. Béziau. The power of the hexagon. Logica Universalis, 6 (1-2):1-43, 2012.

[7] R. Blanché. Sur l'opposition des concepts. Theoria, 19:89-130, 1953.

[8] R. Blanché. Structures Intellectuelles. Essai sur l'Organisation Systématique des Concepts. Vrin, Paris, 1966.

[9] D. Bollegala, T. Goto, N. Tuan Duc, and M. Ishizuka. Improving relational similarity measurement using symmetries in proportional word analogies. Information Processing and Management, to appear in 2012.

[10] F. Cajori. William Oughtred: A Great Seventeenth-Century Teacher of Mathematics. Open Court Publishing Company, Chicago and London, 1916.

[11] N. Chater. The search for simplicity: A fundamental cognitive principle? In The Quarterly J. Experimental Psychology, volume 52 (A), pages 273-302, 1999.

[12] A. Cornuéjols. Analogy as minimization of description length. In G. Nakhaeizadeh and C. C. Taylor, editors, Machine Learning and Statistics: The Interface. Wiley and Sons, 1996.

[13] W. Correa, H. Prade, and G. Richard. When intelligence is just a matter of copying. In L. De Raedt et al., editor, Proc. 20th Europ. Conf. on Artificial Intelligence, Montpellier, Aug. 27-31, pages 276-281. IOS Press, 2012.

[14] M. Dastani, B. Indurkhya, and R. Scha. Analogical projection in pattern perception. Journal of Experimental and Theoretical Artificial Intelligence, 15(4):489 - 511, 2003.

[15] T. R. Davies and Russell S. J. A logical approach to reasoning by analogy. In J. P. McDermott, editor, Proc. 10th Int. Joint Conf. on Artificial Intelligence (IJCAI'87), Milan, pages 264-270. Morgan Kaufmann, 1987.

[16] B. De Finetti. La logique des probabilités. In Congrès International de Philosophie Scientifique, pages 1-9, Paris, France, 1936. Hermann et Cie.

[17] M. Dorolle. Le raisonnement par analogie. PUF, Paris, 1949.

[18] F. Douay-Soublin. La contre-analogie. Réflexion sur la récusation de certaines analogies pourtant bien formées cognitivement. Texto !, 1999.

[19] D. Dubois and H. Prade. Conditional objects as nonmonotonic consequence relationships. IEEE Trans. on Systems, Man and Cybernetics, 24:1724-1740, 1994. 
[20] D. Dubois and H. Prade. From Blanché's hexagonal organization of concepts to formal concept analysis and possibility theory. Logica Universalis, 6 (1-2):149-169, 2012 .

[21] P. Durrenberger and J. W. Morrison. A theory of analogy. J. of Anthropological Research, 33(4):372-387, 1977.

[22] T. G. Evans. A heuristic program to solve geometry-analogy problems. In Proc. A.F.I.P. Spring Joint Computer Conf., volume 25, pages 5-16, 1964.

[23] B. Falkenhainer, K. D. Forbus, and D. Gentner. The structure-mapping engine: algorithm and examples. Artif. Intell., 41(1):1-63, 1989.

[24] K. Forbus, A. Lovett, K. Lockwood, J. Wetzel, C. Matuk, B. Jee, and J. Usher. Cogsketch. In AAAI'08: Proc. 23rd national Conf. on Artificial intelligence, pages 1878-1879. AAAI Press, 2008.

[25] R. M. French. The computational modeling of analogy-making. Trends in Cognitive Sciences, 6(5):200 - 205, 2002.

[26] D. Gentner. Structure-mapping: A theoretical framework for analogy. Cognitive Science, 7(2):155-170, 1983.

[27] D. Gentner, K. J. Holyoak, and B. N. Kokinov. The Analogical Mind: Perspectives from Cognitive Science. Cognitive Science, and Philosophy. MIT Press, Cambridge, MA, 2001.

[28] N. L. Gick and K. J. Holyoak. Analogical problem solving. In Cognitive Psychology, volume 12, pages 306-335. July 1980.

[29] M. Guhe, A. Pease, A. Smaill, M. Martinez, M. Schmidt, H. Gust, K. Kühnberger, and U. Krumnack. A computational account of conceptual blending in basic mathematics. Cognitive Systems Research (Special Issue on Complex Cognition), 12(34):249-265, September-December 2011.

[30] H. Gust, K. Kühnberger, and U. Schmid. Metaphors and heuristic-driven theory projection (hdtp). Theoretical Computer Science, 354(1):98 - 117, 2006.

[31] D. H. Helman, editor. Analogical Reasoning: Perspectives of Artificial Intelligence. Cognitive Science, and Philosophy. Kluwer, Dordrecht, 1988.

[32] M. Hesse. On defining analogy. Proceedings of the Aristotelian Society, 60:79-100, 1959.

[33] D. Hofstadter and M. Mitchell. The Copycat project: A model of mental fluidity and analogy-making. In D. Hofstadter and The Fluid Analogies Research Group, editors, Fluid Concepts and Creative Analogies: Computer Models of the Fundamental Mechanisms of Thought, pages 205-267, New York, NY, 1995. Basic Books, Inc.

[34] K. J. Holyoak and P. Thagard. Analogical mapping by constraint satisfaction. Cognitive Science, 13:295-355, 1989.

[35] W. James. The Principles of Psychology. New York: Holt, 1890.

[36] S. Klein. Whorf transforms and a computer model for propositional/appositional reasoning. In Proc. of the Applied Mathematics colloquium, Univ. of Bielefeld, 1977.

[37] S. Klein. Culture, mysticism \& social structure and the calculation of behavior. In Proc. 5th Europ. Conf. in Artificial Intelligence (ECAI'82), Paris, pages 141-146, 1982. 
[38] S. Klein. Analogy and mysticism and the structure of culture (and Com-ments \& Reply). Current Anthropology, 24 (2):151-180, 1983.

[39] S. Klein. The analogical foundations of creativity in language, culture \& the arts: the upper paleolithic to 2100ce. In P. McKevitt, C. Mulvihill, and S. O' Nuallin, editors, Language, Vision 83 Music, pages 347-371. Amsterdam: John Benjamin, 2002.

[40] R. E. Kling. A paradigm for reasoning by analogy. In Proc. 2nd Int. Joint Conf. on Artificial Intelligence (IJCAI'71), Imperial College, London, Sept. 1-3, pages 568585, 1971.

[41] M. Kunda, K. McGreggor, and A. Goel. A computational model for solving problems from the ravens progressive matrices intelligence test using iconic visual representations. Cognitive Systems Research, pages 47-66, 2013.

[42] P. Langlais, F. Yvon, and P. Zweigenbaum. Analogical translation of medical words in different languages. In GoTAL, pages 284-295, 2008.

[43] Y. Lepage. Analogy and formal languages. Electr. Notes Theor. Comput. Sci., 53, 2001.

[44] Y. Lepage. De l'analogie rendant compte de la commutation en linguistique. 2003. Habilitation thesis, May 23, CLIPS / IMAG, Univ. Joseph-Fourier - Grenoble I.

[45] Y. Lepage and S. Ando. Saussurian analogy: a theoretical account and its application. In Proc. 16th Int. Conf. on Computational Linguistics (COLING'96), Copenhagen, Aug. 5-9, 1996, pages 717-722, 1996.

[46] Y. Lepage, J. Migeot, and E. Guillerm. A measure of the number of true analogies between chunks in japanese. In Z. Vetulani and H. Uszkoreit, editors, Human Language Technology. Challenges of the Information Society, 3rd Language and Technology Conf., LTC 2007, Poznan, Poland, October 5-7, 2007, Revised Selected Papers, volume 5603 of LNCS, pages 154-164. Springer, 2009.

[47] F. Lorrain. Réseaux Sociaux et Classifications Sociales. Essai sur l'Algèbre et la Géométrie des Structures Sociales. Hermann, Paris, 1975.

[48] A. Lovett, K. Forbus, and J. Usher. A structure-mapping model of Raven's progressive matrices. In Proc. of the 32nd Annual Conf. of the Cognitive Science Society, Portland, OR, 2010.

[49] E. Melis and M. Veloso. Analogy in problem solving. In Handbook of Practical Reasoning: Computational and Theoretical Aspects. Oxford Univ. Press, 1998.

[50] L. Miclet, S. Bayoudh, and A. Delhay. Analogical dissimilarity: definition, algorithms and two experiments in machine learning. JAIR, 32, pages 793-824, 2008.

[51] L. Miclet and A. Delhay. Relation d'analogie et distance sur un alphabet défini par des traits. Technical Report 1632, IRISA, Rennes, July 2004.

[52] L. Miclet and H. Prade. Handling analogical proportions in classical logic and fuzzy logics settings. In Proc. 10th Eur. Conf. on Symbolic and Quantitative Approaches to Reasoning with Uncertainty (ECSQARU'09), Verona, pages 638-650. Springer, LNCS 5590, 2009.

[53] A. Moretti. Why the logical hexagon? Logica Universalis, 6 (1-2):69-107, 2012.

[54] D. P. O'Donoghue, A. J. Bohan, and M. T. Keane. Seeing things: Inventive reasoning with geometric analogies and topographic maps. New Generation Comput., 24(3):267-288, 2006. 
[55] J. Piaget. Classe, Relations et Nombres. Essai sur les Groupements de la Logistique et sur la Réversibilité de la Pensée. Vrin, Paris, 1942.

[56] J. Piaget. Traité de Logique : Essai de Logistique Opératoire. Dunod, 1949.

[57] J. Piaget. Essai sur les transformations des opérations logiques: les 256 opérations ternaires de la logique bivalente des propositions. Presses Univ. de France, Paris, 1952.

[58] J. Piaget. Logic and Psychology. Manchester Univ. Press, 1953.

[59] G. D. Plotkin. A Note on Inductive Generalization. Machine Intelligence, 5:153-163, 1970.

[60] H. Prade and G. Richard. Analogy, paralogy and reverse analogy: Postulates and inferences. In Proc. 32nd Ann. Conf. on Artif. Intellig. (KI 2009), Paderborn, volume LNAI 5803, pages 306-314. Springer, 2009.

[61] H. Prade and G. Richard. Analogical proportions: another logical view. In M. Bramer, R. Ellis, and M. Petridis, editors, Research and Development in Intelligent Systems XXVI, Proc. 29th Ann. Int. Conf. on AI (SGAI'09), Cambridge, UK, December 2009, pages 121-134. Springer, 2010.

[62] H. Prade and G. Richard. Logical proportions - typology and roadmap. In E. Hüllermeier, R. Kruse, and F. Hoffmann, editors, Computational Intelligence for Knowledge-Based Systems Design: Proc. 13th Int. Conf. on Information Processing and Management of Uncertainty (IPMU'10), Dortmund, June 28 - July 2, volume 6178 of $L N C S$, pages 757-767. Springer, 2010.

[63] H. Prade and G. Richard. Multiple-valued logic interpretations of analogical, reverse analogical, and paralogical proportions. In Proc. 40th IEEE Int. Symp. on MultipleValued Logic (ISMVL'10), Barcelona, May 26-28, pages 258-263, 2010.

[64] H. Prade and G. Richard. Reasoning with logical proportions. In Proc. 12th Int. Conf. on Principles of Knowledge Representation and Reasoning, KR 2010, Toronto, Ontario, Canada, May 9-13, 2010 (F. Z. Lin, U. Sattler, M. Truszczynski, eds.), pages 545-555. AAAI Press, 2010.

[65] H. Prade and G. Richard. Homogeneous logical proportions: Their uniqueness and their role in similarity-based prediction. In G. Brewka, T. Eiter, and S. A. McIlraith, editors, Proc. 13th Int. Conf. on Principles of Knowledge Representation and Reasoning (KR12), Roma, June 10-14, pages 402-412. AAAI Press, 2012.

[66] H. Prade and G. Richard. Logical proportions - further investigations. In S. et al. Greco, editor, Computational Intelligence for Knowledge-Based Systems Design, Proc. 14th Int. Conf. on Inform. Process. and Management of Uncertainty in Knowledge-Based Systems (IPMU'12), Catania, Italy, July 9-13, volume 297 of CCIS, pages 208-218. Springer, 2012.

[67] H. Prade and G. Richard. On the hexagonal organization of concepts related to the ideas of similarity and analogy. In J.-Y. Béziau and K. Gan-Krzywoszyńska, editors, Handbook of abstracts of the 3rd World Congress on the Square of Opposition, pages 75, Beirut, June 26-30, 2012.

[68] H. Prade and G. Richard. Analogical proportions and multiple-valued logics. In L. C. van der Gaag, editor, Proc. 12th Eur. Conf. on Symb. and Quantit. Appr. to Reasoning with Uncertainty (ECSQARU'13), Utrecht, Jul. 7-10, LNAI 7958, pages 497-509. Springer, 2013. 
[69] H. Prade and G. Richard. Picking the one that does not fit - A matter of logical proportions. In Proc. 8th Conf. Europ. Soc. for Fuzzy Logic and Technology (EUSFLAT), Milano, Sept. 11-13, 2013.

[70] H. Prade, G. Richard, and B. Yao. Enforcing regularity by means of analogy-related proportions-a new approach to classification. Int. J. of Computer Information Systems and Industrial Management Applications, 4:648-658, 2012.

[71] T. Sakaguchi, Y. Akaho, K. Okada, T. Date, T. Takagi, N. Kamimaeda, M. Miyahara, and T. Tsunoda. Recommendation system with multi-dimensional and parallel-case four-term analogy. Proc. IEEE Int. Conf. on Systems, Man, and Cybernetics (SMC), Anchorage, Oct. 9-12, pages 3137 - 3143, 2011.

[72] U. Schmid, H. Gust, K. Kühnberger, and J. Burghardt. An algebraic framework for solving proportional and predictive analogies. Eur. Conf. Cogn. Sci. 295-300, 2003.

[73] D. E. Smith. History of Mathematics, Vol. 1, reprinted by. Dover, New York, 1958.

[74] J. F. Sowa and A. K. Majumdar. Analogical reasoning. In Proc. Int. Conf. on Conceptual Structures, pages 16-36. Springer, LNAI 2746, 2003.

[75] N. Stroppa and F. Yvon. An analogical learner for morphological analysis. In Online Proc. 9th Conf. Comput. Natural Language Learning (CoNLL-2005), pages 120-127, 2005.

[76] N. Stroppa and F. Yvon. Analogical learning and formal proportions: Definitions and methodological issues. ENST, Paris, Tech. Rep. TR-D004, 2005.

[77] N. Stroppa and F. Yvon. Du quatrième de proportion comme principe inductif : une proposition et son application à l'apprentissage de la morphologie. Traitement Automatique des Langues, 47(2):1-27, 2006.

[78] P. D. Turney. A uniform approach to analogies, synonyms, antonyms, and associations. In COLING, pages 905-912, 2008.

[79] P. D. Turney and M. L. Littman. Corpus-based learning of analogies and semantic relations. Mach. Learn., 60(1-3):251-278, 2005.

[80] A. Tversky. Features of similarity. In Psychological Review, number 84, pages 327352. American Psychological Association, 1977.

[81] T. Veale and M. Keane. The competence of sub-optimal theories of structure mapping on hard analogies. Proc. Int. Joint C. on Artificial Intelligence (IJCAI'97), 15(1):232-237, 1997.

[82] P. H. Winston. Learning and reasoning by analogy. Commun. ACM, 23(12):689-703, 1980. 


\section{ANNEX A: Truth tables}

We do not repeat here the truth tables of homogeneous proportions which have been largely investigated through this paper. Moreover, in order to reduce the size of the tables, we omit the $\wedge$ symbol and we separate the 2 equalities defining a proportion by a simple vertical bar $\mid$.

- We start with Table 9 showing the 16 conditional proportions.

- Then we have 20 hybrids proportions given in Table 10.

- We have 32 semi-hybrids given in Table 11.

- We end the serie with Table 12 showing degenerated proportions, i.e., the proportions where 2 indicators among 4 are identical. They are easy to build up and we only show 3 of them. All the remaining ones are easily deducible.

- Table 13 shows the 15 proportions satisfying the full identity property $(\wedge$ are omitted). A, R, P are on the first line, while degenerated ones are the last four.

- We exhibit the 4 conditional and the 4 hybrid proportions satisfying the symmetry property in Table 14 . When adding the homogeneous proportions $A, R, P, I$, we get a total number of 12 proportions.

\begin{tabular}{|c|c|c|c|}
\hline formula & notation & formula & notation \\
\hline$a b \equiv c d \mid a \bar{b} \equiv c \bar{d}$ & $b|a:: d| c$ & $\bar{a} \bar{b} \equiv c d \mid a \bar{b} \equiv c \bar{d}$ & $a|\bar{b}:: d| c$ \\
\hline$a b \equiv c d \mid a \bar{b} \equiv \bar{c} d$ & $b|a:: c| d$ & $\bar{a} \bar{b} \equiv c d \mid a \bar{b} \equiv \bar{c} d$ & $a|\bar{b}:: c| d$ \\
\hline$a b \equiv c d \mid \bar{a} b \equiv c \bar{d}$ & $a|b:: d| c$ & $\bar{a} \bar{b} \equiv c d \mid \bar{a} b \equiv c \bar{d}$ & $b|\bar{a}:: d| c$ \\
\hline$a b \equiv c d \mid \bar{a} b \equiv \bar{c} d$ & $a|b:: c| d$ & $\bar{a} \bar{b} \equiv c d \mid \bar{a} b \equiv \bar{c} d$ & $b|\bar{a}:: c| d$ \\
\hline$a b \equiv \bar{c} \bar{d} \mid a \bar{b} \equiv c \bar{d}$ & $b|a:: c| \bar{d}$ & $\bar{a} \bar{b} \equiv \bar{c} \bar{d} \mid a \bar{b} \equiv c \bar{d}$ & $a|\bar{b}:: c| \bar{d}$ \\
\hline$a b \equiv \bar{c} \bar{d} \mid a \bar{b} \equiv \bar{c} d$ & $b|a:: d| \bar{c}$ & $\bar{a} \bar{b} \equiv \bar{c} \bar{d} \mid a \bar{b} \equiv \bar{c} d$ & $a|\bar{b}:: d| \bar{c}$ \\
\hline$a b \equiv \bar{c} \bar{d} \mid \bar{a} b \equiv c \bar{d}$ & $a|b:: c| \bar{d}$ & $\bar{a} \bar{b} \equiv \bar{c} \bar{d} \mid \bar{a} b \equiv c \bar{d}$ & $b|\bar{a}:: c| \bar{d}$ \\
\hline$a b \equiv \bar{c} \bar{d} \mid \bar{a} b \equiv \bar{c} d$ & $a|b:: d| \bar{c}$ & $\bar{a} \bar{b} \equiv \bar{c} \bar{d} \mid \bar{a} b \equiv \bar{c} d$ & $b|\bar{a}:: d| \bar{c}$ \\
\hline
\end{tabular}

TABLE 9. 16 conditional proportions

\begin{tabular}{|c|c|c|c|c|}
\hline$a b \equiv c \bar{d}$ & $\bar{a} \bar{b} \equiv \bar{c} d$ & $\bar{a} b \equiv c d \mid a \bar{b} \equiv \bar{c} \bar{d}$ & $a b \equiv \bar{c} d \mid \bar{a} \bar{b} \equiv c \bar{d}$ & $\bar{a} b \equiv \bar{c} \bar{d} \mid a \bar{b} \equiv c d$ \\
\hline & & $a \bar{b}$ & $a b \equiv \bar{c} d \mid \bar{a} b \equiv \bar{c} \bar{d}$ & $a b \equiv \bar{c} d \mid a \bar{b} \equiv \bar{c} \bar{d}$ \\
\hline & & $\bar{a} \bar{b}$ & $\bar{a} \bar{b} \equiv \bar{c} d$ & $\bar{a} \bar{b} \equiv \bar{c} d \mid a \bar{b}$ \\
\hline & & $a b \equiv$ & $\bar{a} b \equiv \bar{c} \bar{d}$ & $a b \equiv c \bar{a}$ \\
\hline$\equiv$ & $\bar{a} b$ & $\bar{a} \bar{b} \equiv c \bar{d} \mid a \bar{b} \equiv c d$ & $\bar{a} \bar{b} \equiv c \bar{d} \mid \bar{a} b \equiv \bar{c} \bar{d}$ & $\bar{a} \bar{b} \equiv c \bar{d} \mid a \bar{b} \equiv$ \\
\hline
\end{tabular}

TABLE 10. 20 hybrid proportions 


\begin{tabular}{c||c}
$S \equiv S^{\prime} \mid S \equiv D$ & $S \equiv S^{\prime} \mid S \equiv D$ \\
$a b \equiv c d \mid \bar{a} \bar{b} \equiv \bar{c} d$ & $a b \equiv c d \mid \bar{a} \bar{b} \equiv c \bar{d}$ \\
$a b \equiv \bar{c}|\bar{a}| \bar{b} \equiv \bar{c} d$ & $a b \equiv \bar{c} \bar{d} \mid \bar{a} \bar{b} \equiv c \bar{d}$ \\
$\bar{a} \bar{b} \equiv c d \mid a b \equiv \bar{c} d$ & $\bar{a} \bar{b} \equiv c d \mid \bar{a} \bar{b} \equiv c \bar{d}$ \\
$\bar{a} \bar{b} \equiv \bar{c} \bar{d} \mid a b \equiv \bar{c} d$ & $\bar{a} \bar{b} \equiv \bar{c} \bar{d} \mid \bar{a} \bar{b} \equiv c \bar{d}$ \\
\hline$D \equiv D^{\prime} \mid S \equiv D$ & $D \equiv D^{\prime} \mid S \equiv D$ \\
$a \bar{b} \equiv c \bar{d} \mid \bar{a} \bar{b} \equiv \bar{c} d$ & $a \bar{b} \equiv \bar{c} d \mid \bar{a} \bar{b} \equiv c \bar{d}$ \\
$a \bar{b} \equiv c \bar{d} \mid \bar{a} \bar{b} \equiv \bar{c} d$ & $a \bar{b} \equiv \bar{c} d \mid \bar{a} \bar{b} \equiv c \bar{d}$ \\
$\bar{a} b \equiv c \bar{d} \mid a b \equiv \bar{c} d$ & $\bar{a} b \equiv \bar{c} d \mid a b \equiv c \bar{d}$ \\
$\bar{a} b \equiv c \bar{d} \mid a b \equiv \bar{c} d$ & $\bar{a} b \equiv \bar{c} d \mid a b \equiv c \bar{d}$
\end{tabular}

\begin{tabular}{c||c}
$S \equiv S^{\prime} \mid D \equiv S$ & $S \equiv S^{\prime} \mid D \equiv S$ \\
$a b \equiv c d \mid a \bar{b} \equiv \bar{c} \bar{d}$ & $a b \equiv c d \mid \bar{a} b \equiv \bar{c} \bar{d}$ \\
$a b \equiv \bar{c} \bar{d} \mid a \bar{b} \equiv c d$ & $a b \equiv \bar{c} \bar{d} \mid \bar{a} b \equiv c d$ \\
$\bar{a} \bar{b} \equiv c d \mid a \bar{b} \equiv \bar{c} \bar{d}$ & $\bar{a} \bar{b} \equiv c d \mid \bar{a} b \equiv \bar{c} \bar{d}$ \\
$\bar{a} \bar{b} \equiv \bar{c} \bar{d} \mid a \bar{b} \equiv c d$ & $\bar{a} \bar{b} \equiv \bar{c} \bar{d} \mid \bar{a} b \equiv c d$ \\
\hline$D \equiv D^{\prime} \mid D \equiv S$ & $D \equiv D^{\prime} \mid D \equiv S$ \\
$a \bar{b} \equiv c \bar{d} \mid \bar{a} b \equiv c d$ & $a \bar{b} \equiv \bar{c} d \mid \bar{a} b \equiv c d$ \\
$a \bar{b} \equiv c \bar{d} \mid \bar{a} b \equiv \bar{c} \bar{d}$ & $a \bar{b} \equiv \bar{c} d \mid \bar{a} b \equiv \bar{c} \bar{d}$ \\
$\bar{a} b \equiv c \bar{d} \mid a \bar{b} \equiv c d$ & $\bar{a} b \equiv \bar{c} d \mid a \bar{b} \equiv c d$ \\
$\bar{a} b \equiv c \bar{d} \mid a \bar{b} \equiv \bar{c} \bar{d}$ & $\bar{a} b \equiv \bar{c} d \mid a \bar{b} \equiv \bar{c} \bar{d}$
\end{tabular}

TABLE 11. 32 semi-hybrid proportions

$$
a b \equiv c d|a b \equiv \bar{c} d\|a b \equiv c d|a b \equiv c \bar{d} \| a b \equiv c d| a b \equiv \bar{c} \bar{d}
$$

TABle 12. Degenerated proportions (a sample among 48)

$$
\begin{array}{l|l||l||l|l|l}
\bar{a} b \equiv \bar{c} d & a \bar{b} \equiv c \bar{d} & \bar{a} b \equiv c \bar{d} & a \bar{b} \equiv \bar{c} d & a b \equiv c d & \bar{a} \bar{b} \equiv \bar{c} \bar{d} \\
\bar{a} \bar{b} \equiv \bar{c} \bar{d} & a \bar{b} \equiv c \bar{d} & \bar{a} \bar{b} \equiv \bar{c} \bar{d} & a \bar{b} \equiv \bar{c} d & a \bar{b} \equiv c \bar{d} & a b \equiv c d \\
\bar{a} \bar{b} \equiv \bar{c} \bar{d} & \bar{a} b \equiv \bar{c} d & \bar{a} b \equiv c \bar{d} & a b \equiv c d & \bar{a} b \equiv \bar{c} d & a b \equiv c d \\
\bar{a} \bar{b} \equiv \bar{c} \bar{d} & \bar{a} b \equiv c \bar{d} & a \bar{b} \equiv \bar{c} d & a b \equiv c d & a \bar{b} \equiv \bar{c} d & \bar{a} b \equiv \bar{c} d \\
a \bar{b} \equiv \bar{c} d & a \bar{b} \equiv c \bar{d} & \bar{a} b \equiv c \bar{d} & a \bar{b} \equiv c \bar{d} & \bar{a} b \equiv \bar{c} d & \bar{a} b \equiv c \bar{d}
\end{array}
$$

TABLE 13. The 15 proportions satisfying full identity property

$$
\begin{aligned}
& a b \equiv c d|\bar{a} b \equiv \bar{c} d\|a b \equiv c d|a \bar{b} \equiv c \bar{d}\|\bar{a} \bar{b} \equiv \bar{c} \bar{d}|\bar{a} b \equiv \bar{c} d \| \bar{a} \bar{b} \equiv \bar{c} \bar{d}| a \bar{b} \equiv c \bar{d} \\
& a \bar{b} \equiv c d|a b \equiv c \bar{d}\|\bar{a} b \equiv c d|a b \equiv \bar{c} d\|\bar{a} \bar{b} \equiv \bar{c} d|\bar{a} b \equiv \bar{c} \bar{d} \| \bar{a} \bar{b} \equiv c \bar{d}| a \bar{b} \equiv \bar{c} \bar{d}
\end{aligned}
$$

TABLE 14. The 4 conditional (1st line) and 4 hybrid proportions satisfying symmetry

\section{ANNEX B: Proofs}

The proofs are mainly obtained by a rigorous examination of the way the proportions are built up. Since they are made up with a pair of equivalences between indicators, let us investigate first these diverse equivalences. We have the following property:

Lemma 1 An equivalence between indicators has exactly 10 valid valuations.

Proof: Such an equivalence $e q \triangleq I_{a, b} \equiv I_{c, d}$ is satisfied only when it matches one of the 2 patterns $1=1$ or $0=0$ : due to the fact that 0 is an absorbing value for $\wedge$, these patterns correspond to the 10 values shown in Table 15 for the literals involved in the indicators (with obvious notation). Any other valuation does not 
match anyone of the 2 previous patterns and will lead to the truth value 0 for the equivalence $e q$.

$\begin{array}{ccccc}\text { literal 1 } & \text { literal 2 } & \text { literal 3 } & \text { literal 4 } & \text { pattern } \\ 1 & 1 & 1 & 1 & 1=1 \\ 0 & 1 & 0 & 1 & 0=0 \\ 0 & 1 & 1 & 0 & 0=0 \\ 0 & 1 & 0 & 0 & 0=0 \\ 1 & 0 & 0 & 1 & 0=0 \\ 1 & 0 & 1 & 0 & 0=0 \\ 1 & 0 & 0 & 0 & 0=0 \\ 0 & 0 & 0 & 1 & 0=0 \\ 0 & 0 & 1 & 0 & 0=0 \\ 0 & 0 & 0 & 0 & 0=0\end{array}$

TABLE 15. The 10 valid literal valuations for an equivalence between indicators

Lemma 2 Two equivalences between indicators have the same truth table iff they are identical.

Proof: It is sufficient to show that if 2 equalities $e q_{1}$ and $e q_{2}$ have the same truth table, then they are syntactically identical. In other terms, we have to prove that $e q_{1} \equiv e q_{2}$ implies $e q_{1}={ }_{I d} e q_{2}$. We can assume for instance without loss of generality that $e q_{1}$ contains $a$ but $e q_{2}$ contains $\bar{a}$. Let us consider the unique valuation $v$ such that $v\left(e q_{1}\right)=1$ with the pattern $1=1$. This valuation $v$ is such that $v(a)=1$. By hypothesis, $v\left(e q_{2}\right)=1$ but in that case with the pattern $0=0$ since $v(\bar{a})=0$. Let us now modify $v$ into $v^{\prime}$ such that $v^{\prime}(a)=0, v^{\prime}(c)=v(c), v^{\prime}(d)=v(d)$ and $v^{\prime}(b)=v(b)$. Obviously $v^{\prime}$ does not validate $e q_{1}$ but still validates $e q_{2}$ which contradicts the hypothesis. Then the expected result.

Lemma 3 When an equivalence involves a similarity indicator and a dissimilarity indicator, then it cannot be satisfied for both valuations 0000 and 1111.

Proof: First of all, we observe that any of the 2 valuations 0000 or 1111 will assign the value 0 to any dissimilarity indicator. On the opposite, one of the 2 previous valuations will assign the value 1 to any similarity indicator. Let us suppose we have an equivalence $S_{a, b} \equiv D_{c, d}$, the 2 previous remarks show that at least one of the 2 valuations will assign the value 0 to the equivalence.

Lemma 4 An equivalence involving only dissimilarity indicators is satisfied by both 1111 and 0000 . 
Proof: This comes from the fact that these 2 valuations allocate the value 0 to any dissimilarity indicator.

Now, we have a lemma showing that certain classes of valuation cannot be satisfied by an equivalence between indicators, and then certainly not by a logical proportion which is built up with 2 such equivalences.

Lemma 5 A logical proportion cannot satisfies the classes of valuation $\{0111,1011$, $1101,1110\}$ or the classe $\{1000,0100,0010,0001\}$.

Proof: It is enough to show that this is the case for an equivalence between indicators. So let us consider such an equivalence $l_{1} \wedge l_{2} \equiv l_{3} \wedge l_{4}$. If this equivalence is valid for $\{0111,1011\}$, it means that its truth value does not change when we switch the truth value of the 2 first literals from 0 to 1 : there are only 2 indicators for $a$ and $b$ satisfying this requirement: $a \wedge b$ and $\bar{a} \wedge \bar{b}$. On top of that, if this equivalence is still valid for $\{1101,1110\}$, it means that its truth value does not change when we switch the truth value of the 2 last literals from 0 to 1 : there are only 2 indicators for $c$ and $d$ satisfying this requirement: $c \wedge d$ and $\bar{c} \wedge \bar{d}$. Then the equivalence $l_{1} \wedge l_{2} \equiv l_{3} \wedge l_{4}$ is just $a \wedge b \equiv c \wedge d, a \wedge b \equiv \bar{c} \wedge \bar{d}, a \wedge b \equiv \bar{c} \wedge \bar{d}$ or $\bar{a} \wedge \bar{b} \equiv \bar{c} \wedge \bar{d}$. None of these equivalences satisfies the whole class $\{0111,1011,1101,1110\}$. The same reasoning applies for the other class.

Before starting with the proofs of the main properties, let us summarize in a final lemma some straightforward properties of the indicators:

Lemma 6 Given a pair $(a, b)$ with $S_{(a, b)}$ and $S_{(a, b)}^{\prime}$ (resp. $D_{(a, b)}$ and $\left.D_{(a, b)}^{\prime}\right)$ the 2 similarity (resp. dissimilarity) indicators, and a valuation $\sigma$ :

- $\sigma(a) \neq \sigma(b)$ iff $\sigma\left(S_{(a, b)}\right)=\sigma\left(S_{(a, b)}^{\prime}\right)=0$ (or equivalently if $\sigma\left(S_{(a, b)}\right)=1$ or $\sigma\left(S_{(a, b)}^{\prime}\right)=1$ then $\left.\sigma(a)=\sigma(b)\right)$

- $\sigma(a)=\sigma(b)$ iff $\sigma\left(D_{(a, b)}\right)=\sigma\left(D_{(a, b)}^{\prime}\right)=0$ (or equivalently if $\sigma\left(D_{(a, b)}\right)=1$ or $\sigma\left(D_{(a, b)}^{\prime}\right)=1$ then $\left.\sigma(a) \neq \sigma(b)\right)$

- $\sigma\left(S_{(a, b)}\right)$ and $\sigma\left(S_{(a, b)}^{\prime}\right)$ (resp. $\sigma\left(D_{(a, b)}\right)$ and $\sigma\left(D_{(a, b)}^{\prime}\right)$ ) cannot be equal to 1 simultaneously.

This lemma justifies once again the name similarity (resp. dissimilarity) for the respective indicators: for instance, we observe the identity of 2 objects $\sigma(a)$ and $\sigma(b)$ when at least one similarity indicator is equal to 1 (and the other one is equal to 0 ) or equivalently, when their 2 dissimilarity indicators are equal to 0 .

Proposition 1: The truth table of a logical proportion has 6 and only 6 lines with truth value 1.

Proof: Since a logical proportion $T$ is the conjunction $e q_{1} \wedge e q_{2}$ of 2 equalities between indicators, with $e q_{1} \neq e q_{2}$, it appears from Lemma 1 that $T$ has a maximum of 10 valid valuations and a minimum of 4 valid valuations. Let us start from eq1, having 10 valid valuations which are candidate to validate $T$. Obviously, adding 
$e q_{2}$ to $e q_{1}$ will reduce the number of valid valuations for $T$. Let us assume $e q_{2}$ differs from $e q_{1}$ with only one literal (or negation operator). This is then a degenerated proportion. Without loss of generality, we can consider that the difference between $e q_{1}$ and $e q_{2}$ occurs on the first literal meaning $e q_{1}$ is $a \wedge l_{2} \equiv l_{3} \wedge l_{4}$ and $e q_{2}$ is $\bar{a} \wedge l_{2} \equiv l_{3} \wedge l_{4}$ or vice versa. It is then quite clear that the first valuation 1111 valid for $e q_{1}$ is not valid any more for $T$. It remains 9 candidates valuations. Finally any valuation starting with 01 is not valid any more and we have 3 such valuations. All the 6 remaining valuations are still valid for $T$. Which ends the proof when the 2 equalities differ from one negation (i.e. one literal).

Now when they differ from 2 literals, two cases have to be considered:

- either the 2 literals where $e q_{1}$ differs from $e q_{2}$ are on the same side of an equivalence i.e. $e q_{2}$ is $l_{1}^{\prime} \wedge l_{2}^{\prime} \equiv l_{3} \wedge l_{4}$ (degenerated proportion)

- or they are on different side i.e. $e q_{2}$ is $l_{1}^{\prime} \wedge l_{2} \equiv l_{3}^{\prime} \wedge l_{4}$.

In the first case, the valuations 1111,0010, 0001 and 0000 are not valid any more, but all other ones remain valid. In the second case, the valuations 0100, 0110, 1001 and 0001 are not valid anymore, but all the other ones remain valid. We are done for the case of 2 differences. When they differ from 3 literals, let us suppose $l_{4}$ appears in both equivalence, the valuations 1001,0101,0010 and 0000 are not valid anymore and we stick with the 6 remaining ones. In the case where all the literals are different, obviously the 4 valuations containing only one occurence of 1 are not valid anymore because they lead to an invalid pattern $0=1$ or $1=0$ for $e q_{2}$. And we have exactly 4 such valuations. It remains 6 valid valuations. Which ends the proof.

Proposition 2: The truth tables of the 120 proportions are all distinct.

Proof: We shall show that, when 2 proportions $T \triangleq e q_{1} \wedge e q_{2}$ and $T^{\prime} \triangleq e q_{1}^{\prime} \wedge e q_{2}^{\prime}$ have the same truth table, they are syntactically identical (up to a permutation of the 2 equalities). In other words $T \equiv T^{\prime}$ implies $T=_{I d} T^{\prime}$. Starting from $T \equiv T^{\prime}$ (i.e. $T$ and $T^{\prime}$ coincide on any valuation $\sigma$ ), if $e q_{1}$ is syntactically different from $e q_{1}^{\prime}$, we show that $e q_{1}$ is syntactically equal to $e q_{2}$. This will complete the proof as a similar reasoning will show that $e q_{2}$ is, in the same context, syntactically equal to $e q_{1}^{\prime}$.

In fact, if $e q_{1}$ is syntactically different from $e q_{1}^{\prime}$, we can assume for instance without loss of generality that $e q_{1}$ contains $a$ but $e q_{1}^{\prime}$ contains $\bar{a}$. Let us consider the unique valuation $\sigma$, validating $T$ and $T^{\prime}$, such that $\sigma\left(e q_{1}\right)=1$ with the pattern $1=1$. Necessarily, this valuation $\sigma$ is such that $\sigma(a)=1$. By hypothesis, $\sigma\left(e q_{1}^{\prime}\right)=1$ but in that case with the pattern $0=0$ since $\sigma(\bar{a})=0$. Let us now consider the new valuation $\sigma^{\prime}$ such that $\sigma^{\prime}(a)=0, \sigma^{\prime}(c)=\sigma(c), \sigma^{\prime}(d)=\sigma(d)$ and $\sigma^{\prime}(b)=\sigma(b)$. Obviously $\sigma^{\prime}(T)=\sigma^{\prime}\left(e q_{1}\right)=0$ but still $\sigma^{\prime}\left(e q_{1}^{\prime}\right)=1$ following the pattern $0=0$. The only option for having $\sigma^{\prime}(T)=\sigma^{\prime}\left(T^{\prime}\right)=0$ is thus to have $\sigma^{\prime}\left(e q_{2}^{\prime}\right)=0$ which means $a$ belongs to $e q_{2}^{\prime}$. Continuing the same reasoning, we show that $e q_{1}=_{I d} e q_{2}^{\prime}$ 
and we conclude that if $e q_{1} \neq e q_{1}^{\prime}$, necessarily $e q_{1}={ }_{I d} e q_{2}$. Which is the expected result.

Proposition 3: There are only 15 proportions satisfying full identity: 3 of them are homogeneous (they are $A, R$, and $P$ ), 8 of them are conditional proportions and the 4 remaining ones are degenerated.

Proof: Lemma 3 shows that none of the hybrid or semi-hybrid proportions can satisfy full identity since by definition such a proportion includes an equivalence between a similarity indicator and a dissimilarity indicator. Concerning the conditional ones, it is clear that the equivalence involving only dissimilarity indicators will be satisfied whatever the valuations (lemma 4 ), but the remaining equivalence involving similarity indicators will be satisfied iff it is of the form $a \wedge b \equiv c \wedge d$ or $\bar{a} \wedge \bar{b} \equiv \bar{c} \wedge \bar{d}$ which leads to exactly 8 conditional proportions. Finally, the same reasoning applies to degenerated proportions: only remain the proportions which do not involve an equivalence between a similarity indicator and a dissimilarity indicator. But now, if we remember that an indicator appears twice in a given degenerated proportion, the proportions involving only similarity indicators cannot lead to 1 for the 2 previous valuations since such a valuation can satisfy only one equivalence. It remains only the degenerated proportions involving only equalities between dissimilarity indicators. And we have exactly 4 such proportions: we fix for instance the first dissimilarity indicator (2 possibilities) as the left hand side of the 2 equalities and we build only one proportion with the right hand side indicators. We repeat the process but fixing now the right hand side of the equalities. And we are done with the 4 degenerated proportions. An immediate check allows to conclude for homogeneous proportions. This achieves the whole proof.

Proposition 4: There are 30 proportions validated with 1111 but not validated with 0000. Dually, there are also 30 proportions validated with 0000 but not with 1111. Proof: Due to the complete symmetry in the notation, it is sufficient to show the first part of the proposition. Lemma 4 tells us that any equivalence involving 2 dissimilarity indicators $D_{(a, b)} \equiv D_{(c, d)}$ is validated with both 1111 and 0000. On the opposite, an equivalence involving only similarity indicator $S_{(a, b)} \equiv S_{(c, d)}$ is validated by both of them $(a \wedge b \equiv c \wedge c$ and $\bar{a} \wedge \bar{b} \equiv \bar{c} \wedge \bar{d})$ or by none of them $(\bar{a} \wedge \bar{b} \equiv$ $c \wedge d$ and $a \wedge b \equiv \bar{c} \wedge \bar{d}$ ). This excludes the 4 homogeneous and the 16 conditional proportions as suitable candidates. Concerning the hybrid proportions, they obey 3 possible distinct patterns characterized by equivalences between similarity and dissimilarity indicators in their definitions. They are of the form:

$$
S_{(a, b)} \equiv D_{(c, d)} \wedge S_{(a, b)}^{\prime} \equiv D_{(c, d)}^{\prime}
$$

or

$$
D_{(a, b)} \equiv S_{(c, d)} \wedge D_{(a, b)}^{\prime} \equiv S_{(c, d)}^{\prime}
$$

or

$$
S_{(a, b)} \equiv D_{(c, d)} \wedge D_{(a, b)} \equiv S_{(c, d)}
$$


The first pattern, involving a similarity indicator on the left hand side of the 2 equalities, cannot lead to suitable proportions since the right hand side is a dissimilarity indicator having value 0 for the 2 valuations: this implies that at least one of the 2 equalities will not be satisfied whatever the valuation. The same reasoning applies with the second pattern where the similarity indicators appear on the right hand side of the equalities. It remains only the hybrid proportions where a similarity indicator appears as the left hand side of the first equivalence and as the right hand side of the second equivalence or vice-versa. If we consider only the valuation 1111 , the only suitable similarity indicators are $\bar{a} \wedge \bar{b}$ and $\bar{c} \wedge \bar{d}$ : and we have 4 choices for the remaining dissimilarity indicators leading to exactly 4 suitable hybrid proportions. A similar reasoning leads to 12 semi-hybrids and 14 degenerated (degenerated proportions are simpler to investigate since they make use of only 3 distinct indicators).

\section{Proposition 5:}

- There is no proportion simultaneously satisfying the 3 facts $T(a, a, b, b)$, $T(a, b, a, b)$ and $T(a, b, b, a)$.

- $A$ is the only proportion to satisfy $T(a, a, b, b)$ and $T(a, b, a, b)$.

- $R$ is the only proportion to satisfy $T(a, a, b, b)$ and $T(a, b, b, a)$.

- $P$ is the only proportion to satisfy $T(a, b, a, b)$ and $T(a, b, b, a)$.

- 6 proportions satisfying $T(a, b, a, b)$ including $A$ and $P$ but not $R$ and $I$.

- 6 proportions satisfying $T(a, b, b, a)$ including $P$ and $R$ but not $A$ and $I$.

- 6 proportions satisfying $T(a, a, b, b)$ including $A$ and $R$ but not $P$ and $I$.

Proof: The two last statements remain to be proved. See the main text for the other ones. Given a logical proportion $T(a, b, c, d)$ of the form $\left(I_{(a, b)}^{1} \equiv I_{(c, d)}^{2}\right) \wedge$ $\left(I_{(a, b)}^{3} \equiv I_{(c, d)}^{4}\right)$, reverse reflexivity enforces the two equivalences $I_{(a, b)}^{1} \equiv I_{(b, a)}^{2}$ and $I_{(a, b)}^{3} \equiv I_{(b, a)}^{4}$. Thus, the only choices for the equivalences are $a \wedge b \equiv c \wedge d$, $a \wedge \bar{b} \equiv \bar{c} \wedge d, \bar{a} \wedge b \equiv c \wedge \bar{d}, \bar{a} \wedge \bar{b} \equiv \bar{c} \wedge \bar{d}$ among the 16 possibilities. Since a logical proportion is defined from two distinct, non-ordered equivalences, there are $4 \times 3 / 2=6$ proportions satisfying $T(a, b, b, a)$. They are given in Table 16 . We recognize the proportions $R, P$, and the 4 conditional proportions $a|b:: d| c$, $b|a:: c| d, a|\bar{b}:: d| \bar{c}$, and $b|\bar{a}:: c| \bar{d}$.

Concerning sameness property, a similar reasoning applies leading to the fact that $I_{(a, a)}^{1} \equiv I_{(b, b)}^{2}$ and $I_{(a, a)}^{3} \equiv I_{(b, b)}^{4}$ where equivalence appears between conjunctions which do not share variables. This is only possible when $I_{(a, a)}^{1} \equiv$ $I_{(b, b)}^{2} \equiv \perp$ and $I_{(a, a)}^{3} \equiv I_{(b, b)}^{4} \equiv \perp$ leading to the pattern $a \wedge \bar{a}$. Thus we still have 6 candidates given in Table 17 where we recognize $A$ and $R$, and 4 degenerated proportions. Which completes the proof.

Proposition 6 There are only 12 proportions satisfying symmetry property. Apart 


$$
\begin{gathered}
a \bar{b} \equiv \bar{c} d \quad \bar{a} b \equiv c \bar{d}(R) \| a b \equiv c d \quad \bar{a} \bar{b} \equiv \bar{c} \bar{d}(P) \\
a b \equiv c d \quad \bar{a} b \equiv c \bar{d}\|a b \equiv c d \quad a \bar{b} \equiv \bar{c} d\| \bar{a} b \equiv c \bar{d} \quad \bar{a} \bar{b} \equiv \bar{c} \bar{d} \| a \bar{b} \equiv \bar{c} d \quad \bar{a} \bar{b} \equiv \bar{c} \bar{d}
\end{gathered}
$$

TABLE 16. The 6 proportions satisfying reverse reflexivity $T(a, b, b, a)$

$$
\begin{gathered}
a \bar{b} \equiv c \bar{d} \quad \bar{a} b \equiv \bar{c} d(A) \| a \bar{b} \equiv \bar{c} d \quad \bar{a} b \equiv c \bar{d}(R) \\
\bar{a} b \equiv \bar{c} d \quad \bar{a} b \equiv c \bar{d}\|\bar{a} b \equiv \bar{c} d \quad a \bar{b} \equiv \bar{c} d\| \bar{a} b \equiv c \bar{d} \quad a \bar{b} \equiv c \bar{d} \| a \bar{b} \equiv \bar{c} d \quad a \bar{b} \equiv c \bar{d}
\end{gathered}
$$

TABLE 17 . The 6 proportions satisfying sameness $T(a, a, b, b)$

from $P, A, I, R$ (homogeneous proportions), there are 4 conditional proportions and 4 hybrid proportions. (These proportions are shown in Table 14 in Annex A).

Proof: The symmetry property implies that

$$
T(a, b, c, d) \equiv T(c, d, a, b)
$$

and since both are logical proportions, Proposition 2 tells us that these 2 proportions should be identical up to a permutation of the 2 equalities. Let us denote $\sigma$ the permutation such that $\sigma(a)=c, \sigma(b)=d, \sigma(c)=a, \sigma(d)=b$. Back to our initial notation where $T(a, b, c, d) \triangleq I_{(a, b)} \equiv I_{(c, d)} \wedge I_{(a, b)}^{\prime} \equiv I_{(c, d)}^{\prime}, \sigma$ transforms an indicator for $(a, b)$ into an indicator for $(c, d)$ and vice-versa, the only options are:

- $\sigma\left(I_{(a, b)}\right)={ }_{I d} I_{(c, d)}$ and $\sigma\left(I_{(c, d)}\right)={ }_{I d} I_{(a, b)}$ (and similar for $I^{\prime}$ ): this means that the 2 sides of the equations are indicators of the same type i.e. $e q_{1}$ can only be $a \wedge b \equiv c \wedge d, \bar{a} \wedge b \equiv \bar{c} \wedge d, a \wedge \bar{b} \equiv c \wedge \bar{d}$ or $\bar{a} \wedge \bar{b} \equiv \bar{c} \wedge \bar{d}$. Since we have 4 choices for $e q_{1}$, it remains 3 choices for $e q_{2}$ which should be different from $e q_{1}$ to build up a proportion. Taking into account the fact that the order of the equations is not relevant, we get $4 \times 3 / 2=6$ such proportions.

- $\sigma\left(I_{(a, b)}\right)={ }_{I d} I_{(c, d)}^{\prime}$ and $\sigma\left(I_{(c, d)}\right)==_{I d} I_{(a, b)}^{\prime}$ (and similar by switching $I$ and $I^{\prime}$ ). The previous reasoning applies telling us that $I_{(a, b)}$ and $I_{(c, d)}^{\prime}$ should be of the same type leading to 4 possibilities. Then $I_{(a, b)}^{\prime}$ and $I_{(c, d)}$ should be of the same type leading to only 3 remaining possibilities. Then again $4 \times 3 / 2=6$ such proportions which achieves the proof.

Proposition 7. There are 16 proportions satisfying the central permutation property. Proof: It is sufficient to adapt the previous reasoning (Proposition 6) to this type of permutation. These proportions are given in Table 18.

$$
\begin{array}{cc||cc||cc||cc}
\bar{a} \bar{b} \equiv \bar{c} \bar{d} & \bar{a} b \equiv c \bar{d} & \bar{a} \bar{b} \equiv \bar{c} \bar{d} & a \bar{b} \equiv \bar{c} d & \bar{a} \bar{b} \equiv \bar{c} d & \bar{a} b \equiv c d & \bar{a} \bar{b} \equiv \bar{c} d & a \bar{b} \equiv \bar{c} \bar{d} \\
\bar{a} \bar{b} \equiv c \bar{d} & \bar{a} b \equiv \bar{c} \bar{d} & \bar{a} \bar{b} \equiv c \bar{d} & a b \equiv \bar{c} d & \bar{a} \bar{b} \equiv c d & \bar{a} b \equiv \bar{c} d & \bar{a} \bar{b} \equiv c d & a b \equiv \bar{c} \bar{d}(I) \\
\bar{a} b \equiv \bar{c} \bar{d} & a \bar{b} \equiv c d & \bar{a} b \equiv \bar{c} d & a \bar{b} \equiv c \bar{d}(A) & \bar{a} b \equiv c \bar{d} & a b \equiv c d & \bar{a} b \equiv c d & a b \equiv c \bar{d} \\
a \bar{b} \equiv \bar{c} \bar{d} & a b \equiv c \bar{d} & a \bar{b} \equiv \bar{c} d & a b \equiv c d & a \bar{b} \equiv c \bar{d} & a b \equiv \bar{c} \bar{d} & a \bar{b} \equiv c d & a b \equiv \bar{c} d
\end{array}
$$

TABLE 18 . The 16 proportions stable for central permutation 


\section{Proposition 9.}

- $A$ and $I$ are the only logical proportions satisfying symmetry and being stable for permutation $p 23$, i.e. the permutation of the means. The same result holds replacing $p 23$ by $p 14$ (permutation of extremes).

- $P$ and $I$ are the only logical proportions satisfying symmetry and being stable for permutation $p 12$. The same result holds replacing $p 12$ by $p 34$.

- There are 10 proportions including only 2 homogeneous ${ }^{11}$, namely $R$ and $I$, satisfying symmetry and being stable for permutation $p 24$. The same result holds replacing $p 13$ by $p 24$.

Proof: Regarding the first statement, it is sufficient for instance to compare Table 14 in Annex A, showing the proportions satisfying symmetry with Table 18 showing the proportions stable for central permutation: this gives us the result. Regarding the 2 other statements, checking the truth tables of the 12 proportions satisfying symmetry (shown in Table 14) gives the result.

Proposition 11. There are exactly 8 proportions satisfying the code independency property. Apart from the homogeneous proportions $P, A, I, R$, there are 4 hybrid proportions.

Proof: In fact, the code-independency property implies that:

$$
T(a, b, c, d) \equiv T(\bar{a}, \bar{b}, \bar{c}, \bar{d})
$$

Since both $T(a, b, c, d)$ and $T(\bar{a}, \bar{b}, \bar{c}, \bar{d})$ are logical proportions, Proposition 2 tells us that the 2 proportions should be identical up to a permutation of the 2 equalities. This exactly means that the second equivalence is obtained from the first one by negating all the variables. Since we have $4 \times 4$ equalities between indicators, we can build exactly $16 / 2=8$ proportions satisfying code independency property: each time we choose an equivalence, we use it and its negated form to build up a suitable proportion. Since $A, R, P, I$ are built this way, they satisfy code independency. These proportions are shown in Table 4.

Proposition 13. There are 54 transitive proportions: 2 homogeneous $A$ and $P, 4$ conditional proportions, namely $(a b \equiv c d) \wedge(a \bar{b} \equiv c \bar{d}) ;(a b \equiv c d) \wedge(\bar{a} b \equiv \bar{c} d) ;(a \bar{b} \equiv$ $c \bar{d}) \wedge(\bar{a} \bar{b} \equiv \bar{c} \bar{d}) ;(\bar{a} b \equiv \bar{c} d) \wedge(\bar{a} \bar{b} \equiv \bar{c} \bar{d})$, and the 48 degenerated proportions.

Proof: Let us consider first the degenerated proportions. They are built up with 3 distinct indicators only. Without loss of generality, we can consider a degenerated proportion of the form $T(a, b, c, d) \triangleq\left(I_{1}(a, b) \equiv I(c, d)\right) \wedge\left(I_{2}(a, b)=I(c, d)\right)$ where $I_{1}(a, b)$ distinct from $I_{2}(a, b)$. It appears that a degenerated proportion states the equivalence between 2 distinct indicators of the same pair (in that case $I_{1}(a, b)$ and $\left.I_{2}(a, b)\right)$. Thus, a valuation $\sigma$ validating such an equivalence between 2 distinct indicators of the same pair necessarily satisfies: $\sigma(I(c, d))=\sigma\left(I_{1}(a, b)\right)=$

\footnotetext{
${ }^{11}$ This corrects an erroneous statement in a previous paper [65], where we suggested that the 2 homogeneous proportions $R$ and $I$ were the only ones to satisfy these properties.
} 
$\sigma\left(I_{2}(a, b)\right)=0$ i.e. allocating 0 as truth value for all the indicators. Then a valuation $\sigma$ validating $T(a, b, c, d) \wedge T(c, d, e, f)$ satisfies $\sigma(I(c, d))=\sigma\left(I_{1}(a, b)\right)=$ $\sigma\left(I_{2}(a, b)\right)=\sigma(I(e, f))=\sigma\left(I_{1}(c, d)\right)=\sigma\left(I_{2}(c, d)\right)=0$. As a consequence, $\sigma$ validates $T(a, b, e, f)$ since $\sigma(I(e, f))=\sigma\left(I_{1}(a, b)\right)=\sigma\left(I_{2}(a, b)\right)=0$. And we are done for the degenerated proportions.

Thanks to Lemma 6, we can exclude the hybrid and semi-hybrid proportions from being transitive. Let us consider for instance the case of a hybrid proportion of the form: $T(a, b, c, d) \triangleq S_{(a, b)} \equiv D_{(c, d)} \wedge S_{(a, b)}^{\prime} \equiv D_{(c, d)}^{\prime}$. Let us consider $\sigma$ a valuation validating $T(a, b, c, d) \wedge T(c, d, e, f)$. We have 2 cases:

- either $\sigma\left(S_{(a, b)}\right)=1$ : then $\sigma\left(D_{(c, d)}\right)=1$ and thanks to Lemma $6, \sigma(c) \neq$ $\sigma(d)$. Again with Lemma $6, \sigma\left(S_{(c, d)}\right)=0$ which implies $\sigma\left(D_{(e, f)}\right)=0$ : then $\sigma\left(S_{(a, b)}\right) \neq \sigma\left(D_{(e, f)}\right)$ meaning that $T(a, b, e, f)$ does not hold.

- or $\sigma\left(S_{(a, b)}\right)=0$. If $\sigma\left(S_{(a, b)}^{\prime}\right)=1$, we are back to the previous case. If $\sigma\left(S_{(a, b)}^{\prime}\right)=0$, then both $\sigma\left(D_{(c, d)}\right)=0$ and $\sigma\left(D_{(c, d)}^{\prime}\right)=0$. Thanks to Lemma $6, \sigma(c)=\sigma(d)$ then at least one term between $\sigma\left(S_{(c, d)}\right)$ or $\sigma\left(S_{(c, d)}^{\prime}\right)$ is equal to 1 , thus preventing $\sigma\left(S_{(a, b)}\right)$ or $\sigma\left(S_{(a, b)}^{\prime}\right)$ to be equal to the corresponding term $\sigma\left(D_{(e, f)}\right)$ or $\sigma\left(D_{(e, f)}^{\prime}\right)$ : this means that $T(a, b, e, f)$ does not hold.

A similar reasoning does the job for semi-hybrid proportions. Regarding the 4 homogeneous proportions, a simple observation of the truth table shows that only $A$ and $P$ are transitive.

Finally, as the 16 conditional proportions express semantic equivalence (which is transitive) between conditional objects, it is clear that the 4 proportions $a \mid b::$ $c|d, b| a:: d|c, a| \bar{b}:: c|\bar{d}, b| \bar{a}:: d \mid \bar{c}$ are transitive (for instance $a|\bar{b}:: c| \bar{d} \wedge c|\bar{d}:: e| \bar{f}$ implies $a|\bar{b}:: e| \bar{f}$ due to the transitivity of the equivalence between conditional objects) but none of the remaining conditional proportions is transitive: for instance from $a|\bar{b}:: d| \bar{c} \wedge c|\bar{d}:: f| \bar{e}$, we cannot infer $a|\bar{b}:: f| \bar{e}$. Which achieves the proof.

Proposition 15. Among the 120 logical proportions, only 6 (among which the homogeneous $R$ and $I$ ) satisfy semi-mirroring, only 6 (among which the homogeneous $P$ and $I$ ) satisfy negation-compatibility, and only 6 (among which the homogeneous $A$ and I) satisfy exchange-mirroring.

Proof: Let us consider the substitution $\sigma$ such that $\sigma(c)=\bar{a}$ and $\sigma(d)=\bar{b}$. For semi-mirroring property to hold, the right hand side of the 2 equations should become equal to their left hand side when $\sigma$ is applied. And we have only 4 options which are $a \wedge b \equiv \bar{c} \wedge \bar{d}, \bar{a} \wedge \bar{b} \equiv c \wedge d, a \wedge \bar{b} \equiv \bar{c} \wedge d$ and $\bar{a} \wedge b \equiv c \wedge \bar{d}$. Since we need 2 of these equalities to build up a proportion, we have 6 choices only (among which we obviously have $I$ by combining the 2 first equalities and $R$ by combining the 2 last ones). The same reasoning applies to negation-compatibility and exchange-mirroring.

Proposition 17. There are 64 proportions for which the solution is always unique when it exists, and 56 proportions for which the equation $T(a, b, c, x)=1$ may have 
2 solutions for some entries. These 56 proportions divide into 8 conditional ones, 8 hybrid ones, 8 semi-hybrid ones, and 32 degenerated ones.

Proof: In that case, a simple (but fastidious) examination of the truth tables leads to the result.

Proposition 24. I is the unique Boolean formula (up to equivalence) satisfying these 2 sets of postulates.

Proof: Let us consider for instance the first set of postulates. For a logical proportion $T$ to satisfy non reflexivity, means that one valuation of the class $\{1111,0000$, $1010,0101\}$ is not valid. If this valuation is 1010 (or 0101), then central permutation and symmetry imply that 1100, 0011 and 0101 are not valid for $T$. Since $T$ has exactly 6 valid valuations, it means that among the remaining candidate valuations at least one has an odd number of 0. Let us assume 0111 (any other option would lead to the same result as a fact of symmetry) is valid: again symmetry and central permutation insure that the whole class $\{0111,1011,1110,1101\}$ is valid for $T$. And thanks to Lemma 5, this cannot be valid for a logical proportion.

Then, we have to consider that 1010,0101 are valid for $T$. Using central permutation, we get 1100 and 0011 are still valid for $T$. Since we have neither $\{0111,1011,1110,1101\}$ nor $\{1000,0100,0010,0001\}$, it remains to choose 2 valuations among $\{1111,0000,0110,1001\}$ excluding at least one among $\{1111,0000\}$ to complete the truth table of $T$. If we introduce 0110, we have to introduce 1001 to be consistent with symmetry and we get the truth table of $I$. If we remove one of the valuation 0110 or 1001, then we have to remove the other and the only remaining option for $T$ is to be valid for the valuations 1111, 0000, 1010, 0101, 1100, 0011. Which contradicts the initial assumption about $T$ (non reflexivity). Therefore, $I$ is the unique option. A similar reasoning does the job for the second set of postulates.

\section{ANNEX C: Conjectures}

Using a software described in [66], it becomes easier to investigate the universe of logical proportions. In particular, one can check the previous results, but we may also discover new properties, which may be more difficult to prove for some of them (otherwise than by tedious enumerations), and which may be even unexpected. In the following, we only mention conjectures discovered in this way that deal with the distribution of valuations among logical proportions, the study of properties similar to $T(a, a, a, a)$, but where some $a$ may be negated (e.g., $T(a, \bar{a}, a, \bar{a})$, or also $T(a, a, \bar{a}, a))$, and finally the study of the 20 hybrid proportions. But one may find many other results, such as for instance: Given a permutation, there are 16 proportions satisfying such a permutation except for $P_{1,2}$ and $P_{3,4}$ where there are 32 proportions.

Distribution of valuations among logical proportions 
A first series of conjectures pertains to the way the valuations that make logical proportions true (we have 16 distinct quaternary valuations, e.g. (0100)) are distributed among them:

- given any valuation $v$, there are exactly 45 proportions valid for $v$.

- given any pair of valuations $\left(v_{1}, v_{2}\right)$, there are exactly 15 proportions valid for $v_{1}$ and $v_{2}$.

- when we go for 3 randomly chosen valuations $\left(v_{1}, v_{2}, v_{3}\right)$, we can get 3 proportions or 6 proportions valid for these valuations. It means that whatever the triple of valuations, at least we have 3 valid proportions.

- on top of that, given 3 consecutive valuations, there are exactly 3 proportions valid for these valuations.

- when we go for 4 randomly chosen valuations, we can get 0 or 6 proportions.

- on top of that, given 4 consecutive valuations, there is no proportion valid for these valuations.

- when we go for 5 or 6 randomly chosen valuations, either we get 1 proportion or no proportion at all (obviously, there is no need to go for 7 , since there is no proportion having 7 valid valuations).

- given any pair of valuations $\left(v_{1}, v_{2}\right)$, there are exactly 30 proportions valid for $v_{1}$ and not valid for $v_{2}$.

\section{Homogeneity properties}

We have seen that the 15 proportions satisfying full identity, i.e., $T(a, a, a, a)$, are $A, R, P$, together with 8 conditional proportions, and 4 degenerated ones. See Proposition 3. But, we have not studied

- the 15 proportions satisfying $T(a, a, \bar{a}, \bar{a})$;

- the 15 proportions satisfying $T(a, \bar{a}, a, \bar{a})$;

- the 15 proportions satisfying $T(a, \bar{a}, \bar{a}, a)$.

Indeed,

- there are 15 proportions satisfying $T(a, a, \bar{a}, \bar{a})$; they are $A, R, I, 8$ conditional proportions, and 4 degenerated ones. Moreover, these 4 degenerated ones are the same as the ones for $T(a, a, a, a)$ (to reduce the size of the expressions, we omit the $\wedge$ symbol in the writing of the indicators, e.g., $\bar{a} \wedge b$ is abbreviated in $\bar{a} b)$ : $\bar{a} b \equiv \bar{c} d \wedge \bar{a} b \equiv c \bar{d}, \bar{a} b \equiv \bar{c} d \wedge a \bar{b} \equiv \bar{c} d, \bar{a} b \equiv c \bar{d} \wedge a \bar{b} \equiv c \bar{d}, a \bar{b} \equiv \bar{c} d \wedge a \bar{b} \equiv c \bar{d}$; the 8 conditional proportions associated with $T(a, a, \bar{a}, \bar{a})$ together with the 8 conditional ones associated with $T(a, a, a, a)$ make a partition of the 16 existing conditional proportions.

- there are 15 proportions satisfying $T(a, \bar{a}, a, \bar{a})$; they are $A, P, I, 8$ conditional proportions, and 4 degenerated ones.

- there are 15 proportions satisfying $T(a, \bar{a}, \bar{a}, a)$; they are $R, P, I, 8$ conditional proportions, and 4 degenerated ones.

Moreover, it can be noticed that the 4 degenerated ones are the same for $T(a, \bar{a}, \bar{a}, a)$ and for $T(a, \bar{a}, a, \bar{a}): \bar{a} \bar{b} \equiv \bar{c} \bar{d} \wedge \bar{a} \bar{b} \equiv c d, \bar{a} \bar{b} \equiv \bar{c} \bar{d} \wedge a b \equiv \bar{c} \bar{d}$,

$\bar{a} \bar{b} \equiv c d \wedge a b \equiv c d, a b \equiv \bar{c} \bar{d} \wedge a b \equiv c d$; the 8 conditional proportions associated 
with $T(a, \bar{a}, \bar{a}, a)$ together with the 8 conditional ones associated with $T(a, \bar{a}, a, \bar{a})$ make a partition of the 16 existing conditional proportions.

Besides, there are 6 proportions that satisfy:

- $T(a, a, a, a)$ and $T(a, a, \bar{a}, \bar{a}): A, R$, and 4 degenerated ones (already given)

- $T(a, a, a, a)$ and $T(a, \bar{a}, a, \bar{a}): A, P$, and 4 conditional ones: $\bar{a} \bar{b} \equiv \bar{c} \bar{d} \wedge \bar{a} b \equiv$ $\bar{c} d, \bar{a} \bar{b} \equiv \bar{c} \bar{d} \wedge a \bar{b} \equiv c \bar{d}, \bar{a} b \equiv \bar{c} d \wedge a b \equiv c d, a \bar{b} \equiv c \bar{d} \wedge a b \equiv c d ;$

- $T(a, a, a, a)$ and $T(a, \bar{a}, \bar{a}, a): P, R$, and 4 conditional ones: $\bar{a} \bar{b} \equiv \bar{c} \bar{d} \wedge \bar{a} b \equiv$ $c \bar{d}, \bar{a} \bar{b} \equiv \bar{c} \bar{d} \wedge a \bar{b} \equiv \bar{c} d, \bar{a} b \equiv c \bar{d} \wedge a b \equiv c d, a \bar{b} \equiv \bar{c} d \wedge a b \equiv c d$

- $T(a, a, \bar{a}, \bar{a})$ and $T(a, \bar{a}, a, \bar{a}): A, I$, and 4 conditional ones: $\bar{a} \bar{b} \equiv c d \wedge \bar{a} b \equiv \bar{c} d$, $\bar{a} \bar{b} \equiv c d \wedge a \bar{b} \equiv c \bar{d}, \bar{a} b \equiv \bar{c} d \wedge a b \equiv \bar{c} \bar{d}, a \bar{b} \equiv c \bar{d} \wedge a b \equiv \bar{c} \bar{d} ;$

- $T(a, a, \bar{a}, \bar{a})$ and $T(a, \bar{a}, \bar{a}, a): R, I$ and 4 conditional ones: $\bar{a} \bar{b} \equiv c d \wedge \bar{a} b \equiv c \bar{d}$, $\bar{a} \bar{b} \equiv c d \wedge a \bar{b} \equiv \bar{c} d, \bar{a} b \equiv c \bar{d} \wedge a b \equiv \bar{c} \bar{d}, a \bar{b} \equiv \bar{c} d \wedge a b \equiv \bar{c} \bar{d} ;$

- $T(a, \bar{a}, a, \bar{a})$ and $T(a, \bar{a}, \bar{a}, a): P, I$, and 4 degenerated ones (already given).

As can be seen, the 16 conditional proportions are partitioned into 4 groups of 4 proportions by the 4 conjunctions of two properties as indicated above. All these results provide counterparts to Proposition 3, and show regularities among logical proportions of the same kind.

If now we require that 3 among the 4 conditions hold

$-T(a, a, a, a)$

$-T(a, a, \bar{a}, \bar{a})$

$-T(a, \bar{a}, a, \bar{a})$

$-T(a, \bar{a}, \bar{a}, a)$

then it provides a unique characterization of each of the 4 proportions homogeneous proportions $A, R, P, I$, as it is obvious from their truth tables.

\section{Intruder properties}

But, one may also consider requirements, where the number of negations is odd rather than even as previously. Note that $T(a, a, a, \bar{a})$ is the same as $T(\bar{a}, \bar{a}, \bar{a}, a)$. We call these requirements intruder properties, since it expresses that one of the four items differ from the 3 others that are identical. They clearly contrast with the 4 homogeneity properties studied above, where the number of negations is even.

$-T(a, a, a, \bar{a})$

$-T(a, a, \bar{a}, a)$

$-T(a, \bar{a}, a, a)$

$-T(\bar{a}, a, a, a)$

Requiring 3 of them together defines a quaternary operator that is true for the 6 corresponding valuation patterns. But is it a logical proportion? The answer is yes. Here are the details:

- the one that satisfies $T(a, a, a, \bar{a}), T(a, a, \bar{a}, a)$, and $T(a, \bar{a}, a, a)$ is $\bar{a} b \equiv \bar{c} \bar{d} \wedge a \bar{b} \equiv c d$

and means "among $a, b, c, d$, there is an intruder (i.e., which is true, or false, alone) and which is not $a$ "; this proportion satisfies the permutation properties $p 23, p 24$, and $p 34$; 
- the one that satisfies $T(a, a, a, \bar{a}), T(a, a, \bar{a}, a)$, and $T(\bar{a}, a, a, a)$ is $a \bar{b} \equiv \bar{c} \bar{d} \wedge \bar{a} b \equiv c d$

and means "among $a, b, c, d$, there is an intruder (i.e., which is true, or false, alone) and which is not $b$ "; this proportion satisfies the permutation properties $p 13, p 14$, and $p 34$;

- the one that satisfies $T(a, a a \bar{a}), T(a, \bar{a}, a, a)$, and $T(\bar{a}, a, a, a)$ is

$\bar{a} \bar{b} \equiv \bar{c} d \wedge a b \equiv c \bar{d}$

and means "among $a, b, c, d$, there is an intruder (i.e., which is true, or false, alone) and which is not $c$ "; this proportion satisfies the permutation properties $p 12, p 14$, and $p 24$;

- the one that satisfies $T(a, a, \bar{a}, a), T(a, \bar{a}, a, a)$, and $T(\bar{a}, a, a, a)$ is

$\bar{a} \bar{b} \equiv c \bar{d} \wedge a b \equiv \bar{c} d$

and means "among $a, b, c, d$, there is an intruder (i.e., which is true, or false, alone) and which is not $d$ "; this proportion satisfies the permutation properties $p 12, p 13$, and $p 23$.

These 4 logical proportions are precisely the 4 non symmetrical hybrid proportions that satisfy code independency (see Proposition 12). They also have noticeable permutation properties; as can be seen, each permutation is satisfied by 2 proportions among the 4 .

As follows from their truth tables, these 4 logical proportions are true for valuation patterns that altogether gather the 8 patterns that make Klein's operator false, see Figure 8. Thus, these 4 logical proportions are in some sense, "opposed" to $A, R, P$, and $I$, which altogether gather the 8 other possible valuation patterns in their truth tables (restricted to those patterns that make them true). This is confirmed by the following properties. Among the whole set of logical proportions, each of $T(a, a, a, \bar{a}), T(a, a, \bar{a}, a), T(a, \bar{a}, a, a), T(\bar{a}, a, a, a)$ is incompatible with $T(a, a, a, a)$

each of $T(a, a, a, \bar{a}), T(a, a, \bar{a}, a), T(a, \bar{a}, a, a), T(\bar{a}, a, a, a)$ is incompatible with $T(a, a, \bar{a}, \bar{a})$

each of $T(a, a, a, \bar{a}), T(a, a, \bar{a}, a), T(a, \bar{a}, a, a), T(\bar{a}, a, a, a)$ is incompatible with $T(a, \bar{a}, a, \bar{a})$

each of $T(a, a, a, \bar{a}), T(a, a, \bar{a}, a), T(a, \bar{a}, a, a), T(\bar{a}, a, a, a)$ is incompatible with $T(a, \bar{a}, \bar{a}, a)$.

The 20 hybrid proportions: symmetry and permutations properties

While there are only 12 logical proportions that are symmetrical, there are 88 proportions that satisfy at least one permutation.

- the 4 hybrid proportions that are symmetrical (not to be confused with the 4 hybrid proportions satisfying code independency described above) have truth tables obeying the following specifications

- $T(a, \bar{a}, a, a), T(a, a, a, \bar{a})$, and (1 010 and 11111 ) : $\bar{a} \bar{b} \equiv \bar{c} d \wedge \bar{a} b \equiv \bar{c} \bar{d}$

- $T(a, \bar{a}, a, a), T(a, a, a, \bar{a})$, and ( 010101 and 00000$): a \bar{b} \equiv c d \wedge a b \equiv c \bar{d}$

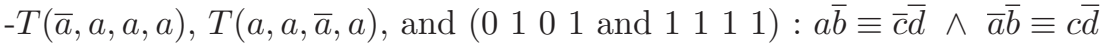


- $T(\bar{a}, a, a, a), T(a, a, \bar{a}, a)$, and (1 0100 and 00000$): \bar{a} b \equiv c d \wedge a b \equiv \bar{c} d$

They satisfy permutations $p 13$ and $p 24$.

- Regarding the 12 remaining hybrid proportions that do not satisfy neither symmetry nor code independency their truth tables obey to (with after, the permutations that they satisfy):

$* \bar{a} \bar{b} \equiv \bar{c} d \wedge \bar{a} b \equiv c d: T(\bar{a}, a, a, a), T(a, a, a, \bar{a})$, and the valuation patterns $(1$ 100 and 1010$)-p 23$

$* a \bar{b} \equiv \bar{c} \bar{d} \wedge a b \equiv c \bar{d}: T(\bar{a}, a, a, a), T(a, a, a, \bar{a})$, and the valuation patterns $(0$ 011 and 01101$)-p 23$

$* \bar{a} \bar{b} \equiv \bar{c} d \wedge a \bar{b} \equiv \bar{c} \bar{d}: T(\bar{a}, a, a, a), T(a, a, a, \bar{a})$, and the valuation patterns $(0$ 110 and 11111$)-p 23, p 14$

$* \bar{a} b \equiv c d \wedge a b \equiv c \bar{d}: T(\bar{a}, a, a, a), T(a, a, a, \bar{a})$, and the valuation patterns $(1$ 001 and 00000$)-p 23, p 14$

$* \bar{a} \bar{b} \equiv \bar{c} d \wedge a \bar{b} \equiv c d: T(a, \bar{a}, a, a), T(a, a, a, \bar{a})$, and the valuation patterns $(0$ 110 and 11100$)-p 13$

$* \bar{a} b \equiv \bar{c} \bar{d} \wedge a b \equiv c \bar{d}: T(a, \bar{a}, a, a), T(a, a, a, \bar{a})$, and the valuation patterns (1 0001 and 00011$)-p 13$

$* \bar{a} \bar{b} \equiv c \bar{d} \wedge \bar{a} b \equiv \bar{c} \bar{d}: T(a, \bar{a}, a, a), T(a, a, \bar{a}, a)$, and the valuation patterns (1 001 and 11111 ) - p23, p14

$* a \bar{b} \equiv c d \wedge a b \equiv \bar{c} d: T(a, \bar{a}, a, a), T(a, a, \bar{a}, a)$, and the valuation patterns ( 0 110 and 0000$)-p 23, p 14$

$* \bar{a} \bar{b} \equiv c \bar{d} \wedge \bar{a} b \equiv c d: T(\bar{a}, a, a, a), T(a, a, \bar{a}, a)$, and the valuation patterns (1 001 and 11100$)-p 24$

$* a \bar{b} \equiv \bar{c} \bar{d} \wedge a b \equiv \bar{c} d: T(\bar{a}, a, a, a), T(a, a, \bar{a}, a)$, and the valuation patterns $(0$ 110 and 00011$)-p 24$

$* \bar{a} \bar{b} \equiv c \bar{d} \wedge a \bar{b} \equiv c d: T(a, \bar{a}, a, a), T(a, a, \bar{a}, a)$, and the valuation patterns $(0$ 101 and 11100$)-p 14$

$* \bar{a} b \equiv \bar{c} \bar{d} \wedge a b \equiv \bar{c} d: T(a, \bar{a}, a, a), T(a, a, \bar{a}, a)$, and the valuation patterns (1 010 and 00011$)-p 14$

None of these 12 proportions satisfy permutations $p 12$ or $p 34$.

Henri Prade and Gilles Richard

Institut de Recherche en Informatique de Toulouse (IRIT)

Université Paul Sabatier, 118 route de Narbonne

31062 Toulouse Cedex 9, France

\{prade,richard\}@irit.fr 